\title{
Theoretical studies of singlet fission: searching for materials and exploring mechanisms
}

\author{
Achini Japahuge and Tao Zeng
}

\begin{abstract}
In this review article, a survey is given for theoretical studies in the subject of singlet fission. Singlet fission converts one singlet exciton to two triplet excitons. With the doubled number of excitons and the longer lifetime of the triplets, singlet fission provides an avenue to improve the photoelectric conversion efficiency in organic photovoltaic devices. It has been a subject of intense research in the past decade. Theoretical studies play an essential role in understanding singlet fission. In this paper, we review theoretical studies in singlet fission since 2006, the year when the research interest in this subject was reignited. Both electronic structure and dynamics studies are covered. Electronic structure studies provided guidelines for designing singlet fission chromophores and insights into the couplings between single- and multi-excitonic states. The latter gives us fundamental knowledge for engineering inter-chromophore conformation to enhance the fission efficiency. Dynamics studies reveal the importance of vibronic couplings in singlet fission.
\end{abstract}




\section{CONTENTS}

1. INTRODUCTION 4

2. THEORY 6

2.1. An Electronic Structure Description 6

2.2. The Roles of Vibronic Interaction 10

3. SEARCHING FOR SINGLET FISSION CHROMOPHORES 13

3.1. Alternant Hydrocarbons and Diradicals, the Two Limits 14

3.2. A Unified Diradical Character View of SF Chromophores 15

3.3. Small SF Chromophores 18

3.4. Chemically Modified Pentacenes 21

3.5. Summary of the Promising Theoretical Designs 22

3.6. A DFT-Based Protocol for Efficient Structural Search 23

4. EXPLORATION FOR SINGLET FISSION MECHANISMS THROUGH

ELECTRONIC STRUCTURE CALCULATIONS 24

4.1. Studies Using Oligomer Models 24

4.1.1. Transfer integrals and singlet fission, an early study 24

4.1.2. Quantum chemistry calculations of noncovalent oligomers of acenes and $\begin{array}{ll}\text { their derivatives } & 26\end{array}$

4.1.3. A transferable model 30

4.1.4. SF in covalent dimers of tetracene and pentacene 32

4.1.5. Using nonorthogonal configuration interaction to study SF 35

4.1.6. Using the norm of transition density matrix to study SF 37

4.1.7. Using the inexpensive PPP Hamiltonians to study SF 39

4.1.8. Solid state effects on charge-transfer energies 41

4.1.9. Construction of the five diabats with more extensive active space 43

4.1.10. Using vibronic coupling density to study SF 44

4.1.11. Using the Green's function approach to study SF 44

4.1.12. Including the charge-transfer states implicitly 47

4.2. Mapping SF Problems to Spin-Interaction Problems 48 
4.3. Model Developments for SF in Extended Systems 49

4.4. An Efficient Scheme to Locate SF-Favored Inter-Chromophore Conformations 52

\section{EXPLORATION FOR SINGLET FISSION MECHANISMS THROUGH}

DYNAMICS SIMULATIONS 53

5.1. Simulations with Phenomenological Dissipation and Decoherence 53

5.2. Development of System-Bath Models in SF 55

5.3. Including Vibrations in the System Hamiltonian 58

5.4. Application Studies $\quad 59$

5.4.1. Surface hopping simulations $\quad 59$

5.4.2. Symmetrical quasi-classical simulations 60

5.4.3. SF dynamics of perylenediimide $\quad 62$

5.4.4. Effects of surface polarity on SF dynamics 64

5.4.5. Multi-configuration time-dependent Hartree (MCTDH) simulations 65

5.4.6. A SF dynamics study for polyenes using electronic Hamiltonian 69

5.4.7. A second-order time-convolutionless quantum master equation study for $\begin{array}{ll}\text { pentacene } & 70\end{array}$

5.4.8. Diabats coupled to the same vibrational mode 70

5.4.9. The importance of a vibronic system Hamiltonian in tetracene SF 71

5.4.10. SF induced by Peierls coupling 71

5.4.11. J- or $H$-type coupling of SE $\quad 72$

5.5. Summary of Theoretical SF Time Scales 72

6. SEPARATION AND SPIN-DISENTANGLEMENT OF THE TRIPLET-PAIR 73

7. CONCLUSIONS AND OUTLOOK 75

8. ACKNOWLEDGEMENTS $\quad 78$

$\begin{array}{ll}\text { References } & 79\end{array}$ 


\section{INTRODUCTION}

In a conventional organic photovoltaic device, the absorption of a solar photon in the chromophore layer generates a singlet exciton. We label this excitonic state by $S_{1}$ for its character of the lowest $(1)$ singlet $(S)$ excited state of the chromophore molecule. Such an exciton can hop to the chromophore-acceptor interface, where the exciton undergoes charge separation to a hole and an electron. The holes and electrons are accumulated in electrodes and become free charge carriers. However, the $S_{1}$ exciton can undergo radiative and radiationless decays to the ground state (labelled as $S_{0}$ ), lowering the photoelectric conversion efficiency. All these steps are demonstrated in the upper panel of Figure 1, where the $S_{1}$ exciton is simplified to be localized in one chromophore molecule. In reality, the singlet exciton is delocalized and the range of delocalization is correlated with the bandwidth of the excitonic states, i.e., singlet exciton transfer integrals.

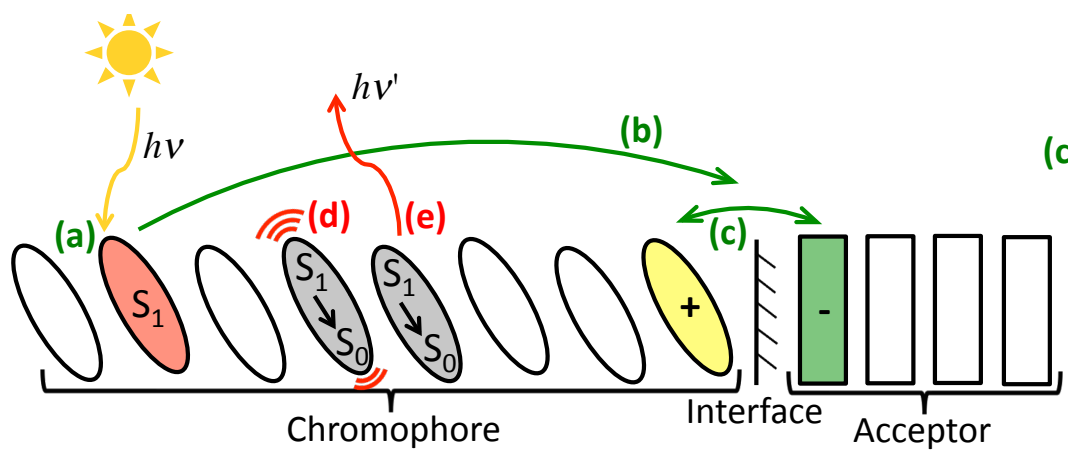

(c) at Interface
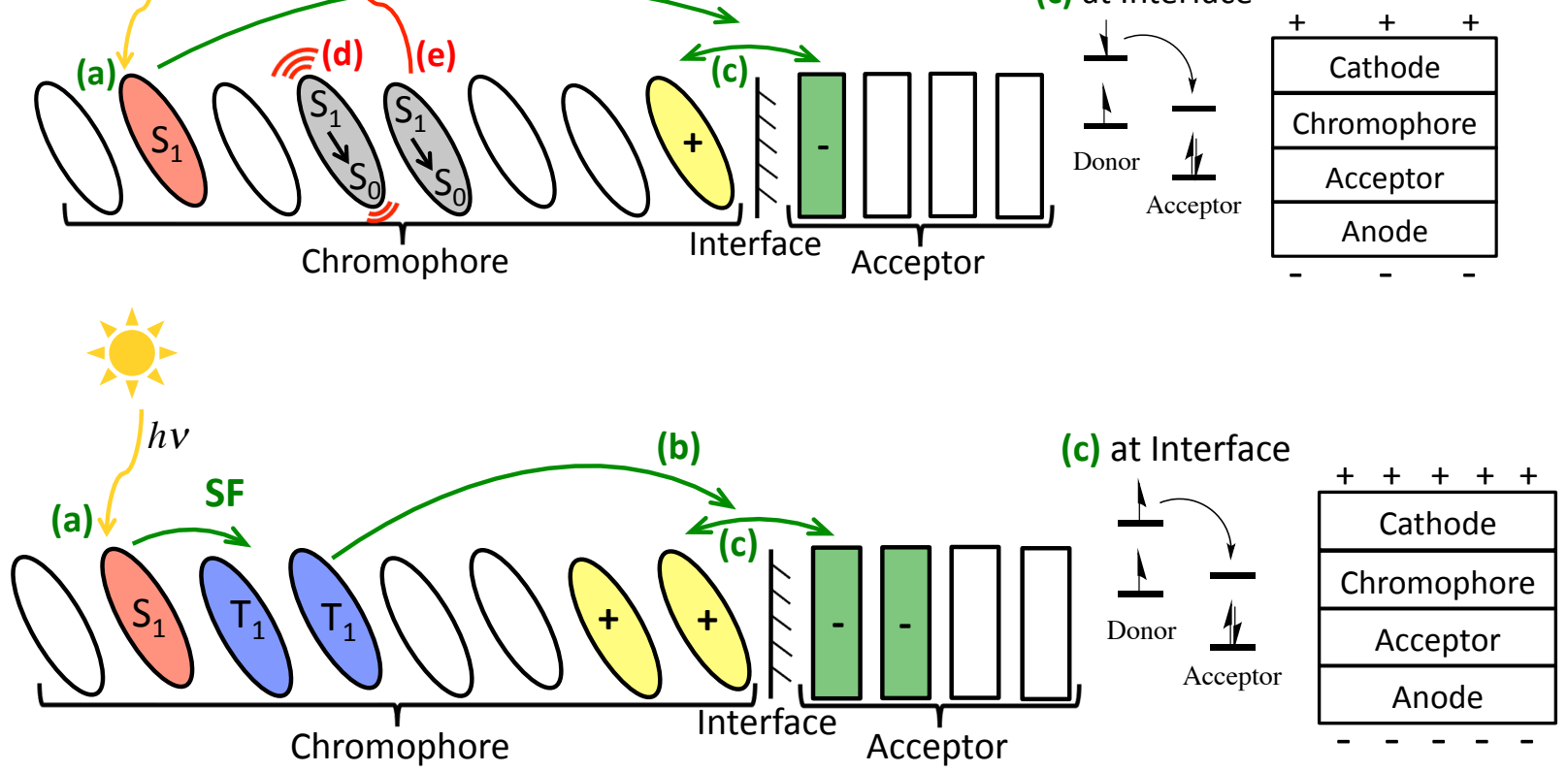

Figure 1. A comparison of photoelectric conversions without (upper panel) and with (lower panel) singlet fission. There are common steps in both panels: (a) photoexcitation, (b) exciton migration, and (c) charge separation between chromophore and acceptor. Steps (d) and (e) are radiationless and radiative decays of singlet exciton. 
Singlet fission (SF) is the process in which one $S_{1}$ exciton shares its energy with an adjacent chromophore that is in the ground state and converts to two triplet excitons on the two chromophores. ${ }^{1,2}$ The triplet excitons are labelled as $T_{1}$. SF starts with an internal conversion process (see below) that typically occurs on a sub-ps to ps time scale ${ }^{3-7}$ and outcompetes the $S_{1}$ radiative and radiationless decays, which usually occur on ns time scales. Molecules with ultrafast $S_{1}$-to- $S_{0}$ radiationless decay, e.g., azulene, ${ }^{8}$ are not appropriate SF chromophores. The number of excitons is doubled in SF. Furthermore, due to the spin selection rule, the $T_{1}$ excitons have a longer lifetime. The doubled number and longer lifetime enable more excitons to reach the interface and undergo charge separation, giving more electrons and holes at the electrodes. Therefore, SF provides an avenue to enhance photoelectric conversion efficiency. In their qualitative analysis, Hanna and Nozik showed that with SF, the conversion efficiency can surpass the $\sim 1 / 3$ Schockley-Queisser limit $^{9}$ of a single junction solar cell and reach $\sim 1 / 2 .{ }^{10}$ Indeed, organic photovoltaic (OPV) devices that exhibit $>100 \%$ quantum efficiency (electrons/photons ratio) have been fabricated, ${ }^{11-14}$ and the generation of more than 1 electron-hole pair by absorbing one photon is enabled by SF. A tandem architecture with a blue-absorbing SF donor and a red-absorbing acceptor is ideal for third generation solar cells..$^{1,10,15,16}$

Due to its importance in energy science, green chemistry, and organic photovoltaics, SF is a rapidly advancing field and has been the focus of a plethora of studies. Excellent review $\operatorname{articles}^{1,2,17}$ have been dedicated to this subject. Theoretical studies have been playing an important role in deepening our understanding of SF and designing novel SF materials and devices. However, up to date there has not been a review article to introduce and discuss these theoretical studies. This motivates us to write the present article, which is organized as follows. We first give a brief theoretical introduction of SF in Section 2. And then we review the theoretical studies dedicated to seek and design new SF chromophores in Section 3. In Sections 4 and 5, we review the electronic studies and dynamics studies dedicated to understand SF mechanisms, respectively. In Section 6, theoretical studies about the spin-disentanglement of the triplet-pair are introduced. Concluding remarks are given in Section. 7.

In this review, we focus on studies that employ theoretical calculations and simulations to obtain deeper understanding of SF, and studies that develop new methods to achieve this goal. Experimental studies with supporting computations are beyond the scope of this work. 
In one to two paragraphs we discuss essentials of a study, e.g., motivations, methods in use, systems under investigation, key conclusions, and/or methodology developments. All the reviewed studies were published in or after 2006. In that year, Hanna and Nozik published their work ${ }^{10}$ that rekindled extensive research interest in SF. Conclusions of the introduced studies may contradict, and these contradictions advance our knowledge of this complicated subject.

\section{THEORY}

\subsection{An Electronic Structure Description}

A SF process is schematically depicted in Figure 2, in which a dimer model of Chromophores A and B is used. Since the triplet-pair is residing on two chromophores, dimers are the smallest systems that can have SF. They can be covalently connected dimers or two adjacent molecules in a van der Waals solid. In each chromophore, only the highest occupied and the lowest unoccupied molecular orbitals (HOMO and LUMO) are considered. A SF process consists of two steps, the spin-conserved step and the spin-disentangled step. The spin-conserved step occurs within the singlet spin manifold and involves 5 electronic states: ${ }^{1,2}$ the single-excitonic states $e g$ and $g e$ that are generated by photoexcitation; $t t$, a singlet-coupled triplet-pair state; the charge transfer (CT) states $c a$ and $a c$. The vertical arrows (electronic spins) and horizontal bars (orbital levels) in Figure 2 indicate occupation schemes and characteristic spin alignments of the states. They shall not be understood as Slater determinants. Each state corresponds to a configuration state function that is spin-adapted and characterized by the corresponding occupation scheme. These states are

diabatic states (diabats) ${ }^{18}$ whose characters are invariant with respect to molecular geometry changes. Eigenstates of the electronic Hamiltonian, i.e., the adiabatic states (adiabats), are linear combinations of the diabats.

The off-diagonal Hamiltonian matrix elements of the diabats can be derived using the 


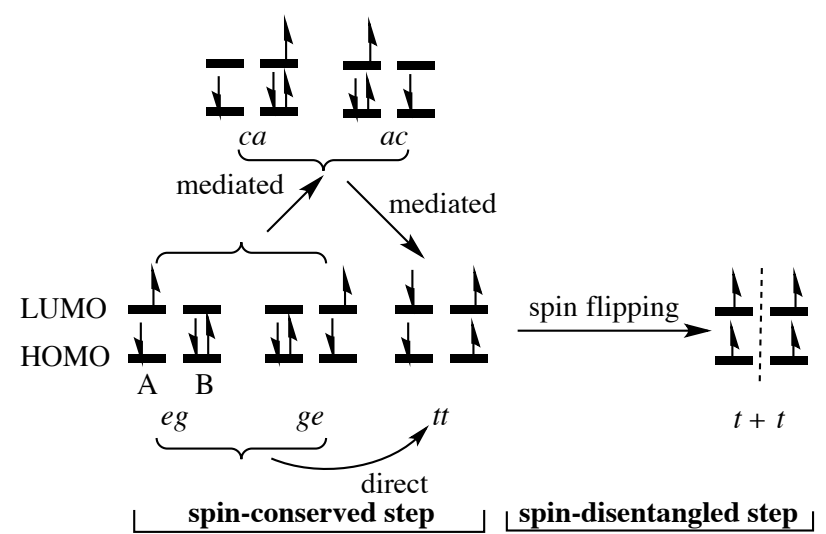

Figure 2. A schematic description of SF. The vertical arrows represent electronic spin-up and -down. In the state labels, $e$ stands for the HOMO-to-LUMO singlet exciton, $t$ the triplet exciton, $g$ the ground state, $c$ the cationic state, and $a$ the anionic state. A vertical dashed line is added to the $t+t$ state to emphasize that the two triplets are independent of each other.

Slater-Condon rules and they were given in Ref. 1:

$$
\begin{aligned}
H_{e g, t t} & =\sqrt{\frac{3}{2}}\left[\left\langle L_{A} L_{B} \mid H_{B} L_{A}\right\rangle-\left\langle H_{A} H_{B} \mid L_{B} H_{A}\right\rangle\right] \approx 0 ; \\
H_{e g, c a} & =t_{L_{A} L_{B}}+2\left\langle H_{A} L_{A} \mid L_{B} H_{A}\right\rangle-\left\langle H_{A} L_{A} \mid H_{A} L_{B}\right\rangle \approx t_{L_{A} L_{B}} ; \\
H_{e g, a c} & =-t_{H_{A} H_{B}}-2\left\langle H_{A} L_{A} \mid L_{A} H_{B}\right\rangle-\left\langle H_{A} L_{A} \mid H_{B} L_{A}\right\rangle \approx-t_{H_{A} H_{B}} ; \\
H_{c a, t t} & =\sqrt{\frac{3}{2}}\left[t_{L_{A} H_{B}}+\left\langle L_{A} L_{B} \mid H_{B} L_{B}\right\rangle-\left\langle L_{A} H_{A} \mid H_{B} H_{A}\right\rangle\right] \approx \sqrt{\frac{3}{2}} t_{L_{A} H_{B}} ; \\
H_{e g, g e} & =2\left\langle H_{A} L_{B} \mid L_{A} H_{B}\right\rangle-\left\langle H_{A} L_{B} \mid H_{B} L_{A}\right\rangle \approx 0 ; \\
H_{c a, a c} & =2\left\langle H_{A} L_{A} \mid L_{B} H_{B}\right\rangle-\left\langle H_{A} L_{A} \mid H_{B} L_{B}\right\rangle \approx 0 .
\end{aligned}
$$

Here, the symbol $\langle a b \mid c d\rangle$ denotes the two-electron (2e) integral $\int d r_{1} \int d r_{2} a\left(r_{1}\right) b\left(r_{2}\right) \frac{1}{r_{12}} c\left(r_{1}\right) d\left(r_{2}\right)$, $t_{a b}$ denotes the one-electron (1e) transfer integral (i.e., the matrix elements of the ground state Fock operator) between orbitals $a$ and $b$, and HOMO and LUMO have been abbreviated as $H$ and $L$. Because of their small magnitudes $(<10 \mathrm{meV})$ compared to the transfer integrals $(\sim 100 \mathrm{meV})$, one may set the 2e integrals to be zero and approximate the matrix elements by keeping the transfer integrals. The other off-diagonal matrix elements not included in Eq. 1 are obtained by swapping the chromophore indices $A$ and $B$. The small 2e integrals lead to generally small direct eg(ge)-tt couplings; SF relying solely on the direct couplings is unlikely to be efficient. Nevertheless, there may be exceptions. ${ }^{19}$

The CT states can mediate the eg(ge)-tt couplings. From the second order perturbation 
theory, one can derive the mediated $e g$ - $t$ (and similarly the $g e$ - $t$ t analogue) coupling $2,20,21$

$$
\begin{aligned}
V_{e g, t t} & =\frac{H_{e g, c a} H_{c a, t t}}{\Delta E_{c a}}+\frac{H_{e g, a c} H_{a c, t t}}{\Delta E_{a c}} \\
& \approx \sqrt{\frac{3}{2}}\left(\frac{t_{L_{A} L_{B}} t_{L_{A} H_{B}}}{\Delta E_{c a}}-\frac{t_{H_{A} H_{B}} t_{L_{B} H_{A}}}{\Delta E_{a c}}\right) .
\end{aligned}
$$

The 1e approximations in Eq. 1 have been used to obtain the second row of the equation. Depending on whether the CT states are treated as perturbing states for $e g$ or $t t, \Delta E_{c a}=$ $E(e g)-E(c a)$ or $E(t t)-E(c a)$. It can also be taken as their average. $\Delta E_{a c}$ assumes a similar formula. Due to the large magnitudes of the transfer integrals, the CT-mediated couplings are often more significant than the direct couplings. For instance, if we take a typical value $100 \mathrm{meV}$ for both $t_{L_{A} L_{B}}$ and $t_{L_{A} H_{B}}$ and a typical value $250 \mathrm{meV}$ for $\Delta E_{c a}$, $\frac{t_{L_{A} L_{B}} t_{L_{A} H_{B}}}{\Delta E_{c a}}=40 \mathrm{meV}$, an order of magnitude larger than the typical $1 \sim 5 \mathrm{meV}$ direct coupling. Therefore, SF usually proceeds through the CT-mediated pathway. One can sum up the direct and CT-mediated contributions to have the overall effective eg(ge)-tt couplings, which can be used in the Fermi's golden rule or the Marcus theory ${ }^{22}$ formula to estimate the $\mathrm{SF}$ rate.

The diagonal Hamiltonian matrix elements of the diabats, i.e., the diabatic energies, are also key parameters for SF. $E(e g)$ and $E(g e)$ shall be greater than $E(t t)$ to give an exoergic, thermodynamically favorable SF. Seeking chromophores that satisfy this condition is the main theme of Section 3. If the CT states lie higher in energy than $e g$ and $t t$, which is usually the case, the CT states act as virtual intermediate states and their populations are never substantial during the eg(ge)-to-tt conversion. This type of CT-mediated SF mechanism is called superexchange mechanism. ${ }^{20}$ If the CT states lie in energy between $e g$ and $t$, they are actual intermediate states and their populations have a noticeably rise-up due to the eg(ge)-to-ca(ac) conversion and then decay due to the $c a(a c)$-to- $t$ conversion. This type of CT-mediated SF mechanism is called sequential mechanism. The minus sign in the second row of Eq. 2, which arises from $H_{e g, a c}=-t_{H_{A} H_{B}}$ in Eq. 1, indicates a destructive interference between the two branches that pass through $c a$ and $a c$ in the CT-mediation. Alleviating the interference is a key to enhance the SF efficiency. We will see below that in some studies, the 5 diabats model is simplified to a 3 diabats model that include only eg, $t t$, and one of the CT states; the interference is absent. Usually those studies are dedicated to investigate the feasibility of the superexchange mechanism of SF; the absence of the interference does not affect the conclusions. 
The transfer and 2e integrals in Eqs. 1 and 2 involve inter-chromophore orbital overlaps. Therefore, the effective eg(ge)-tt couplings and the SF efficiency are highly sensitive to inter-chromophore configuration, ${ }^{1,2,23}$ which is "onerous to control" in solid materials. ${ }^{24}$ This difficulty motivates the idea of intramolecular singlet fission (iSF). The central idea is to covalently connect chromophore units and use steric hindrance to tune the inter-chromophore configuration. ${ }^{25}$ Recently, several iSF chromophores were designed and synthesized and they exhibited $>150 \%$ fission yield, the number of triplet excitons generated per photon. ${ }^{16,26-33}$

The second step shown in Figure 2 is the spin-disentangled step, in which the triplet-pair loses its spin-coupling as an overall singlet and becomes two independent triplet states with random orientations. This step is induced by spin-dependent Hamiltonians, e.g., the dipoledipole interaction between electronic spins and the electron-nuclear spin interaction. 1,34,35 These Hamiltonians do not commute with the total electronic spin operator. They mix the singlet-coupled triplet-pair state with the triplet- (if allowed by symmetry ${ }^{1}$ ) and quintetcoupled analogues. Along with thermalization, they randomize the orientations of the two triplets. These interactions are very weak, typically $<1 \mathrm{~cm}^{-1}$ in magnitude. This makes the disentanglement a slow process. A recent study that employed both transient absorption and time-resolved electron spin resonance spectroscopic techniques on 6,13-bis(triisopropylsilylethynyl)-pentacene (TIPS-pentacene) covalent dimers showed that it took hundreds of nanoseconds to lose the spin correlation of the triplet-pair. ${ }^{36}$ Similar experimental techniques were employed to study 6,13-bis(tri-isobutylsilylethynyl)-pentacene dimer with a non-conjugated linker. ${ }^{37}$ It took hundreds of nanoseconds for the singlet-coupled triplet-pair to convert to the quintet-coupled triplet-pair, which is consistent with the finding in Ref. 36. Furthermore, it took several microseconds to generate independent triplet excitons. The spin-disentanglement is so slow that the triplet states may have migrated apart yet their spins are still correlated. Because of the small magnitude of the spin-dependent interactions, (pseudo)degeneracy of the singlet-, triplet-, and quintet-coupled triplet-pair state is necessary for the spin-disentanglement. When the two triplets reside on adjacent molecules, they may be ferromagnetic or antiferromagnetic coupled and the three spin manifolds are of different energies, hindering the disentanglement. When they dissociate to be nonadjacent, the (anti)ferromagnetic coupling is ineffective and the degeneracy is achieved. Therefore, dissociation facilitates the spin-disentanglement.

We need to emphasize that Figure 2 gives a simplified description of SF. It is most relevant 
for SF across a dimer. It cannot describe the delocalization of the single-excitonic state beyond two monomers, neither the migration of the triplets. Also, the CT states may have different energies when the two chromophores are immersed in a solid environment. Another important difference is in the number of states (or density of states). In a dimer, there are two single-excitonic states and one multi-excitonic state. As the size of the oligomer grows, the number of single-excitonic states grows linearly, while that of multi-excitonic states grows quadratically. In combination with triplet migration, this faster growth of multi-excitonic states provides an entropy driving force for SF. As presented in the discussion below, the dimer model does deliver rich information of SF. However, conclusions based on this model shall not be straightforwardly generalized to explain SF in solids. In the text below, the symbols like $t t, e g$, etc. are only used to label dimeric diabats. States with similar singleand multi-excitonic characters in larger oligomers (trimer, tetramer, etc.) and solids are labelled by SE and ME.

\subsection{The Roles of Vibronic Interaction}

$\mathrm{SF}$ is an energy transfer process. Like in other energy transfer process, vibronic coupling plays a significant role in SF. The 5 diabats and their analogues in more extended systems can be viewed as forming the electronic system, which is coupled to a bath of vibrational degrees of freedom (phonons in solids). ${ }^{38}$ Thermodynamically speaking, the bath dissipates the energy released in the SE-to-ME conversion if it is an exoergic process. For an endoergic

SF process, which can be driven by entropy, ${ }^{7,39}$ the bath provides the needed energy.

The bath is commonly treated as a bunch of harmonic oscillators,

$$
\hat{H}_{B}=\sum_{i} \hbar \omega_{i}\left(\hat{p}_{i}^{2}+\hat{q}_{i}^{2}\right)
$$

and they are coupled to the system through modulating the electronic Hamiltonian matrix elements,

$$
H_{I J}=H_{I J}^{(0)}+\sum_{i} H_{I J}^{(1, i)} \hat{q}_{i}+\sum_{i j} H_{I J}^{(2, i j)} \hat{q}_{i} \hat{q}_{j}+\cdots
$$

Lower case indices $i$ and $j$ are used to label the oscillators and upper case $I$ and $J$ to label the diabats. $\hat{p}_{i}$ and $\hat{q}_{i}$ are dimensionless momentum and coordinate operators of the $i$-th oscillator. Usually, the expansion in Eq. 4 is truncated to only include the linear term. The 
couplings of the diagonal matrix elements are called the Holstein couplings, and those of the off-diagonal elements are called the Peierls couplings. ${ }^{40}$ The Holstein couplings bring about different potential energy surfaces (PESs) for different diabats. Figure 3(a) demonstrates the effects of Holstein couplings in an ideal SF process. The Franck-Condon excitation $(h \nu)$ vertically shifts the ground state $(\mathrm{GS})$ vibrational wave function to the SE potential energy surface (simplified as a curve in the figure, in an abstract vibrational coordinate $q$ ). The ground state structure corresponds to an interaction region where the SE and ME energies are close and the SE-to-ME transition induced by the matrix element $H_{S E, M E}$ is efficient, resulting in a vibrational wave packet on the ME PES. $H_{S E, M E}$ is assumed to be fixed in this model. The back-and-forth population conversion between SE and ME continues, which is the Rabi oscillation. Meanwhile, the wave packets on the different PESs evolve following the respective potentials and migrate away from the vertical interaction region. All wave packets in the figure are simplified as gaussians. Given that the ME PES has a minimum lower than the SE PES, the ME wave packet samples structures with larger SE-ME energy gaps than the SE wave packet. Consequently, the ME-to-SE conversion is slower than the SE-to-ME conversion. The efficiency of an $H_{S E, M E}$-driven conversion is reflected by the transparency of the relevant arrow in Figure 3(a): the more transparent, the slower. These unbalanced conversion rates remove the Rabi oscillation and result in an overall unidirectional SE-toME conversion. The far distance between minima in PESs lead to small overlap between the SE and ME wave packets, i.e., a decoherence between the two states. The decoherence provides another way to understand why vibronic coupling destroys the Rabi oscillation. ${ }^{41}$ If the Franck-Condon region is far from the interaction region, the Holstein coupling also brings the SE wave packet to the interaction region.

Typical evolutions of populations in SF from dynamics simulations with and without considering vibronic interaction are compared in Figures 3(b) and (c). As elucidated above, the former exhibits a unidirectional SE-to-ME population conversion while the latter shows a Rabi oscillation. Evidently, there is no SF if there are no vibronic couplings. The Holstein couplings usually involve high frequency intra-chromophore vibrations because the monomeric $S_{1}$ and $T_{1}$ excitations often involve $\pi$-to- $\pi^{*}$ transitions and are accompanied with changes in bond lengths. Inter-chromophore vibrations are of low frequencies and may contribute to the Holstein couplings of CT states, as the Coulomb interaction between the charged chromophores is dependent on their distance and conformation. If the CT states 

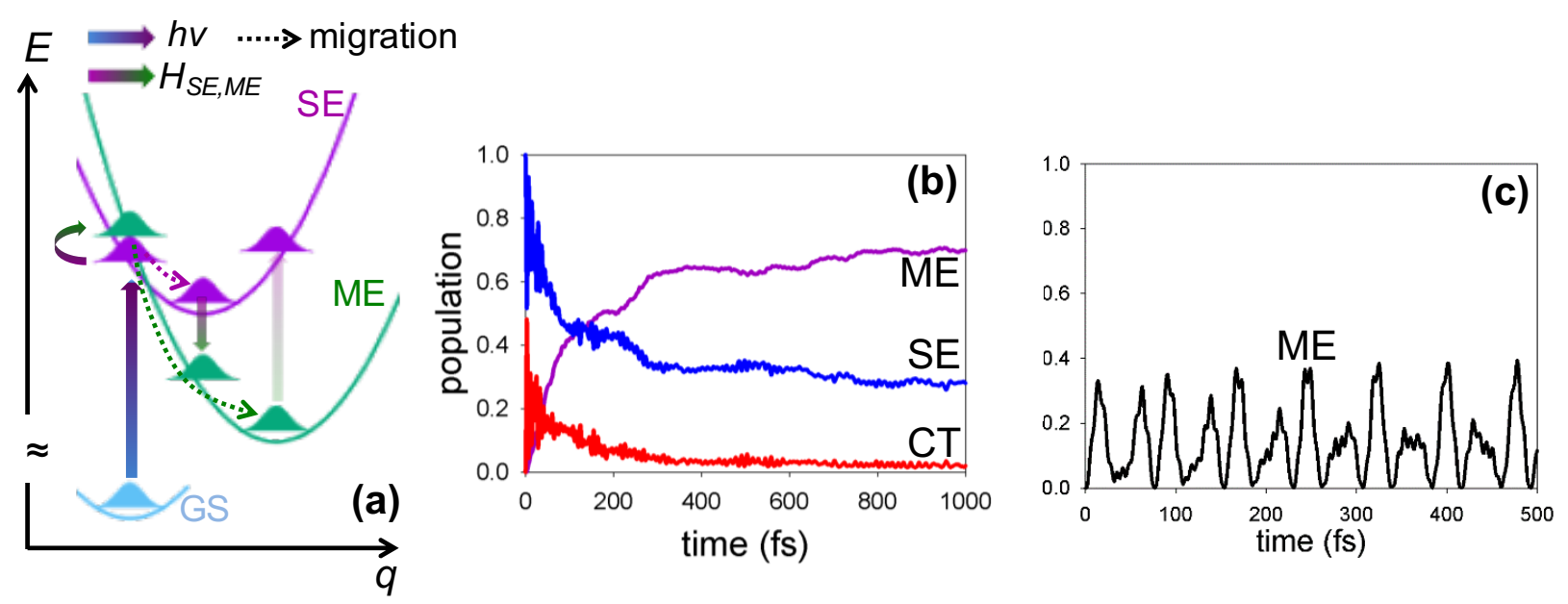

Figure 3. (a) Vibrational wave packets on different potential energy surfaces and their transitions to different states in SF; evolutions of diabatic populations in a SF process from dynamics simulations that consider (b) vibronic interaction and (c) only the electronic Hamiltonian. In Panel (c) only the ME state population is plotted to show the Rabi oscillation. Panels (b) and (c) are adapted with permission from Figures S3 and S5 in Ref. 41; Copyright 2015, American Physics Society.

are viewed as the virtual intermediates, their Holstein couplings essentially become Peierls couplings of the SE-ME effective couplings (see the next paragraph for an example).

The Peierls couplings modulate the off-diagonal $H_{I J}$. They are especially important when the effective SE-ME couplings are zero at equilibrium geometry. This can happen for a symmetry reason. ${ }^{41,42}$ Under this circumstance, the Peierls couplings lead to fluctuations of the SE-ME couplings and open a channel for the fission. Since all matrix elements in Eq. 1 involve inter-chromophore orbital overlaps, the Peierls couplings are more sensitive to inter-chromophore vibrations of low frequencies. The Holstein couplings can have a similar effect. For instance, if the two terms in Eq. 2 cancel each other at the equilibrium geometry, the fluctuations of $\Delta E_{c a}$ and $\Delta E_{a c}$ induced by the Holstein couplings can alleviate this destructive interference and turn on the fission. Given its indispensability, vibronic coupling has been the subject of more and more recent SF studies, as discussed in Section 5 . 


\section{SEARCHING FOR SINGLET FISSION CHROMOPHORES}

SF was first observed in crystalline anthracene (1) in $1965^{43}$ and its studies were immediately extended to tetracene $(\mathbf{2})$ and pentacene $(\mathbf{3}) .{ }^{44-49}$ A few materials are known to undergo SF efficiently. They include tetracene, pentacene, some of their derivatives, ${ }^{4,6,7,11,12,50-62}$ perylenediimide, ${ }^{63}$ as well as several conjugated polymers. ${ }^{26,64-68}$ An emerging class of SF chromophores are non-polycyclic thienoquinoid compounds. ${ }^{69}$ This highly limited arsenal of SF chromophores obstructs the application of SF in OPV. This difficulty motivated a series of studies in understanding the intrinsic characters of the chromophores and searching for new chromophores. The key energetic requirements for SF chromophores are

$$
\begin{aligned}
& E\left(S_{1}\right) \geq 2 E\left(T_{1}\right) ; \\
& E\left(T_{2}\right) \geq 2 E\left(T_{1}\right) ; \\
& E\left(Q_{1}\right) \geq 2 E\left(T_{1}\right) .
\end{aligned}
$$

The first condition guarantees that SF is an exoergic, thermodynamically favorable process. All three conditions ensure the unfavorableness of the reversed triplet-triplet fusion, which may result in a singlet, a triplet, or a quintet state.
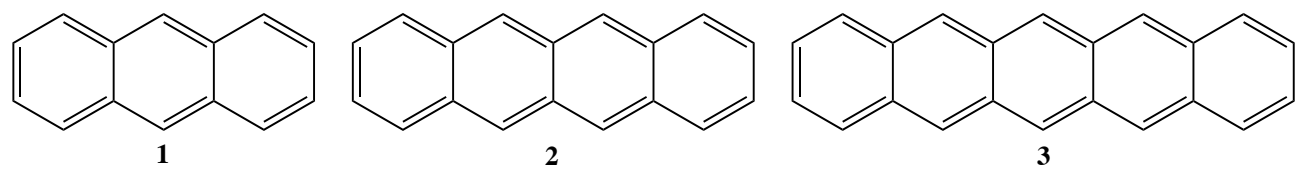

$T_{1}$ typically involves exciting an electron from HOMO to LUMO and flipping its spin, while $Q_{1}$ involves the second excitation and spin-flipping from $\mathrm{HOMO}-1$ to $\mathrm{LUMO}+1$, which costs more energy than the first excitaiton. Therefore, Eq. 7 is usually satisfied and can be ignored. Two $T_{1}$ excitons are unlikely to recombine to one molecule in $T_{1}$ and one molecule in $S_{0}$ due to the substantial energy release by $E\left(T_{1}\right)$ (i.e., the energy gap law). Instead, they may recombine to form one molecule in $T_{2}$ and the other in $S_{0} ; E\left(T_{2}\right)$ might be close to $2 E\left(T_{1}\right)$. Eqs. 5 and 6 guide the search for SF-capable chromophores. Since $S_{1}$ usually involves HOMO-to-LUMO excitation (Class I chromophores defined in Ref. 1) while $T_{2}$ involves excitation with a larger orbital energy gap, e.g., HOMO-to-LUMO+1, HOMO-1to-LUMO, etc., $E\left(T_{2}\right)$ is likely to be larger than $E\left(S_{1}\right)$. Therefore, we can by and large focus on Eq. 5. However, $E\left(T_{2}\right)$ shall be always examined in designing SF chromophores. In addition to Eq. 6, $E\left(T_{2}\right)>E\left(S_{1}\right)$ is desired. ${ }^{1,2}$ This inequality ensures a thermodynamically 
unfavored $S_{1}$-to- $T_{2}$ intersystem crossing (ISC), which is followed by the efficient $T_{2}$-to- $T_{1}$ decay (Kasha's rule ${ }^{70}$ ); only one $T_{1}$ exciton results. Although $E\left(T_{2}\right)>E\left(S_{1}\right)$ is desired, it is not as a strong condition as Eqs. 5 and 6 . The states involve $\pi$-to- $\pi$ excitations. According to El-Sayed's rule, ${ }^{71,72}$ ISC between $\pi$ excited states is usually slow, although it may be thermodynamically favorable. The energies in Eqs. 5 to 7 shall be those of the optimized structures of the respective states, because vibrational relaxation is competitively fast compared to the fission and fusion. Other than the energy relations, $E\left(T_{1}\right)$ close to $1 \mathrm{eV}$ is desired. This gap maximizes the efficiency in energy conversion. ${ }^{1}$

\subsection{Alternant Hydrocarbons and Diradicals, the Two Limits}

The structural search has been concentrated on $\pi$-conjugated systems because of their good absorption in an extensive range of photon frequencies. The first systematic search for

chromophores that satisfy Eq. 5 was performed by Paci et al. ${ }^{73}$ They proposed two classes of parental structures that can be modified to meet the requirement: the closed-shell alternant hydrocarbons in the left end of Figure 4 and the open-shell diradicals in the right end. When both $S_{1}$ and $T_{1}$ involve one electron HOMO-to-LUMO excitation, their energies differ by twice the exchange integral between the two orbitals $\left(2 K_{h l}\right)$. For alternant hydrocarbons, this gap is largely invariant. ${ }^{1}$ Therefore, by reducing $E\left(S_{1}\right)$ (or likewise the HOMO-LUMO gap), we will eventually reach the structures that satisfy $E\left(S_{1}\right) \geq 2 E\left(T_{1}\right)$. Indeed, as the length of acene increases and the HOMO-LUMO gap decreases, ${ }^{74}$ all acenes longer than and including tetracene satisfy this condition. In the other end, diradicals have triplet ground states, while the lowest singlet $\left(S_{0}\right)$ is higher by twice the exchange integral between the two singly occupied orbitals $A$ and $B\left(2 K_{A B}\right) . E\left(S_{1}\right) \geq 2 E\left(T_{1}\right)$ is automatically satisfied. However, diradicals are in general unstable. By increasing the overlap between orbitals $A$ and $B$, and hence the HOMO-LUMO gap (HOMO: the bonding orbital between $A$ and $B$; LUMO: antibonding), the resultant more stable diradicaloid has $S_{0}$ as the ground state and $E\left(T_{1}\right)$ increases. When $E\left(T_{1}\right)$ increases to near but still less than $\frac{1}{2} E\left(S_{1}\right)$, a promising $\mathrm{SF}$ chromophore is reached. The two classes of structures are not mutually exclusive, as the HOMO-LUMO gap and diradical character are correlated. ${ }^{74-76}$

Paci et al. used the efficient Pariser-Parr-Pople (PPP) method ${ }^{77-79}$ to calculate geometrically relaxed $E\left(S_{1}\right) \mathrm{s}, E\left(T_{1}\right) \mathrm{s}$, and $E\left(T_{2}\right) \mathrm{s}$ for more than 60 structures of the two classes. 


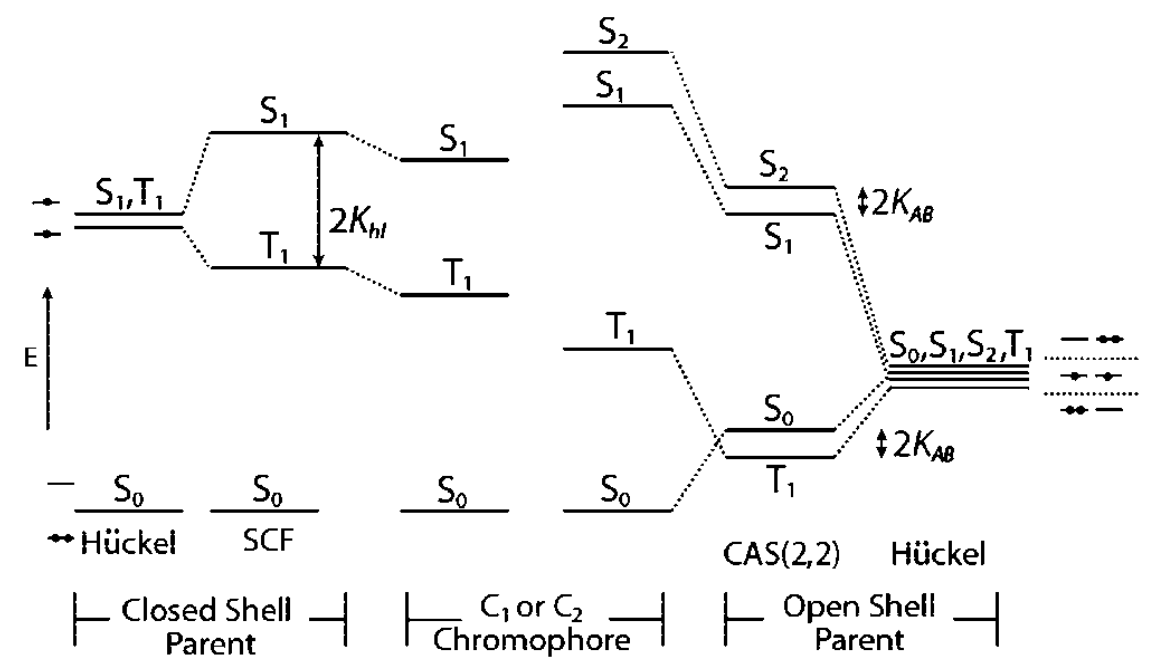

Figure 4. Characteristic energy levels of the closed- and open-shell parental structures (at the left and right ends), and their changes when structures are perturbed towards singlet fission chromophores (in the middle). This figure is taken with permission from Ref. 1; Copyright 2010, American Chemical Society.

Several $o$-and $p$-xylylene derivatives were predicted to be promising chromophores. Among them, 1,3-diphenylisobenzofuran (4, DPB) has been shown to undergo $\mathrm{SF}{ }^{80-82} \mathrm{DPB}$ is the first theoretically designed chromophore that turned out to undergo SF. It has attracted lots of research interest. ${ }^{80-84}$
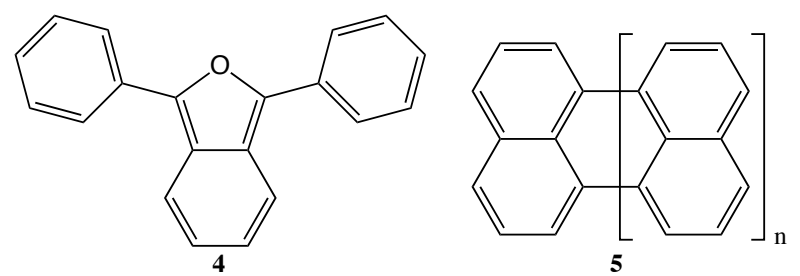

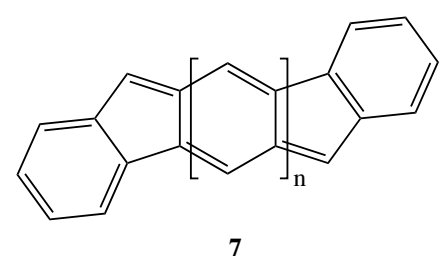

\subsection{A Unified Diradical Character View of SF Chromophores}

Considering the close connection between the two classes of parental structures for SF chromophores, Minami and Nakano developed a unified view on searching for SF chromophores based on multiradical character. ${ }^{85}$ They used a simple linear $\mathrm{H}_{4}$ model of $D_{\infty h}$ symmetry to investigate the relation between excitation energies and diradical/tetraradical character. Such a model with 4 orbitals and 4 electrons (4o4e) allows to simulate the $T_{2}$ 
state of a chromophore. By varying the three $\mathrm{H}-\mathrm{H}$ internuclear distances, they freely tuned the ground state character of the $\mathrm{H}_{4}$ model from a perfect closed-shell $(\mathrm{H}-\mathrm{H} \cdots \mathrm{H}-\mathrm{H})$, to a diradical $(\mathrm{H} \cdots \mathrm{H}-\mathrm{H} \cdots \mathrm{H})$, and to a tetraradical $(\mathrm{H} \cdots \mathrm{H} \cdots \mathrm{H} \cdots \mathrm{H}) . E\left(S_{1}\right)-2 E\left(T_{1}\right)$ and $E\left(T_{2}\right)-2 E\left(T_{1}\right)$ were calculated for each $\mathrm{H}_{4}$ conformation and plotted against the occupation numbers $\left(y_{0}\right.$ and $\left.y_{1}\right)$ of the lowest unoccupied natural orbital (LUNO) and the second lowest one $(\mathrm{LUNO}+1)$, which respectively indicate diradical and tetraradical characters of a molecule. Through inspecting the variations of the two differential energies with respect to $y_{0}$ and $y_{1}$, the authors proposed the following guidelines for the chromophore search: (1) the chromophore should have considerable but not too much diradical character in order to satisfy $E\left(S_{1}\right)-2 E\left(T_{1}\right)$ greater than and close to 0 , so that the energy efficiency in SF is high; (2) the tetraradical character of a chromophore should be minute to satisfy $E\left(T_{2}\right)-2 E\left(T_{1}\right)>0$. Several known SF chromophores fall in this ideal region of diradical/tetraradical character. These fundamental guidelines significantly facilitated the chromophore search in the following years. Immediately following this study, Minami et al. calculated $E\left(S_{1}\right), E\left(T_{1}\right)$, and $E\left(T_{2}\right)$ for a series of oligorylenes (5), whose diradical character increases with their length $(n) .{ }^{86}$ They identified terrylene $(n=2)$ and quaterrylene $(n=3)$ as promising SF chromophores.

Because of the correlation between reduced aromaticity and increased diradical character, ${ }^{87,88}$ Ito et al. investigated the feasibility of SF in two series of anti-aromatic polycyclic hydrocarbons with $4 n \pi$ electrons. ${ }^{89}$ The hydrocarbons are based on pentalene and dicyclopenta-fused acenes core structures (6 and 7 ). The pentalene-based structures bare two acenes on the two sides, each of which has increasing diradical character as their length increases. Therefore, the pentalene-based structures have both diradical and tetraradical characters increase as $n$ increases. On the other hand, the dicyclopenta-fused acenes only exhibit larger diradical character along with the increasing $n$, with the two unpaired electrons localized on the 5-membered rings. The calculated excitation energies of the dicyclopentafused acenes with $n \geq 2$ satisfy the two energy criteria, while the pentalene-based structures failed to satisfy Eq. 6 up to $n=6$. The $n=2$ structure was predicted to be the best chromophores along the dicyclopenta-fused acene series due to its lowest energy loss in SF.

Using a two-site model in Figure 5, Minami et al. derived a semi-quantitative relation 
between $2 E\left(T_{1}\right)-E\left(S_{1}\right)$ and $y_{0} \cdot{ }^{90}$

$$
f \equiv \frac{2 E\left(T_{1}\right)-E\left(S_{1}\right)}{U}=-\frac{3}{2}+\frac{1}{2 \sqrt{1-\left(1-y_{0}\right)^{2}}}-\frac{2 K_{a b}}{U} .
$$

The model consisted of 2 electrons in HOMO ( $g$ for gerade) and LUMO ( $u$ for ungerade), or equivalent in the two localized natural orbitals (LNO $a$ and $b$ ). $U=E\left(S_{1}\right)-E\left(T_{1}\right)=2 K_{g u}$, twice the exchange integral between HOMO and LUMO, and $K_{a b}$ is the exchange integral between orbitals $a$ and $b$. Eq. 8 clearly shows that $y_{0}$ is not the only variable that determines the sign of $2 E\left(T_{1}\right)-E\left(S_{1}\right) . K_{a b}$ is the other factor. With the $2 \frac{K_{a b}}{U} \approx 0$ approximation, the authors obtained the optimal range of $y_{0}$ that gives negative $f$ and yet $f \approx 0: 0<y_{0} \leq 0.06$. This range of $y_{0}$ is however too small compared to the optimal $y_{0}$ values obtained in Refs. 86 and 89. The deviation arises from the approximation of the two-site model. Because $y_{0}$ can be estimated using observed low-lying excitation energies, ${ }^{91}$ this semi-quantitative $f\left(y_{0}\right)$ functional allows for a quick screening of possible SF chromophores through inspecting their tabulated excitation energies.

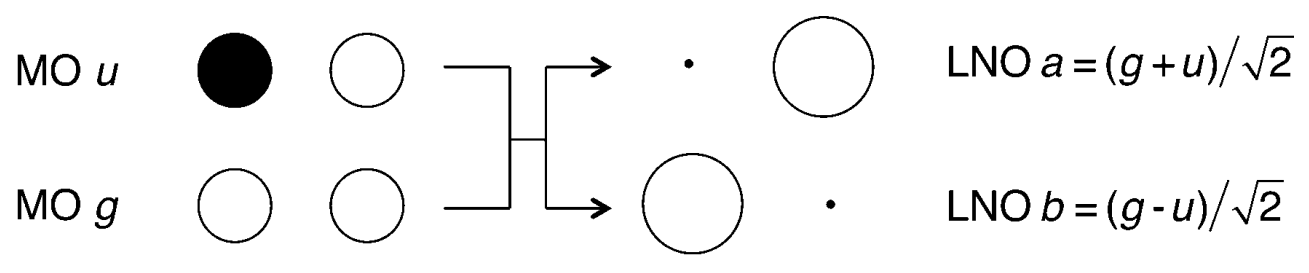

Figure 5. The delocalized molecular orbitals and localized natural orbitals used in the 2o2e model in Ref. 90. This figure is taken with permission from Ref. 90; Copyright 2013, American Chemical Society.

The diradical character can be enhanced by increasing aromaticity of a fragment of a molecule. Ito and Nakano investigated a series of heteroacene models based on core structures of $m$-xylylene $(N m-i, 8) .{ }^{92}$ The authors tuned the aromaticity of the central rings by N-substitution (changing $m$ ) and modified the $\pi$-conjugation extension (changing $i$ ). The increases of the aromaticity and conjugation length of the central rings strengthen the diradical character with the resonance structure shown in $\mathbf{8}$, and the model molecules start to satisfy Eq. 5. N2 -4 and $N 0-3$ were identified as ideal SF chromophores: they satisfy the energy criteria and have $E\left(T_{1}\right)$ close to $1 \mathrm{eV}$.

One way to design SF chromophores is to start with a structure with too much diradical character and with $2 E\left(T_{1}\right)<E\left(S_{1}\right)$, and then introduce substitutions to mitigate the 

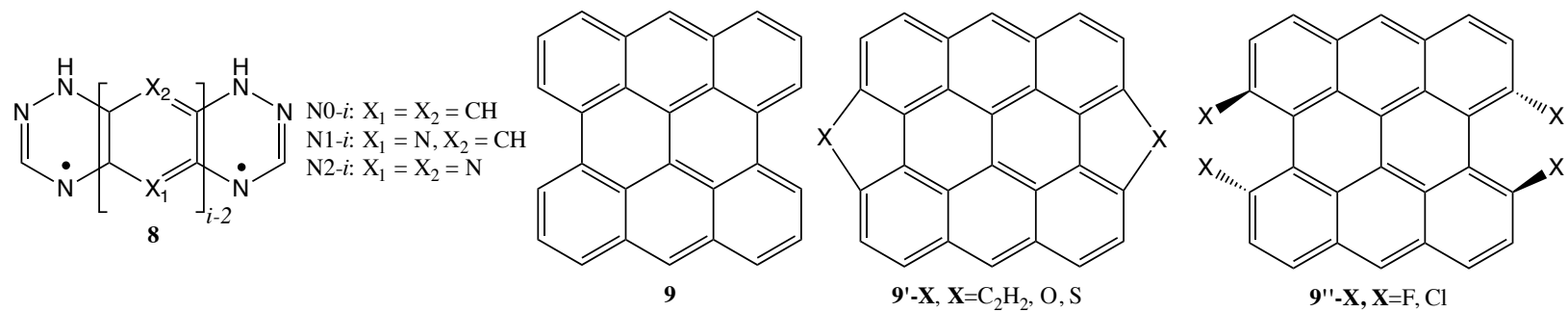

diradical character to make $2 E\left(T_{1}\right)$ closer to $E\left(S_{1}\right)$. Following this route, Ito et al. designed SF chromophores with bisanthene $(\mathbf{9})$ as a starting structure ${ }^{93}$ Bisanthene possesses too significant diradical character. Its calculated $E\left(S_{1}\right)$ and $E\left(T_{1}\right)$ were 2.393 and $0.939 \mathrm{eV}$. $2 E\left(T_{1}\right)-E\left(S_{1}\right)=-0.515$ indicates a substantial energy loss in SF. Ito et al. reduced the diradical character by making $\mathrm{O}-, \mathrm{S}-$, and $\mathrm{C}_{2} \mathrm{H}_{2}$-substitutions as in $\mathbf{9}$ '- $\mathbf{X}$. The substitutions introduce two extra sextet rings to the non-diradical resonance structure and hence reduce the diradical character. The authors also substituted bisanthene with bulky halogen atoms and methyls as in $\mathbf{9 " - X}$ to twist the structure to non-planar. The non-planarity reduces the overlaps between the frontier orbitals of the two anthracene units and enlarges the HOMOLUMO gap, reducing the diradical character. 9'-O and 9"'-F were predicted to be promising chromophores, with $2 E\left(T_{1}\right)-E\left(S_{1}\right) \approx 0$.

\subsection{Small SF Chromophores}

Conventional SF chromophores like tetracene, pentacene, and their derivatives are fairly large in size. Akdag et al. pioneered in designing small SF chromophores. ${ }^{94}$ Small chromophores are desired because: (1) they can give high exciton density, which eventually facilitates the development of mini OPV devices; (2) although they may not be stable, they serve as core structures for more kinetically persistent derivatives; (3) they are convenient models for theoreticians to investigate $\mathrm{SF}$, like $\mathrm{CH}_{2}$ for carbene chemistry research. The authors proposed 5 monocyclic structures. They are 5- or 6-membered aromatic rings with endocyclic electron-donors ( $\mathrm{sp}^{2} \mathrm{~N}$ ) and -acceptors (carbonyl). 10 is shown as a representative. The donors and acceptors were introduced to exert the captodative effect ${ }^{95}$ to stabilize radical resonance structures for the moieties that they sandwich. With a pair of such sandwiched moieties, the diradical character of $\mathbf{1 0}$ is enhanced. The authors calculated low-lying excitation energies for the 5 designed structures using the second order Complete Active Space Perturbation Theory (CASPT2) method with active spaces that span all $\pi$ 
and lone pair orbitals. Such large active spaces can only be used for small molecules. Only 10 was found to satisfy Eqs. 6 and almost satisfy Eq. 5 (close to isoergic SF), as well as $E\left(S_{1}\right)<E\left(T_{2}\right)$. Its small size shall be emphasized, especially when compared with tetracene (2) and pentacene (3).

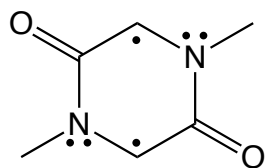

10

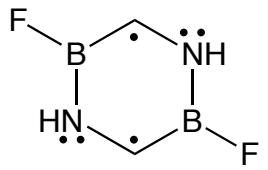

11

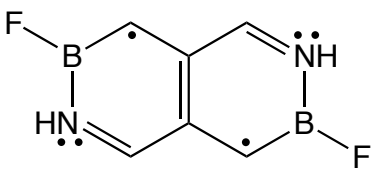

12

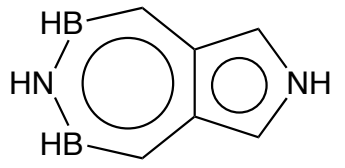

13

The success of the captodative strategy motivated Zeng et al. to follow the same route, but employ a special acceptor, the endocyclic sp ${ }^{2} \mathrm{~B}$ atom. ${ }^{96}$ The authors proposed a series of azaborine(BN)-substituted mono- and bi-cyclic aromatic structures. They calculated excitation energies of the structures using the General Multi-Configurational Quasi-Degenerate Perturbation Theory (GMC-QDPT). Three structures (11 to 13) were found to be promising chromophores, and the smallest 11 is even smaller than 10. Disregarding the methyls on $\mathrm{N}$ in $\mathbf{1 0}$ and recognizing the similarity between $\mathrm{BF}$ and $\mathrm{CO}, \mathbf{1 0}$ and $\mathbf{1 1}$ are isoelectronic and isosteric. Through this work, the authors attempted to crosslink the two vibrant fields of singlet fission and azaborine chemistry. ${ }^{97}$

In Refs. 94 and 96, the two captodatively stabilized radical centers are not in contact. For instance, 11 can be viewed as composed of two BN-substituted methyls, in which the $\mathrm{C}$ sites are not connected. Wen et al. approached the problem in another direction. ${ }^{98}$ They started with two radical centers in contact, i.e., they are covalently coupled, and then reduced the coupling and increased their diradical character by introducing donors and acceptors. The reduction in interaction is illustrated in Figure 6, where we compare the $\pi$-bonding orbitals of the plain (upper) and a captodatively modified (lower) ethylene. The acceptor (donor) delocalizes the $\pi$ singly occupied orbital of methylene through having bonding (antibonding) interaction with the central $\mathrm{C}$. The resultant smaller amplitudes at the central $\mathrm{C}$ atoms reduce $\pi-\pi$ overlap, and hence a smaller HOMO-LUMO gap is obtained. Based on this idea, the authors examined 14 structures that can be viewed as captodatively-modified ethylenes. Their excitation energies were calculated at the CASPT2 level. Three structures (14 to 16) were found to satisfy (or close to satisfy) Eqs. 5 and 6. The authors pointed out the possible complications of $\mathbf{1 5}$ and 16 due to their possible tautomerizations to $\mathbf{1 5}^{\prime}$ and $\mathbf{1 6} \mathbf{6}^{\prime}$. On the other hand, the low $E\left(T_{1}\right) \mathrm{s}(0.63$ and $0.67 \mathrm{eV})$ of $\mathbf{1 4}$ and $\mathbf{1 5}$ may imply instabilities and the 
difficulty to extract electrons from their triplet excitons. With its ideal $E\left(S_{1}\right)=2.23 \mathrm{eV}$ (absorption of solar photons with high irradiance) and $E\left(T_{1}\right)=1.12 \mathrm{eV}$ (close to the optimal $1 \mathrm{eV}$ gap for electron injection), the authors considered $\mathbf{1 6}$ to be the most promising design, if the potential difficulties of tautomerization, formation of hydrogen bonds, and proton transfer can be overcome. The molecules contain CT character in their $S_{0}$-to- $S_{1}$ and $-T_{1}$ excitations. Their $E\left(S_{1}\right) \mathrm{s}$ and $E\left(T_{1}\right) \mathrm{s}$ are hence dependent on solvent polarity. Solvent polarity is hence a handle to tune SF exoergicity of chromophores with CT character in the excitations.
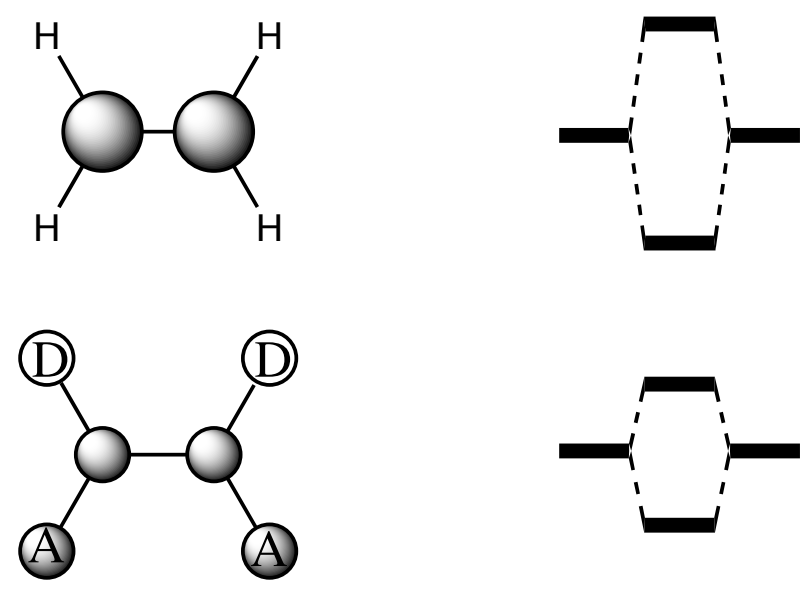

Figure 6. Weakening of covalent interaction between two methylene radicals in forming the $\pi$ bond of ethylene when they contain acceptor (A) and donor (D) substituents.

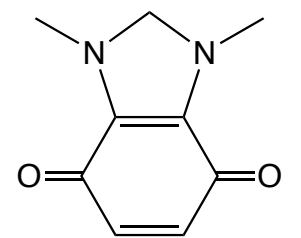

14

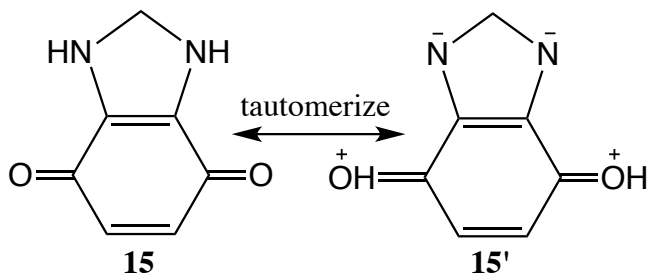

15

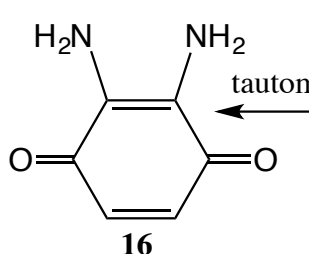

16

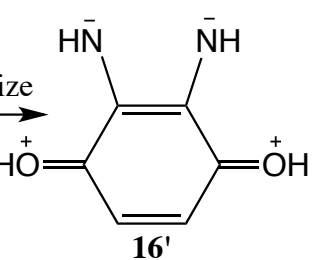

Through density functional theory (DFT) and time-dependent density functional theory (TDDFT) calculations, Bhattacharyya et al. designed a small SF chromophore based on mono-Si-substitution on anthracene $(\mathbf{1 7}) .{ }^{99} \mathrm{Si}$ is an electron-donor and its endocyclic substitution raises HOMO energy more than LUMO energy. The reduced HOMO-LUMO gap increases the diradical character of $\mathbf{1 7}$ and makes it satisfy Eq. 5, with $E\left(S_{1}\right)=2.65$ and $E\left(T_{1}\right)=0.97 \mathrm{eV}$. The authors further introduced a CN group to 17 and the resultant structure (18) was shown to have almost isoergic SF, with $E\left(S_{1}\right)-2 E\left(T_{1}\right)=0.05 \mathrm{eV}$. 


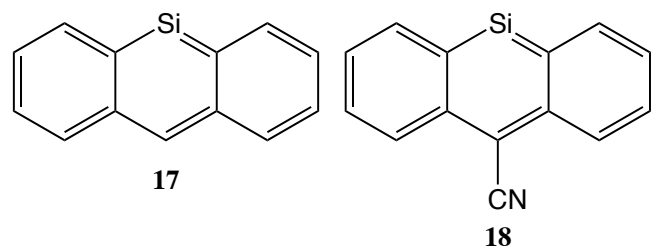

\subsection{Chemically Modified Pentacenes}

In addition to designing new structures, another way to enrich the arsenal of SF chromophores is to chemically modify known chromophores and enhance their stability. Typical SF chromophores like tetracene and pentacene suffer rapid degradation. When they are exposed to air and light, they undergo photooxidation easily. ${ }^{100,101}$ Chen et al. proposed to introduce endocyclic $\mathrm{sp}^{2} \mathrm{~N}$ atoms and thiophene rings to enhance the acenes' stabilities. ${ }^{102}$ The HOMO and LUMO energies may be lowered by the more electronegative heteroatoms. With the lower LUMO energy, the rate of electron transfer from the LUMO to ${ }^{3} \mathrm{O}_{2}$, a mechanism to oxidize the acenes in $S_{1}$ state, is reduced. ${ }^{103}$ The lower HOMO energy can reduce the possibility for singlet oxygen sensitization, another photooxidation mechanism. ${ }^{104}$ However, the SF-exoergicities of the acenes should not be altered by the substitutions. Chen et al. performed (TD)DFT calculations for a series of N-substituted and thiophene-fused tetracenes and pentacenes. They found that the N-substitutions in general lowered both HOMO and LUMO energies, which implies enhanced stability. The orbital energy lowering can be explained by the first order perturbation theory and N's stronger electronegativity than C. On the other hand, the N-substitutions make SF less exoergic for pentacene and even endoergic for tetracene. The less SF exoergicity or even endoergicity arises from the orbital localization (i.e., ionic character) introduced by N. In correspondence, the diradical character is reduced. The thiophene rings induce more complicated modifications of the orbital energies, $E\left(S_{1}\right)$, and $E\left(T_{1}\right)$, depending on their number, position (middle or terminal), and connection patterns $((\alpha, \beta)$ or $(\beta, \beta)$, see 19 and $\mathbf{2 0}$ for example). Considering all factors, the authors concluded that $\mathbf{2 1}$, which has been synthesized, ${ }^{105}$ is the most promising SF chromophore among all studied thiophene-fused structures.

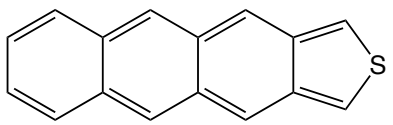

$19(\beta, \beta)$ connection

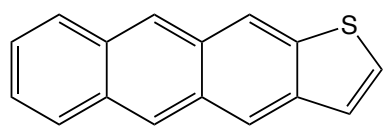

$20(\alpha, \beta)$ connection

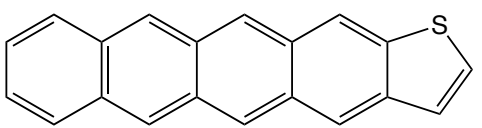

21 
In a follow-up study, Shen et al. systematically investigated the effects of exocyclic substitutions on tuning stability and excitation energies of pentacene. ${ }^{106}$ The authors performed (TD)DFT calculations for a series of mono-exocyclic-substituted pentacenes (see 22 for example), exhausting all four substitution sites ( $\alpha-\delta$ in 22). As expected, electronwithdrawing substituents lowered both HOMO and LUMO energies, stabilizing the substituted pentacenes. Strong $\pi$-acceptors like $\mathrm{CN}$ and $\mathrm{NO}_{2}$ reduced the $\mathrm{SF}$ exoergicity. We believe this is due to the orbital localization and the correspondingly reduction of diradical character. Electron-donating groups raise HOMO and LUMO energies and shall be avoided. The substitution sites play an important role in determining the modification of the orbital energies, because of the site-specific orbital amplitudes and steric hindrances. The authors also looked into the effects of introducing triisotropylsilylethynyl (TIPS, see $\mathbf{2 3}$ for the pentacene with a TIPS at the $\alpha$ position) and a series of S-containing groups to pentacene. The TIPS-substitutions lowered HOMO and LUMO energies in all positions. Only the TIPSsubstitution at the $\alpha$ site increased the SF exoergicity, while the others kept it invariant. The S-containing groups act as $\sigma$-acceptors and $\pi$-donors. The $\sigma$-acceptor character dominated and the S-containing groups stabilized pentacene and reduced SF exoergicity, like the other investigated electron-withdrawing groups. Among all studied S-containing structures, 22 has the lowest LUMO energy and its SF exoergicity is the closest to pentacene. The authors hence considered it to be a very promising SF chromophore for further investigations.
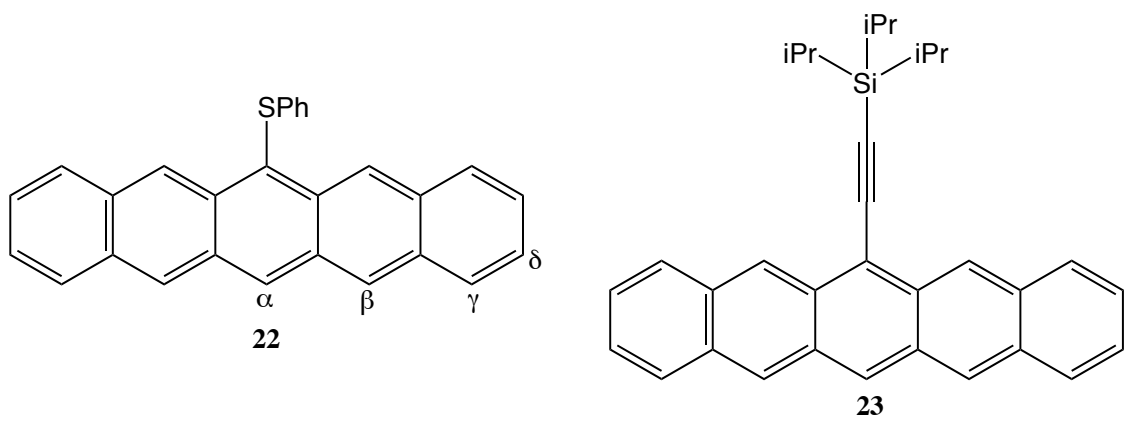

\subsection{Summary of the Promising Theoretical Designs}

The calculated excitation energies of the promising SF chromophores discussed above are summarized in Table 1 for comparison. Among them, $\mathbf{5}(\mathrm{n}=2), \mathbf{1 0}, \mathbf{1 2}, \mathbf{1 6}, \mathbf{1 8}$, and 21 are of special interest. Their $E\left(T_{1}\right) \mathrm{s}$ are close to the optimal $1 \mathrm{eV}$ gap and $E\left(S_{1}\right) \mathrm{s}$ are close to 
twice the $E\left(T_{1}\right) \mathrm{s}$, minimizing energy loss in SF. Also, the close to $2 \mathrm{eV} S_{0}$-to- $S_{1}$ absorption occurs at the frequency range with fairly high spectral irradiance in the solar spectrum. ${ }^{107}$ They are high value targets for future syntheses and experimental investigation. The 19 chromophores in the table were proposed based on different motivations. With hindsight, they can all be viewed as diradicaloids.

Table 1. Excitation energies (in eV) of theoretically designed promising SF chromophores.

\begin{tabular}{ccccccccccc}
\hline Structure & $E\left(S_{1}\right)$ & $E\left(T_{1}\right)$ & $E\left(T_{2}\right)$ & \multicolumn{1}{c}{ Ref. Structure } & $E\left(S_{1}\right)$ & $E\left(T_{1}\right)$ & $E\left(T_{2}\right)$ & Ref. \\
\hline $\mathbf{4}$ & 3.01 & 1.41 & 3.16 & 84 & $\mathbf{1 2}$ & 1.99 & 0.97 & 2.82 & 96 \\
$\mathbf{5}(\mathrm{n}=2)$ & 2.29 & 1.10 & 2.33 & 86 & $\mathbf{1 3}$ & 2.03 & 0.84 & 3.00 & 96 \\
$\mathbf{5}(\mathrm{n}=3)$ & 1.88 & 0.80 & 1.78 & 86 & $\mathbf{1 4}$ & 1.06 & 0.63 & 2.21 & 98 \\
$\mathbf{7}(\mathrm{n}=2)$ & 2.14 & 0.64 & 1.78 & 89 & $\mathbf{1 5}$ & 1.36 & 0.67 & 2.43 & 98 \\
$\mathbf{8}$ N2-4 & 2.60 & 1.08 & 2.75 & 92 & $\mathbf{1 6}$ & 2.23 & 1.12 & 2.74 & 98 \\
$\mathbf{8}$ N0-3 & 2.41 & 1.10 & 2.51 & 92 & $\mathbf{1 7}$ & 2.65 & 0.87 & & 99 \\
$\mathbf{9}-\mathrm{O}$ & 2.80 & 1.36 & 2.92 & 93 & $\mathbf{1 8}$ & 2.57 & 1.26 & & 99 \\
$\mathbf{9}$ '-F & 2.52 & 1.26 & 2.72 & 93 & $\mathbf{2 1}$ & $\sim 2$ & $\sim 1$ & & 102 \\
$\mathbf{1 0}$ & 2.11 & 1.14 & 3.52 & 94 & $\mathbf{2 2}$ & 1.74 & 0.75 & & 106 \\
$\mathbf{1 1}$ & 3.01 & 1.34 & 5.29 & 96 & & & & & \\
\hline
\end{tabular}

\subsection{A DFT-Based Protocol for Efficient Structural Search}

Evidently, the calculation of low-lying excited states takes the most important role in searching for SF chromophores. The post-Hartree-Fock methods that have been used in the aforementioned studies, e.g., CASPT2 and GMC-QDPT, are satisfactorily accurate. However, they are too resource-demanding to be used in extensive screening for chromophores. This limitation motivated Grotjahn et al. to develop a DFT-based protocol to calculate the key excitation energies. ${ }^{108}$ The authors selected the 11 structures studied in Ref. 98 as models. They first employed the CC2 method to calculate their vertical $E\left(S_{1}\right) \mathrm{s}, E\left(S_{2}\right) \mathrm{s}$, $E\left(T_{1}\right) \mathrm{s}$, and $E\left(T_{2}\right) \mathrm{s}$, and extrapolated them to the complete basis set limits. These were the references to evaluate the DFT performances. The authors then used 20 exchange- 
correlation (XC) functionals to calculate these energies, with the TDDFT, TDDFT with the Tamm/Dancoff approximation (TDA), and $\triangle \mathrm{SCF}$ formalisms. Through comparing the DFT energies and the references, the authors found the Lh12ct-SsifPW92 and the M06-2X functionals to give the best agreements with the references, and the former is preferred because of its lower empiricism. The authors concluded that TDDFT shall be used to calculate $E\left(S_{1}\right), E\left(S_{2}\right)$, and $E\left(T_{2}\right)$, while $\triangle \mathrm{SCF}$ for $E\left(T_{1}\right)$. The def2-TZVPD basis set was shown to be adequate. This protocol will enable more extensive searches for SF chromophores in the future.

\section{EXPLORATION FOR SINGLET FISSION MECHANISMS THROUGH ELEC- TRONIC STRUCTURE CALCULATIONS}

The electronic structure studies introduced in the previous section were concentrated on monomers of chromophore candidates. The studies introduced in this section were dedicated to investigate detailed SF processes, which involve at least a dimer of chromophores, unless one chromophore is able to accommodate a pair of triplets. While dimer models are more relevant for intramolecular SF (iSF), they provide fundamental knowledge for the more complicated SF in solids. For instance, the 5 diabats can find their counterparts in solids,

although the number of each type of diabats increases. The couplings between the diabats play similar roles in solids. Also, while state-of-the-art quantum chemistry methods have been applied to investigate SF in dimers, their formidable costs prevent their direct use in solids. Therefore, SF studies in solids very often relied on model Hamiltonians with parameters calculated for dimers. We should be aware of the indispensability and limiations of dimer and small oligomer models in SF research.

\subsection{Studies Using Oligomer Models}

\subsubsection{Transfer integrals and singlet fission, an early study}

Greyson et al. carried out very likely the first electronic structure study of SF in dimers of chromophores. ${ }^{109}$ They investigated the effects of inter-chromophore couplings on SF. They pointed out the three effects of the couplings: (1) large couplings are beneficial for 
fast eg(ge)-to-tt conversion; (2) too large couplings can significantly adjust the eg(ge)-tt energy relations and make SF less thermodynamically favorable; (3) too strong couplings result in undesired binding of the triplet-pair and impede the fission. The first and the other two effects are opposite; one needs to balance them in designing dimer structures that give appropriate transfer integrals. The authors performed (TD)DFT calculations for 47 homodimers of three chromophores: tetracene (2), 1,3-diphenylisobenzofuran (4), and 3-dicyanovinylidene-6-(2'-imidazolidinylene)-1,4-cyclohexadiene (24). They focused on the SF exoergicity and the transfer integrals between HOMOs $\left(t_{H H}\right)$ and LUMOs $\left(t_{L L}\right)$ of the monomers. $t_{H H}$ was approximated as half of the splitting of a dimer's HOMO and HOMO-1, which mainly consist of the symmetric and antisymmetric combinations of the monomers' HOMOs. Similarly, $t_{L L}$ was approximated as half of the splitting of a dimer's LUMO and $\mathrm{LUMO}+1$. The SF exoergicity was approximated as

$$
\Delta E_{S F}=2 E\left(T_{1}\right)-E\left(S_{1}\right)+t_{H H}+t_{L L} .
$$

$t_{H H}$ and $t_{L L}$ were taken to be positive in this work. Their addition to $\Delta E_{S F}$ reflects that the gap reduction, arising from the (de)stabilization of a dimer's HOMO (LUMO) compared to the monomers' counterparts, reduces SF exoergicity. $t_{H L} \mathrm{~S}$ were not calculated and could be reasonably approximated to have similar magnitudes as $t_{H H} \mathrm{~s}$ and $t_{L L} \mathrm{~s}$. The transfer integrals ranged from close to zero to $0.5 \mathrm{eV}$. They may change the SF thermodynamics to less exoergic or even endoergic. Examining the transfer integrals of experimentally known SF dimers, the authors concluded that the integrals need to reach $0.1 \mathrm{eV}$ to give competitive SF. Containing donors and acceptors, 24 was found not to have similar HOMO and LUMO amplitudes at all sites, which is different from the alternant hydrocarbons like $\mathbf{4}$ and the phenyls in 2. Consequently, disparate $t_{H H}$ and $t_{L L}$ were obtained for dimers of $\mathbf{2 4}$. For homodimers, Eq. 2 becomes

$$
V_{e g, t t} \approx \sqrt{\frac{3}{2}} \frac{t_{H L}\left(t_{L L}-t_{H H}\right)}{\Delta E_{C T}}
$$

The chromophore subscripts "A" and "B" are henceforth omitted in the transfer integral labels when no confusion is induced. Disparate $t_{H H}$ and $t_{L L}$ are beneficial for alleviating the destructive interference manifested by the subtraction. 


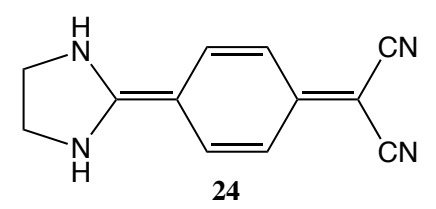

\subsubsection{Quantum chemistry calculations of noncovalent oligomers of acenes and their derivatives}

Kuhlman et al. employed a combined quantum mechanics and molecular mechanics (QM/MM) method to study a pentacene dimer embedded in the pentacene crystal environment (Figure 7). ${ }^{110}$ The $\pi-\pi$ interaction only occurs in the $a-b$ plane of the crystal and hence considering the 2-D structure is enough. The unit cell dimer was treated using (TD)DFT calculation, and it was immersed in a force field of the surrounding molecules. The force field included atom-centered electrostatic potentials and polarization effects. The calculations showed that the lowest singlet excited state of the dimer shared the same structure as the ground state, indicating that no excimer is formed. This finding is against the explanation of the pentacene pump-probe experiments that the photo-induced absorptions (PIAs) arise from an excimer state. ${ }^{5}$ Based on the energy shifts of triplet-to-triplet excitations between pentacene in solutions and in crystals, the authors also did not think that the PIAs arise from independent $T_{1}$ state. Instead, they conjectured that the PIAs arise from a bound triplet-pair state.
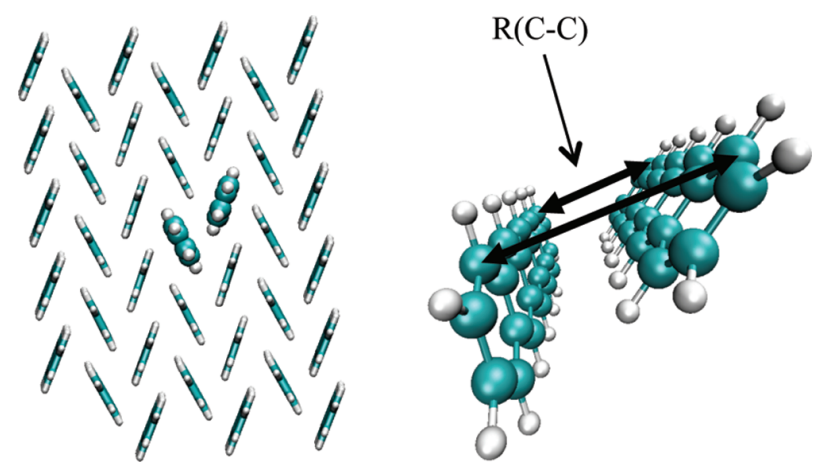

Figure 7. The herringbone crystal structure of pentacene (left) and the inter-molecular distance along which analysis of adiabats was carried out in Ref. 111 (right). The unit cell dimer is highlighted in the left panel. The other acenes have similar herringbone crystal structures. This figure is taken with permission from Ref. 111; Copyright 2011, American Chemical Society.

Zimmerman et al. investigated the low-lying excited states of pentacene molecule using 
the Multi-Reference Møller-Plesset (MRMP) perturbation theory method. ${ }^{112}$ They proposed that there is an optically "dark" ${ }^{1} A_{g}$ state below the lowest optically "bright" ${ }^{1} B_{2 u}$ state, and the dark state possesses triplet-pair character and mediates the SF in pentacene. However, this finding is inconsistent with the fact that the fluorescence of pentacene in the ${ }^{1} B_{2 u}$ state is not quenched by a lower lying dark state. ${ }^{1}$ A follow-up study ${ }^{113}$ showed that this energy ordering stems from an inadequate active space in the calculations and the ensuing intruder state problem. ${ }^{114,115}$

Zimmerman et al. calculated the low-lying excited states for tetracene and pentacene clusters with up to 10 molecules using TDDFT. ${ }^{111}$ They found that the $S_{1}$ states of the clusters were mostly concentrated on four adjacent molecules, and as the inter-molecular distance (Figure $7(\mathrm{~b})$ ) between the two central molecules was reduced, the exciton was further localized onto the two molecules. This suggests a formation of an excimer. The permanent dipole moments of the low-lying singlet states of a reduced cluster, which contains the four molecules on which the $S_{1}$ states are localized, were found to be small. The authors thus concluded that there is little CT component in those states, and therefore the CTmediated SF is not effective in the two acenes. This conclusion is inconsistent with most of the other studies (see below). As a matter of fact, the lack of a large permanent dipole moment does not necessarily indicate the lack of CT character. ${ }^{116}$ A dimer state that contains similar contributions from CT states of opposite polarities, e.g., ca and $a c$, also exhibits a small dipole. The authors investigated the adiabatic state mainly of $t t$ character (the $D$ state in their notation) for the central dimer of each of the acenes using the efficient Restricted Active Space Double Spin-Flip (RAS-2SF) method with the minimum 4o4e active space. They compared the $D$ state potential energy curve with those of the other low-lying singlet states along the inter-molecular distance. For the pentacene dimer, they found the crossing of the $D$ and $S_{1}$ states at a shorter distance. Along the distance shortening, the energy of the bright $S_{1}$ state decreases, a result of forming the excimer, while the $D$ energy increases. The photo-excited dimer can hence readily reach the crossing region. The authors employed the Landau-Zener-Stueckelberg theory ${ }^{117,118}$ to calculate the $S_{1}$-to- $D$ transfer probability to be $20 \%$ at the crossing region. The easiness of reaching the crossing region and the fairly high transfer probability were combined to explain the efficient SF in pentacene. More advantages of using the RAS-2SF method in studying SF were elaborated in Ref. 119.

Beljonne et al. used the INDO/SCI and INDO/CCSD methods to calculate the excited 
states of the crystal unit cell pentacene dimer model. ${ }^{120}$ They tuned the CT energies and consequently the CT percentages in the lowest bright adiabats through using a screening factor for the electron-electron repulsion. They found that only when the CT percentage in the lowest adiabat reaches $50 \%$ the calculated Davydov splitting agrees with the experimental value. This is consistent with what they found earlier. ${ }^{121}$ Such a substantial mixing between $\mathrm{CT}$ and $e g(g e)$ diabats facilitates the superexchange eg(ge)-tt coupling. The authors also found that the herringbone packing geometry of the pentacenes in crystal induces large transfer integrals in Eqs. 1 and 2, and hence strengthens the eg(ge)-tt coupling.

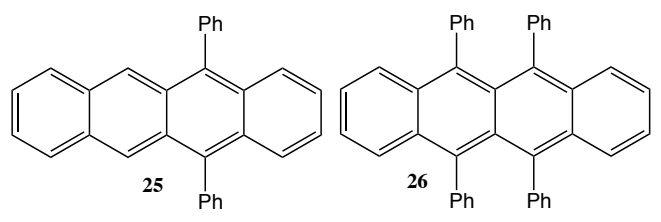

Casanova carried out an electronic structure study of SF in tetracene and its two derivatives, 5,12-diphenyltetracene (DPT, (25)) and rubrene (26). ${ }^{122}$ He first compared his TDDFT excitation energies and oscillator strengths of the three species with experimental results and validated the methodology. The substitutions did not modify the frontier orbitals and the characters of the low-lying excited states significantly. The bulky substituents, however, do change the packing morphology. He then calculated excitation energies of clusters of the three species and confirmed the exciton delocalization to more than 7 monomers, as observed in experiment. The author investigated the low-lying excited states of dimer models and found high-lying CT states in all three cases; the CT states could only participate in SF through the superexchange mechanism, and they were found to play a more significant role in tetracene than in the two derivatives. The author also found that along the distortion towards the SE-optimized structure, SE and ME states approach in energy. The distortion is hence a plausible catalyst for SF.

In a subsequent paper, Casanova investigated a "forgotten" phenomenon, bright fission $(\mathrm{BF})$, the fission of a high-lying singlet excited state to two lowest-lying singlet excited states: ${ }^{123}$

$$
S_{0} S_{n} \rightarrow S_{1} S_{1}
$$

The pair of symbols on each side denote the composite state of two interacting chromophores. $S_{n}$ stands for a high-lying singlet state generated by photoexcitation. This generation of two 
emitting states from absorbing one photon is very likely to find its use in lighting applications. Although this is not a SF phenomenon, the two share many aspects in common. The author gave a comprehensive overview on BF, including possible states that are involved, possible competing deactivation channels, and overall requirements on having efficient BF. For instance, the fast internal conversion from $S_{n}$ to $S_{1}$ in one chromophore, which is predicted by Kasha's rule, is detrimental for BF; CT states are likely to be close in energy to $S_{0} S_{n}$ and hence play a more significant role in BF than in SF. The author used anthracene as a model to elaborate some aspects about BF, e.g., energy matching between single-excitonic and multi-singlet-excitonic states, CT contributions to multi-singlet-excitonic states, qualitative estimates of nonadiabatic transition rates from single- to multi-singlet-excitonic states, the effects of delocalization of the single-excitonic state, and the effects of inter-chromophore conformations. Possible high-lying single-excitonic states for efficient BF in anthracene were identified. This work is a good starting point for the renaissance of research in BF.

Zeng et al. used the Multi-Configurational Quasi-Degenerate Perturbation Theory (MCQDPT) method to systematically investigate the low-lying excited states of pentacene and their roles in SF. ${ }^{113}$ They first studied the monomer. Their calculated vertical $E\left(S_{1}\right)$ and minimum-to-minimum $E\left(T_{1}\right)$ were 2.31 and $0.86 \mathrm{eV}$, in very good agreement with experimental values. They found the $S_{1}$ state to be the bright ${ }^{1} B_{2 u}$ state corresponding to one-electron HOMO-to-LUMO excitation. This contradicts the finding in Ref. 112, in which the dark ${ }^{1} A_{g}$ state with multi-excitonic character was identified as the $S_{1}$ state using the same method. Zeng et al. attributed this difference to the intruder states problem ${ }^{114,115}$ in the calculation in Ref. 112. The authors also studied the unit cell pentacene dimer. The negligible ( 1 meV) eg(ge)-tt couplings and the more significant (> $64 \mathrm{meV}$ ) eg(ge)-ca(ac) and $t t-c a(a c)$ couplings indicate the CT-mediated mechanism for SF in pentacene. Due to the asymmetry of the two monomers, one CT state was found to lie $0.8 \mathrm{eV}$ lower than the other and it was mainly this state that mediates the efficient SF. The authors also found the CT state to be more active in mediating the SF when the inter-monomer distance was shortened. They hence proposed to compress pentacene crystal along a certain direction to further improve the SF efficiency.

Coto et al. employed the CASPT2 method and the many-body Green's Function approach (GW/BSE) to study the low-lying excited states of pentacene oligomers. ${ }^{116}$ They also found the monomeric $S_{1}$ state to be the bright ${ }^{1} B_{2 u}$ state. They pointed out that $4 \mathrm{o} 4 \mathrm{e}$ 
is the minimum active space to give accurate CASPT2 energies for the monomer. The authors then proportionally enlarged the active space to study the low-lying states of pentacene dimers and trimers with crystal packing conformations. Trimer conformations allow further delocalization of SE states. For all the dimer and trimer structures, the ME-dominated adiabats were all found to lie higher than those with SE and CT characters. The authors hence concluded that the efficient SF in pentacene is driven by significant geometry reorganization that lowers the ME energy. The authors also showed that GW/BSE gave similar results as CASPT2 in describing SE and CT states. Once it is extended to describe ME states, GW/BSE will be a very useful method in studying SF. Especially, it can describe excitons in real solids. ${ }^{124}$

Bhattacharyya and Datta investigated the role of packing geometry on SF in TIPSanthracene (similar to the monomer in $\mathbf{3 1}$ discussed below, but with an anthracene core). ${ }^{19}$ Through (TD)DFT calculations, the authors found $E\left(S_{1}\right)=2 E\left(T_{1}\right)=2.72 \mathrm{eV}$ for TIPSanthracene, i.e., an isoergic SF. The authors investigated dimers with 6 packing geometries (PI-A to -D, PII-E, and PII-F) in the two phases of TIPS-anthracene polymorphs, PI and PII. The direct eg(ge)-tt couplings were found to be surprisingly large, e.g., $130 \mathrm{meV}$ for the PII-F dimer. This is larger than all direct couplings ever reported, and larger than all one-electron transfer integrals between the frontier orbitals of the two monomers in PII-F. On the other hand, the CT states lied about $1.2 \mathrm{eV}$ higher in energy than eg and ge, a result of the far inter-chromophore distances ranging from 8 to $10 \AA$. Given such far distances, the large direct eg(ge)-tt couplings are even more impressive. With all these results, the authors concluded that the SF in TIPS-anthracene occurs through a direct mechanism. The authors employed the Marcus theory formula to calculate the SF time scales to be 3.56 and $1.3 \mathrm{ps}$ in PI and PII. These are comparable with the experimentally reported ps SF time scale in TIPS-pentacene. ${ }^{125}$ The large direct $e g(g e)-t t$ couplings are worth further investigation. They only consist of two-electron integral contributions (The first row in Eq 1). There may be some unknown reason for those two-electron integrals being so large.

\subsubsection{A transferable model}

Yost et al. employed the Constrained DFT with Configuration Interaction (CDFT-CI) method to prepare the five diabats for dimers of 10 materials: tetracene, pentacene, hex- 
acene, and some of their substituted species. ${ }^{126}$ The authors diagonalized the four diabats excluding $t t$ and calculated the coupling between the so-obtained lowest bright state and $t t$ :

$$
\bar{V}=\langle\text { Bright }|\hat{H}| t t\rangle .
$$

$\bar{V}$ contains both the direct and the CT-mediated eg(ge)-tt couplings. Adapting the Bixon and Jortner model ${ }^{127}$ for electron transfer to $\mathrm{SF}$, the authors proposed a unified kinetic model to estimate the $\mathrm{SF}$ rate:

$$
\begin{aligned}
k_{S F} & =\sum_{n} \frac{\bar{V}^{2} k_{n}}{1+\tau_{n}^{a d} \bar{V}^{2}} ; \\
k_{n} & =\left(\frac{\pi}{\hbar^{2} \lambda k T}\right)^{1 / 2}|\langle 0 \mid n\rangle|^{2} e^{-\frac{(\Delta G+n \hbar \omega+\lambda)^{2}}{4 \lambda k T}} \tau_{n}^{a d}=\frac{4 \pi}{\hbar \lambda} \tau_{a d}|\langle 0 \mid n\rangle|^{2} .
\end{aligned}
$$

$\Delta G$ stands for the energy driving force for SF, $\lambda$ the reorganization energy, the unsubscripted $k$ the Boltzmann constant, $n$ the quantum number of the characteristic vibrational mode that connects the bright state and $t t, k_{n}$ the nonadiabatic rate for transition to $t t$ with $n$ vibrons, and $\tau_{a d}$ the adiabatic SF time scale when $\bar{V}$ is large and the adiabatic transition limit is reached. This rate expression becomes the Marcus theory nonadiabatic expression at the small $\bar{V}$ limit:

$$
k_{S F} \approx \frac{2 \pi}{\hbar} \bar{V}^{2} \frac{1}{\sqrt{4 \pi \lambda k T}} e^{-\frac{(\Delta G+\lambda)^{2}}{4 \lambda k T}},
$$

and reaches the adiabatic limit at the large $\bar{V}$ end. Substituting the calculated $\bar{V}$ s and the other parameters for the 10 materials in the expression, the authors calculated their $k_{S F} \mathrm{~S}$ and the results were in line with the experimentally measured values. The 10 materials span over a large range from small to large $\bar{V}$, and from negative to positive $\Delta G$. The robustness of this model is evident. Through analyzing the dependence of the calculated $k_{S F} \mathrm{~s}$ on $\bar{V}$ and $\Delta G$, the authors concluded that $E\left(S_{1}\right)-2 E\left(T_{1}\right)$ being slightly negative $(\Delta G \lesssim 0)$ is the most desirable for efficient SF chromophores. Too much exoergicity brings the transition to the inverted region of the Marcus theory. This was confirmed by the slower SF in hexacene than in pentacene (530 vs. 100 fs). ${ }^{128}$ In Ref. 128, Busby et al. developed a microscopic rate expression and interpreted this slower SF as a multi-phonon relaxation effect. Yost et al. also pointed out that increasing the coupling will reach an adiabatic limit, where the rate is no longer dependent on $\bar{V}$. The work significantly deepened our knowledge of SF by clarifying the different roles of the energy gap and the coupling between SE and ME states. 
The 10 materials test set were used by Yang and Hsu to examine their Fragment Spin Difference (FSD) scheme in calculating the effective eg(ge)-tt couplings. ${ }^{129}$ In the FSD scheme, the diabats are generated by localizing the electronic spins to the corresponding fragments. The authors employed this diabatization scheme onto the adiabats obtained using the SpinFlip Configuration Interaction Singles (SF-CIS) method to construct SE and ME diabats. CT components were incorporated in the diabats. The calculated couplings were in qualitative agreement with those obtained by Yost et al, ${ }^{126}$ manifesting the usefulness of this convenient first principle diabatization scheme. The CT percentage in SE state is correlated with the coupling strength, reflecting the dominance of the CT-mediated coupling.

\subsubsection{SF in covalent dimers of tetracene and pentacene}

Damrauer and coworkers carried out a series of studies on intramolecular SF of homodimers of tetracene and its derivatives. The tetracene-based chromophores were connected by norbornyl, a bridge commonly used in studies of electron transfer. In the first paper of this series, Vallett et al. used (TD)DFT methods to calculate low-lying states relevant to the 5 diabats for norbornyl-connected tetracene dimers. ${ }^{21}$ The five dimers that they investigated are three $C_{2 v}$ structures that connect the tetracenes at their short edges and with different bridge lengths (27, called BT1 to BT3), and the two that have a cis and a trans arrangement of the tetracenes and the shortest norbornyl bridge (28, called BT1-cis and BT1-trans). A natural finding was that the electronic coupling between the two tetracenes is reduced as the norbornyl length is increased. The SF thermodynamic driving force was found to be largely invariant with respect to the length, as the two tetracenes are relatively isolated. In BT1-cis and -trans, the two monomers are either closer to each other or have their frontier orbitals delocalized to the norbornyl bridge. Consequently, both the driving force and the couplings can be modified. Specifically, the $C_{2 v}$ symmetry of BT1-3 nullifies $t_{H L}$. The $e g(g e)-t t$ couplings can only be induced by symmetry-breaking vibrations. This constraint does not apply to the $C_{2}$ BT1-trans and $C_{s}$ BT1-cis. Also with their lower CT energies, the authors considered BT1-trans and -cis as promising SF chromophores.

To further investigate the feasibility of vibronic-induced SF in BT1, Alguire et al. cal-

culated $t_{H L}$ as a function of the molecule's vibrational coordinates. ${ }^{42}$ The authors first developed a method to obtain monomer-centered frontier orbitals. The authors first localized 


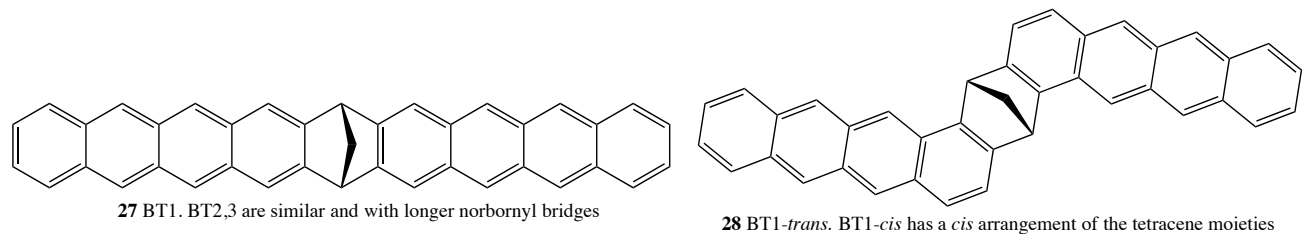

the canonical orbitals on the monomers using the Boys scheme. ${ }^{130,131}$ The total Fock matrix was projected onto the localized orbital space and then diagonalized to give the monomeric HOMO and LUMO, which give the transfer integrals. The authors used this method to calculate $t_{H L}$ of BT-1 along its vibrational modes of $A_{2}$ and $B_{2}$ irreducible representations (irreps), which lower the symmetry to $C_{2}$ and $C_{s}$, respectively. The lower symmetries make $t_{H L}$ nonzero. The gradients of the two elements are of the same sign along the $b_{2}$ modes and opposite signs along the $a_{2}$ modes. Considering the signs of the other static transfer integrals, the $b_{2^{-}}$induced $\left(a_{2^{-}}\right) \mathrm{SF}$ features a destructive (constructive) interference between the two CT-mediated branches. The $a_{2}$-induced vibronic-SF is hence more pronounced. The authors also pointed out the importance of the low frequency modes in vibronic-SF because of their larger fluctuations at room temperature. In the end, the authors incorporated their calculated coupling gradients into the Stuchebrukhov model ${ }^{132}$ and examined the relation between the thermally averaged effective $e g(g e)$-tt couplings and the reorganization energy. The thermally averaged effective couplings vary from 5.4 to $5.8 \mathrm{meV}$ for BT1 as the reorganization energy changes from 100 and $1000 \mathrm{meV}$. This magnitude of effective coupling is comparable to that of tetracene. Therefore, BT1 is likely to undergo SF like tetracene. Overall, this study revealed the importance of the Peierls coupling in SF: the effective $e g(g e)-t t$ couplings can solely arise from vibronic interaction. This argument was confirmed by the coherent SF observed in a transient absorption study on rubrene crystal. ${ }^{133}$

Instead of relying on the thermal fluctuations of the $a_{2}$ and $b_{2}$ vibrations, one may permanently lower the symmetry by modifying the connection between the tetracene and the norbornyl. BT1-trans and BT1-cis are two such structures. In the third paper of this series, ${ }^{134}$ Damrauer and Snyder evaluated the effective $e g(g e)-t t$ couplings to be 1.1 and $2.0 \mathrm{meV}$ for BT1-trans and -cis, respectively. Despite the small magnitudes of all transfer integrals in BT-1-trans, the constructive interference between the two CT branches gives an effective coupling comparable to that of BT1-cis. Using the Marcus theory and the typical $130 \mathrm{meV}$ reorganization energy of tetracene, the authors estimated the SF time scale to be 19 and 5.2 ps for BT1-trans and -cis, respectively, or 80.9 and 23.2 ps when a 
$300 \mathrm{meV}$ reorganization was used to include the contribution from solvent. The symmetrylowering can also be realized by heteroatom substitution. The authors investigated two sets of aza-substituted BT1, aza-BT1-2 and aza-BT1-3; within each set there are a trans and a cis conformation, with the trans being shown in $\mathbf{2 9}$ and $\mathbf{3 0}$. The two trans conformations had both larger magnitudes of the transfer integrals and the constructive interference. Consequently, the eg-tt effective couplings were calculated to be 1.5 and $9.9 \mathrm{meV}$ for azaBT1-2-trans and aza-BT1-3-trans, an order of magnitude larger than the 0.12 and $0.59 \mathrm{meV}$ cis counterparts. Putting these couplings and the $130 \mathrm{meV}$ reorganization energy into the Marcus theory rate formula, the authors evaluated the SF time scales to be 84 and 1.5 ps for aza-BT1-2-trans and aza-BT1-3-trans, and $12 \mathrm{~ns}$ and $430 \mathrm{ps}$ for the corresponding cis structures. This study clearly demonstrated the feasibility of controlling SF efficiency in covalent chromophore dimers by modifying symmetry.
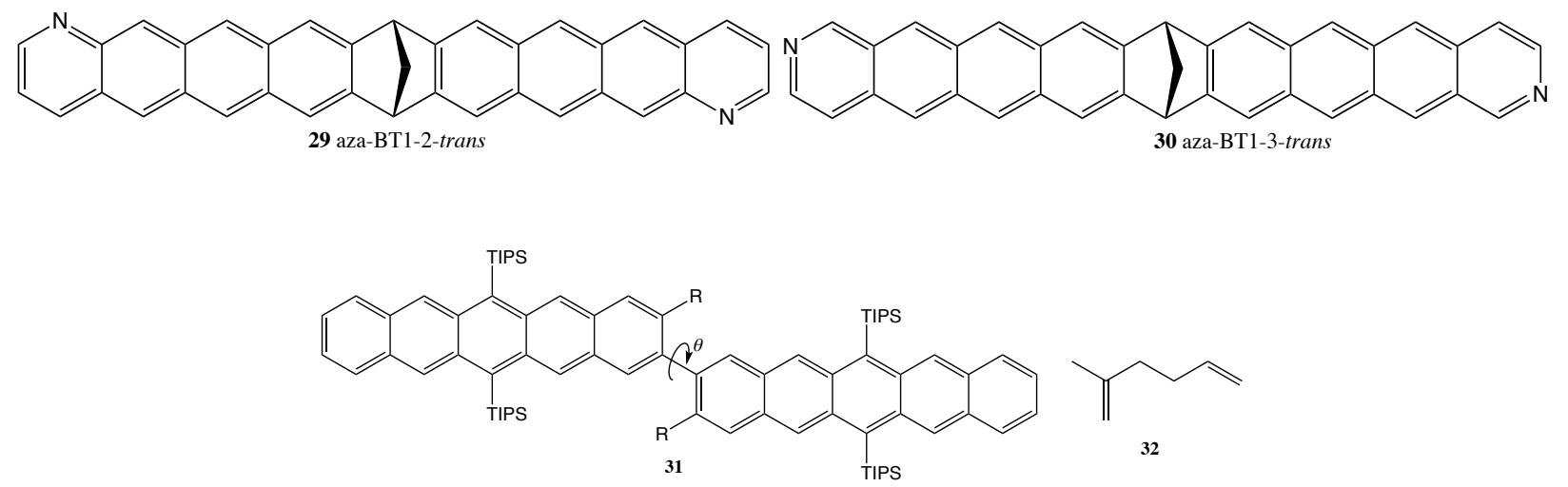

Covalently connected pentacene dimers exhibit similarly fast SF dynamics as in pentacene crystals. ${ }^{29-31}$ However, a different SF mechanism may apply. The short-edge-connected bipentacenes studied by Sanders et al. did not display solvent polarity dependence in their SF, ${ }^{29,30}$ implying negligible roles of any CT states. To unravel the mechanism, Fuemmeler et al. did a quantum chemistry study on the bi-TIPS-pentacenes shown in $31 .{ }^{135}$ Their MCQDPT calculation for 31 with $\mathrm{R}=\mathrm{H}$, called BP-37 due to its $37^{\circ}$ dihedral angle $\theta$, showed that the CT states are too high lying to play any role in its intramolecular SF. This is reasonable due to the long distance $(14 \AA)$ between the geometric centers of the separated charges in the CT states, compared to the $4 \sim 5 \AA$ counterpart in crystalline pentacene. However, they found a crossing between the eg(ge) and $t$ diabats in the forward direction of a $1435 \mathrm{~cm}^{-1}$ vibrational mode, which is a symmetric combination of the two monomers' motions shown in Figure 8(A). There is a corresponding exchange of diabatic 
characters between the lowest three adiabats, which is shown in Figure 8(B). This indicates that despite the weak direct $e g(g e)$-tt couplings, the acute avoided crossings of the adiabats dominated by the different excitonic states lead to an efficient nonadiabatic SF. The fact that the crossings occur at the forward region is of paramount importance. After the photoexcitation to the pseudo-degenerate $S_{1}$ or $S_{2}$ (only one is bright), BP-37 lowers its energy along the vibrational mode and reaches the crossing point, and hence transit to the $t t$-dominated adiabat. The more substantial decrease of the $t$ energy along the vibrational mode is explained by Figure $8(\mathrm{C})$. The bond shortenings denoted by the red arrows result in two Clar's sextets in the second and the fourth rings, leaving a diradical resonance structure at the middle ring, which favors low triplet energy. And this vibronic energy lowering is doubled for $t t$ because it occurs in both monomers. Increasing the dihedral angle $\theta$ to $57^{\circ}$, the direct $e g(g e)-t t$ couplings are reduced, and based on the Fermi's golden rule, the $\mathrm{SF}$ rate shall be slower by 4 times. This prediction was consistent with the experimentally determined 4.5 times slower SF of BP-2Ph (with R being a phenyl group in 31 and having $\left.\theta=57^{\circ}\right)$.

\subsubsection{Using nonorthogonal configuration interaction to study SF}

Havenith et al. employed the Complete Active Space Self-Consistent Field (CASSCF), Multi-Reference Configuration Interaction (MRCI), CASPT2, and Non-Orthogonal Configuration Interaction (NOCI) methods to study SF using two models, a 2-methyl-1,5-hexadiene molecule (32) and a tetracene trimer. ${ }^{136}$ The two vinyls in $\mathbf{3 2}$ were viewed as chromophores and the authors selected a $4 \mathrm{o} 4 \mathrm{e}$ active space comprising the $\pi$ orbitals and electrons. They calculated low-lying singlet excited states that enclose $t t, e g, g e$, and one CT state. The energy order is sensitive to the treatment of dynamical correlation. At the CASSCF level, the $t t$-dominated adiabat is lower than the eg/ge-dominated ones; SF is favoured. However, once the dynamical correlation is included at the MRCI and CASPT2 levels, the $t t$-dominated state lies higher. The $t t$-dominated adiabat and the quintet state of pure triplet-pair character shared similar structures and vibrational frequencies. One can then approximate the former by the latter in geometry optimization and hessian calculations at inexpensive open-shell DFT level. The NOCI calculation was carried out for the tetracene trimer. Each monomer was described using a 4o4e active space. The authors showed that 

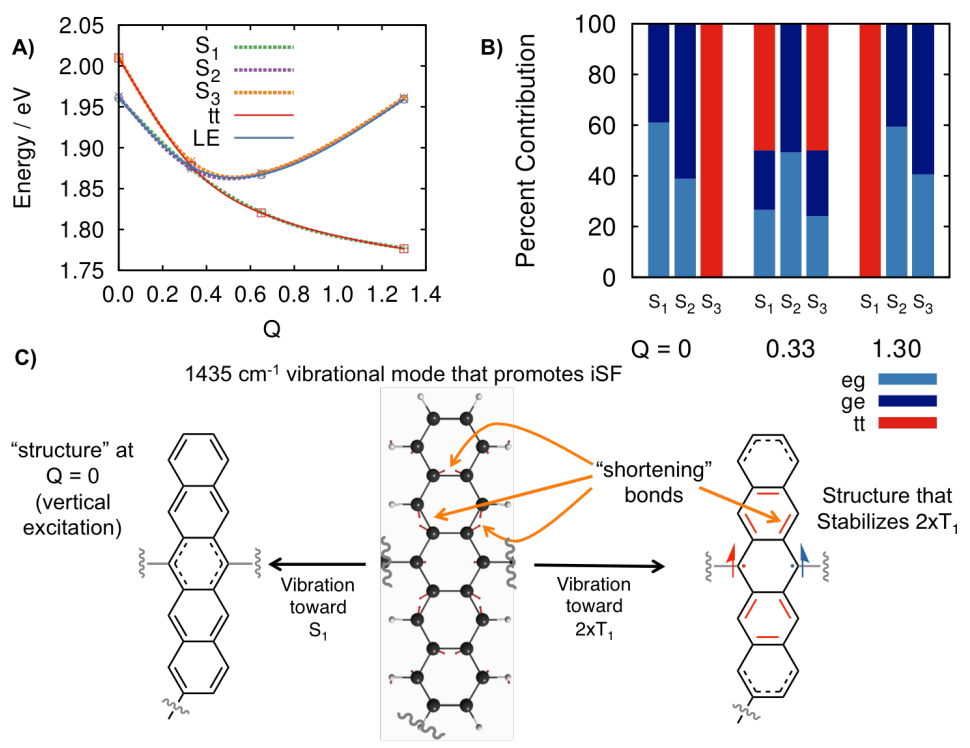

Figure 8. (A) Adiabatic $\left(S_{n}\right)$ and diabatic $(L E$ and $t t)$ potential energy curves of BP-37 along the $1435 \mathrm{~cm}^{-1}$ symmetric mode's coordinate $Q$. eg and ge are grouped together and labelled by $L E$ (local excitons). (B) Percent diabatic contributions to the lowest three singlet excited states at three representative $Q$ coordinates. (C) Illustration of the $1435 \mathrm{~cm}^{-1}$ mode's motion on one TIPS-pentacene monomer. This figure is taken with permission from Ref. 135; Copyright 2016, American Chemical Society.

the CT states play an important role in delocalizing the single-excitonic states. The trimer model allows for delocalization of the three triplet-pair states. However, they were found to be dimer-localized. As expected, the CT states was found to facilitate SE-ME coupling.

More recently, Wibowo et al. improved the NOCI method by allowing orbital relaxation for each state. ${ }^{137}$ They employed this method to evaluate the effective eg(ge)-tt couplings for the model chromophore designed by Akdag et al. (10). ${ }^{94}$ They first used conventional DFT method and periodic boundary conditions to optimize the crystal structure for the chromophores, which has two types of $\pi$ stacking, called Stacks A and B. The authors then constructed three dimer models, two for the two stackings and one for the inter-stack conformation, and calculated the inter-state couplings for the 5 diabats of each dimer. As in Ref. 136, the CT states enhanced the SE-ME couplings from $<5 \mathrm{meV}$ to $\sim 17 \mathrm{meV}$ for the two intra-stack dimers. For the inter-stack dimer, the coupling was weak because of the insignificant $\pi-\pi$ overlaps of the two inter-stack monomers. 


\subsubsection{Using the norm of transition density matrix to study SF}

Feng et al. studied SF in tetracene and pentacene dimer models. ${ }^{138}$ They employed the RAS-2SF method in their calculations and analyzed the wave function compositions of the 5 lowest-lying excited states. A central conclusion is that diabatic representation is not capable of describing SF with quantitative accuracy because the contributions of each of the 5 prototype diabats do not add up to $100 \%$ in the 5 excited states. This conclusion was disputed by Berkelbach et al, ${ }^{139}$ who attributed the $<100 \%$ summation to the incompleteness of the multielectronic basis set. We agree with Feng et al. that the 5 diabats model is a qualitative and pedagogical description of SF. However, we do not think that this imperfection is equivalent to the inaptness of the diabatic representation in SF studies. Diabats are defined to be slowly varying states with respect to molecular structures and are related to adiabats by a unitary transformation. ${ }^{18}$ As long as a group of adiabats mainly exchange characters within themselves, they can be transformed to a set of diabats. This is the essence of the Group (or Generalized) Born-Oppenheimer Approximation (GBOA). ${ }^{140,141}$ The diabats are not necessarily of pure characters of the five prototypes. As long as they can still be characterized as, e.g., eg-dominated and $t$-dominated diabats, the 5 diabats model applies. To invalidate the model, one needs to demonstrate a significant portion of diabats other than the 5 enter the low-lying adiabats, and they open new pathways to mediate the $e g(g e)-t t$ couplings. Even if so, we can enlarge the diabatic space to include those states.

Feng et al. also proposed to use the norm of the one-electron transition density matrix between the adiabats that consist of SE and ME characters as a qualitative estimation of the nonadiabatic SF rates. ${ }^{138}$ The same group of authors used an ethylene dimer model to demonstrate that while intermolecular nonadiabatic couplings follow the trend of the norm divided by the energy gap between the relevant states, intramolecular counterparts display more complicated behaviours. ${ }^{142}$ In the end, a large norm is only a necessary but not a sufficient condition for a large nonadiabatic coupling.

Chan et al. observed SF in crystalline tetracene although this is an endoergic process. ${ }^{7}$ They attributed the SF to the entropic gain in the process. Realizing the importance of entropy, Kolomeisky et al. proposed a minimalist SF kinetic model that consists of three states: delocalized SE state (state 0), ME state with the triplet-pair residing on adjacent (state 1) and separated (state 2 ) molecules. ${ }^{39} \mathrm{SF}$ was approximated as the process converting 
from state 0 to state 1 (step 1 ) and then to state 2 (step 2). For state 0 , there are a number of ways to find adjacent molecules that accommodate the triplet-pair and therefore, entropy increases in step 1. In step 2, there are a number of ways for two adjacent triplet excitons to separate. Therefore, step 2 also features an entropy increase. Considering the structure of acene crystals, the authors calculated the entropies of the two steps. They both increase as state 0 becomes more delocalized, and the increase of entropy in step 2 is greater than in step 1. These entropy gains drive endoergic SF to occur. The authors defined the tripletpair binding energy as the energy difference between states 1 and 2 . This is another handle to tune SF efficiency. When it is too large, step 1 is efficient while step 2 is not; when it is too small, the opposite situation occurs. There is hence a material-specific optimal binding energy for SF. Using this model, Kolomeisky et al. estimated the SF time scales for tetracene and pentacene. They explained the 3 orders of magnitude slower SF in tetracene.

Feng et al. adapted this model and the norm of transition density matrix to calculate relative SF rates for a series of dimers of 1,3-diphenylisobenzofuran (4), 1,6-diphenyl-1,3,5hexatriene (33), and 5,12-diphenyltetracene (25). ${ }^{143}$ The dimer structures reflect different morphologies. The relative rates of $\mathbf{4}$ and $\mathbf{3 3}$ agree well with experiments and therefore, the relative rates of $\mathbf{2 5}$ give confident predictions of the SF in its two crystals. The morphology effects on SF were all related to the inter-chromophore interactions that modify the CT percentages in SE and ME states, and the Davydov splitting of the two SE states. More recently, Feng and Krylov used the same model to calculate relative SF rates for a series of covalently linked tetracene dimers, ${ }^{144}$ with respect to neat tetracene. The dimers include three phenylene-connected structures $(\mathbf{3 4 - 3 6})^{145}$ and two cofacial alkynyltetracene dimers (BET-B 37 and BET-X 38). ${ }^{32}$ The rates of the phenylene-connected dimers are much slower than those of BET-B and BET-X. These calculated results agree well with experimental findings. The authors analyzed the similarities and differences between SFs of BET-B and -X, as well as the effects of the linkers. In the last paper of this series, Feng et al. used this model to investigate $\mathrm{SF}$ in $(\mathrm{BET}-\mathrm{B})_{2}$ dimer. ${ }^{146}$ The relative $\mathrm{SF}$ rates in $(\mathrm{BET}-\mathrm{B})_{2}$ and BET-B are similar. Through analyzing low-lying states of (BET-B $)_{2}$, the authors found that the intramolecular ME states were lower in energy and contained more CT character. These factors speak for the dominant intramolecular nature of the SF in (BET-B) 2 , and in BET-B crystal as well. 


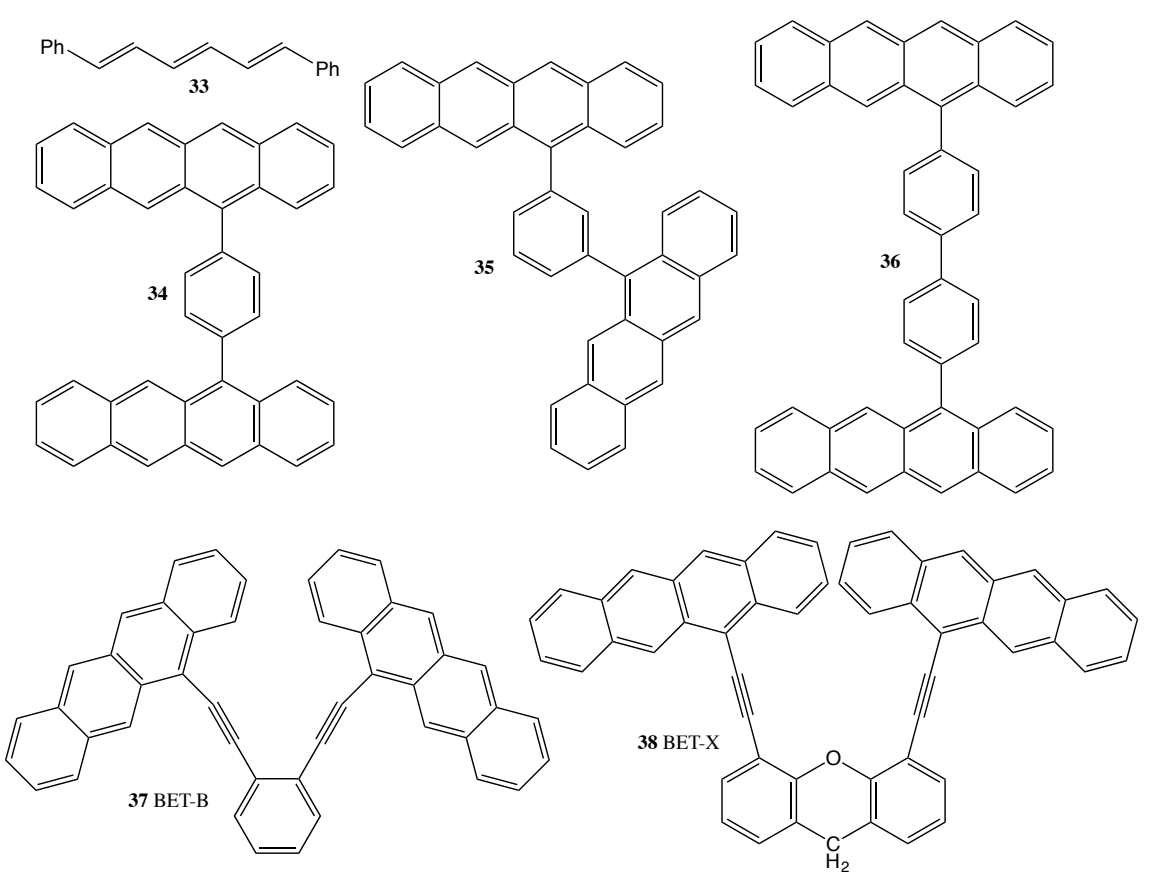

\subsubsection{Using the inexpensive PPP Hamiltonians to study SF}

Aryanpour et al. employed the inexpensive semiclassical PPP Hamiltonian and Configuration Interaction with up to quadruple excitations (QCI) to calculate low-lying states of oligomers of polyenes, acenes, and covalently connected acene dimers. ${ }^{147}$ For the octatetraene oligomers, the authors investigated the hypothetical eclipsed, the slip-stacked, and the carotenoid monoclinic geometries. The lowest-lying ME states were all found to have the triplet-pair residing on different molecules. The packing geometry plays a decisive role in determining whether such an intermolecular SF occurs in octatetraene. While the lowest singlet excited state mainly contains ME character in the eclipsed dimer and trimer, it is composed of SE and CT characters in the eclipsed tetramer. Therefore, the eclipsed geometry is SF-unfavorable, and the importance of the size of oligomer is demonstrated. For the slip-stacked and the monoclinic geometries, the lowest singlet excited states are all MEdominated; SF is exoergic. These findings are beneficial for understanding SF in polyenes. The authors also analyzed the low-lying singlet states of tetracene dimers and trimers and pentacene dimers with the crystal packings. The sharing of CT character in SE and ME facilitates SF. For the tetracene and pentacene dimers connected by a para-phenylene (34 and the pentacene analogue), the CT states were far away in energy from SE and ME and they were not active in mediating SF. The authors hence gave an explanation for the low 
SF yield ${ }^{148,149}$ in the tetracene dimer.

Aryanpour et al. employed the same method to investigate iSF in donor-acceptor copolymers. ${ }^{150}$ The authors first simplified the donor-acceptor copolymer, poly[2,7-(5,5-bis-(3,7dimethyl octyl)-5H-dithieno[3,2-b:2',3'-d]pyran)-alt-4,7-(5,6-difluoro-2,1,3-benzothiadiazole)] (PDTP-DFBT), into a cis-polyene model (Figure 9). The strengths of donor, acceptor, and electron correlation were represented by the PPP site energies $\epsilon_{A, A^{\prime}, B, C}$. The authors fixed the $\epsilon_{A, A^{\prime}}$ values and the $\epsilon_{B} / \epsilon_{C}$ ratio; $\epsilon_{B}$ was the only parameter to tune the donor-acceptor strength and the CT character in HOMO-to-LUMO excitation. When all the $\epsilon_{A, A^{\prime}, B, C}$ were set to zero, the model became a trans-dodecahexaene of $C_{2 v}$ symmetry. While the ME state was lower in energy than the SE state, they were of different irreps and did not mix. When the acceptor-donor strength was turned on, the symmetry was broken, the electron correlation was increased, the two states mixed, and the ME-dominated adiabat acquired oscillator strength; SF was enabled. As the acceptor-donor strength increased, the SE state decreased in energy as it gained more CT character. There is hence an optimal donoracceptor strength: it is large enough to give oscillator strength to the ME-dominated state so that it is directly populated by photoexcitation; it is not too large to make the SE-CT state lower in energy than the ME-dominated state. The fact that the ME-dominated state became a bright state explained the two close-lying ground state absorptions and the two distinct transient photo-induced absorptions observed in different donor-acceptor copolymers. $^{26,151,152}$

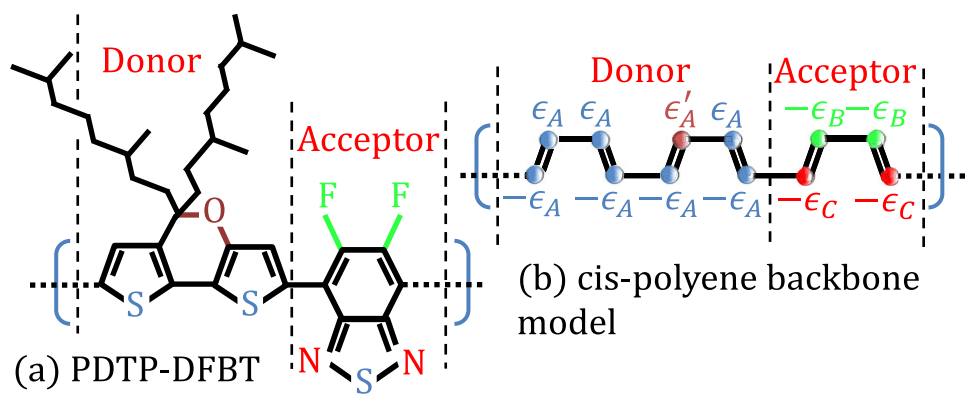

Figure 9. The donor-acceptor copolymer (a) and its simplified polyene model (b) studied in Ref. 150. This figure is taken with permission from Ref. 150; Copyright 2015, American Physics Society.

Ren et al. studied the low-lying excited states of the donor-acceptor-type conjugated copolymer of benzodithiophene-thiophene-1,1-dioxide (PBTDO, 39). ${ }^{153}$ They described the 
polymer using a PPP model and calculated the excited states using the Density Matrix Renormalization Group (DMRG) method. They found that the lowest singlet excited state is a dark ${ }^{1} A_{g}$ state, which is close in energy to the bright ${ }^{1} B_{u}$ state and contains a strong triplet-pair character. This character and the easiness of decoupling the triplets are enhanced by the donor-acceptor push-pull strength. The ${ }^{1} A_{g}$ state was hence concluded to be an important intermediate for iSF in the polymer. The pseudo-degeneracy between the ${ }^{1} B_{u}$ and the ${ }^{1} A_{g}$ states facilitates SF. The authors also estimated and compared the rates of the ${ }^{1} A_{g}$ state decaying to the ground state in polymer and monomers. The slower rate of the polymer explains that iSF is observed in the polymer but not in the monomers.

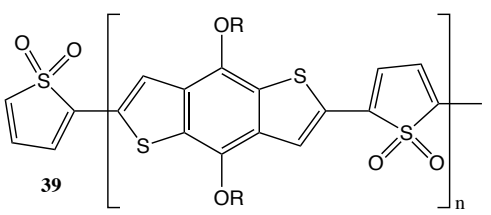

\subsubsection{Solid state effects on charge-transfer energies}

Petelenz et al. questioned the large $0.8 \mathrm{eV}$ difference between $E(c a)$ and $E(a c)$ in Ref. 113: it may have been an artifact of treating the dimer without considering its solid environment. They developed a Self-Consistent Charge Field (SCCF) method to embed the dimer in a cluster of 10 pentacenes and calculated the $E(c a)$ and $E(a c)$ of the dimer under the electrostatic potential of the surrounding molecules. ${ }^{154}$ The resultant energies were extrapolated to the infinite crystal limit. ${ }^{155} E(c a)$ was found to be substantially stabilized by the environment and $E(c a)-E(a c)$ was reduced to $0.01 \sim 0.2 \mathrm{eV}$ (depending on computational details). Using Eq. 2, we calculate the effective eg-tt coupling to be -48 and $-26 \mathrm{meV}$, with the $E(C T) \mathrm{s}$ in Ref. 113 and the most different ones $(E(c a)=2.45$ and $E(a c)=2.28 \mathrm{eV})$ in Ref. 154, respectively. The destructive interference is enhanced by the solid surrounding. This study highlighted the limitation of using an isolated dimer to simulate SF in solid. Using dimer models is a common approximation. The authors suggested to use effective CT energies with implicit solid effects to construct the 5 diabats model.

With the parametrized CT energies, Petelenz and Snamina calculated and analyzed the electronic states of pentacene clusters with $2,10,24$, and 44 molecules on the $a-b$ plane of the pentacene crystal (Figure 10). ${ }^{156}$ The electronic diabats include SE states of all molecules, 
one ME state at the center, CT states at adjacent molecules, and CT states with the electron and hole separated by one unit cell-length along the $a$ or $b$ direction. Once the cluster's size reaches 10 molecules, the lowest adiabat is not dominated by $t t$, despite $t t$ 's lowest energy among the 5 diabats of the central dimer. $t t$ is dispersed in the semi-continuum of adiabats that are mixtures of SE and CT states. This result seems inconsistent with the finding in Ref. 38, where the lowest adiabat was found to be dominated by ME. This is due to the different diabatic energies used in the two works. In Ref. 38, the ME energy is for a pair of pentacenes with their $T_{1}$-optimized structure. Such a low ME energy $(1.75 \mathrm{eV}$, compared to $1.9 \mathrm{eV}$ used in Ref. 156) makes ME dominate the lowest adiabat. Combining the results in Refs. 38 and 156, we see that upon the vertical excitation, the pentacene solid is in an interaction region, where SE, ME, and CT states fully interact and mix; the vibronic coupling then relaxes the pentacene structures, destroys the coherence between the diabats, and eventually concentrates the triplet-pair character on the two molecules distorting towards the $T_{1}$-structure.

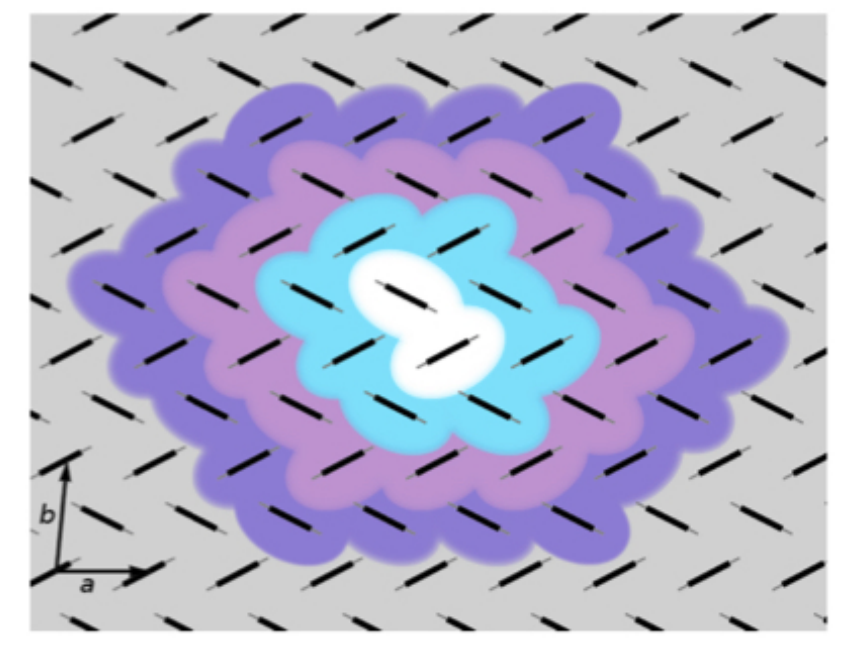

Figure 10. Pentacene clusters that were calculated in Ref. 156. Extended from the white zone to the violet enclosure, the clusters include 2, 10, 24, and 44 molecules. This figure is taken with permission from Ref. 156; Copyright 2015, American Chemical Society.

One way to have asymmetry in the CT energies is to place the dimer at surface or at interface. Petelenz and Snamina constructed a model cluster with 32 pentacenes and used the SCCF method to parametrized CT energies. ${ }^{157}$ Similar diabats as in Ref. 156 were used, with the ME state being localized on a dimer at the boundary of the cluster that mimics the 
$(1,1,0)$ surface of the pentacene crystal. SF efficiency was enhanced compared to placing the ME state in the bulk. Further increase was obtained when the cluster was interfaced to other clusters simulating differently oriented crystallites. Based on these findings, the authors predicted a more efficient SF in polycrystalline than in single crystal pentacene.

Another way to tune the asymmetry in the CT energies is to introduce vacancies or impurities beside the SF dimer. Snamina and Petelenz used the same SCCF method to investigate a 10 pentacenes cluster. ${ }^{158}$ The energies were extrapolated to the infinite crystal limit. ${ }^{155}$ One pentacene was removed from the cluster to simulate a vacancy. This perturbation modified CT energies of all nearby dimers, due to the change of the polarizability in their environments. For some of the dimers, the $E(c a)-E(a c)$ gap is enlarged to $0.40 \mathrm{eV}$. The destructive interference is thus alleviated and SF is more efficient. The authors moved one step further to replace the vacancy by a hypothetical dopant, which may have a strong quadrupole (40) or dipole moment (41, and two more similar $C_{2 v}$ structures with two fewer $\mathrm{N}$ atoms). The dopants lead to more CT asymmetry, especially the dipoles. The SF efficiency can hence be enhanced more. Vacancies and dopants can hence catalyze SF by alleviating the destructive interference.

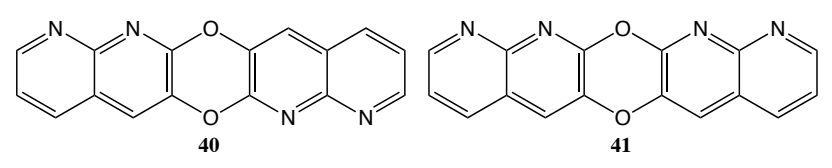

\subsubsection{Construction of the five diabats with more extensive active space}

To reduce the cost of advanced quantum chemistry calculations in SF studies, Parker et al. developed an active space decomposition strategy to construct model Hamiltonians for dimers. ${ }^{159}$ This innovative method contains the following steps: (1) calculation of adiabats for each monomer that are relevant for SF, i.e., singlet and triplet excited states, cationic and anionic states; (2) the adiabats of the two monomers were multiplied to form a dimeric multielectronic basis set. The product states were spin-adapted and grouped according to the 5 types of diabats. Each group contains more than one product state; (3) the dimeric Hamiltonian was expanded within each group of the product states and diagonalized. The eigenstate with the lowest energy was the model state, which was dominated by the corresponding member of the 5 diabats; (4) the dimeric Hamiltonian was expanded in the 5 
model states. The central idea of this method is to minimize the multielectronic basis space for time-consuming dimeric calculations. The authors applied the method to calculate the 5 diabats Hamiltonian matrices for tetracene and pentacene dimers. The matrix elements converged quickly with respect to active space and the number of monomeric adiabats obtained in Step (1). The 5 model states constituted more than $99 \%$ of the dimeric adiabats that were expanded in the full dimeric product states space. The key contribution of this work is a novel scheme to construct the 5 diabats model using a large active space. It can be generalized to handle larger oligomers and include dynamic correlation.

\subsubsection{Using vibronic coupling density to study SF}

Ito et al. calculated the vibronic couplings for the 5 diabats of a tetracene dimer model with respect to all in-phase and out-of-phase combinations of the two monomers' vibrational modes. ${ }^{160}$ Both Holstein and Peierls couplings were included. The vibrational modes that give the most pronounced couplings were identified and analyzed using the concept of vi-

bronic coupling density. ${ }^{161}$ Such an analysis decomposes a vibronic coupling constant into the contributions from the electronic (differential or transition density) and the vibrational part (potential derivative density). Through this analysis, the authors found the importance of the relative phase of the two monomers' vibrations in CT and tt states, which involve electronic structural changes in both molecules. They reconfirmed that the high-frequency CC stretching gives large Holstein coupling for $e g, g e$, and $t t$. Low-frequency acoustic modes were speculated to give large Holstein couplings for the CT states. They were also speculated to give large Peierls couplings as they change intermolecular orbital overlaps.

\subsubsection{Using the Green's function approach to study SF}

Inspired by the recent breakthroughs in intramolecular SF (iSF), ${ }^{28,30-32}$ Ito et al. employed the Green's function approach ${ }^{162}$ and the second-order quasi-degenerate perturbation theory to study the effective transfer integrals between frontier orbitals of chromophore units that are connected by a covalent linker. ${ }^{163}$ Their objective was to propose guidelines to select appropriate linkers and connect them wisely to chromophore units to enhance SF efficiency. 
According to the perturbation theory, the effective eg-tt coupling has the formula

$$
V_{S F}=\frac{1}{2} \sqrt{\frac{3}{2}}\left(\frac{t_{H_{A} H_{B}} t_{H_{A} L_{B}}-t_{L_{A} L_{B}} t_{L_{A} H_{B}}}{E(e g)-E(c a)}+\frac{t_{H_{A} H_{B}} t_{H_{A} L_{B}}-t_{L_{A} L_{B}} t_{L_{A} H_{B}}}{E(t t)-E(c a)}\right) .
$$

The symmetries of $E(e g)=E(g e)$ and $E(c a)=E(a c)$ have been assumed. According to the Green's function method, each of the effective transfer integrals in Eq. 15 contains the direct interaction between the chromophores' frontier orbitals and their mediated interaction through the linker. Considering only the HOMO and LUMO of the linker that are most relevant and at the level of Hückel theory, the mediated transfer integrals can be approximated by

$$
t_{i j}^{\text {mediated }} \approx c_{\mu^{\prime} i} c_{\nu^{\prime} j} \beta^{2}\left(\frac{c_{\mu H_{L}} c_{\nu H_{L}}}{E-E_{H_{L}}}-\frac{c_{\mu L_{L}} c_{\nu L_{L}}}{E_{L_{L}}-E}\right) .
$$

The chromophore units are connected at their $\mu^{\prime}$ and $\nu^{\prime}$ sites to the $\mu$ and $\nu$ sites of the linker, respectively. $c_{\mu i}$ stands for the coefficient of orbital $i$ at site $\mu, H_{L}$ and $L_{L}$ stand for HOMO and LUMO of the linker, $\beta$ is the Hückel's resonance integral between adjacent $\mathrm{C}$ atoms, and $E$ is the Fermi energy that lies between the orbital energies $E_{H_{L}}$ and $E_{L_{L}}$.
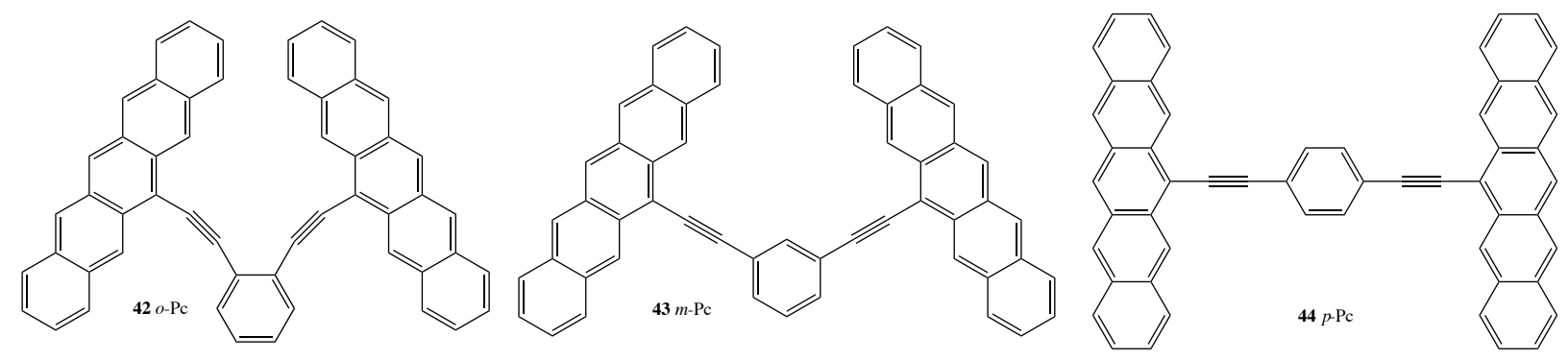

Large $t_{i j}$ s are necessary for large $V_{S F}$. Ito et al. calculated $t_{i j}^{\text {mediated }}$ S for the three diethynylphenyl-connected pentacene dimers $(\mathbf{4 2 - 4 4 )}$ and found that the integrals of $o$ and $p$ - $\mathrm{Pc}$ were significantly larger than those of $m-\mathrm{Pc}$, in consistence with the different $\mathrm{SF}$ rates of the three isomers measured in Ref. 28. Ito et al. explained the difference in $t_{i j}^{\text {mediated }}$ S based on the Coulson-Rushbrooke pairing theorem. ${ }^{164}$ Given an alternant hydrocarbon linker, when $\mu$ and $\nu$ are both unstarred sites, e.g., $43, c_{\mu H_{L}}$ and $c_{\mu L_{L}}$ are of the same sign, and so are $c_{\nu H_{L}}$ and $c_{\nu L_{L}}$. The two numerators $c_{\mu H_{L}} c_{\nu H_{L}}$ and $c_{\mu L_{L}} c_{\nu L_{L}}$ in Eq. 16 are hence of the same sign. The two denominators are always positive. Consequently, the two terms in the parentheses are of the same sign and cancel each other. When $\mu$ is a starred and $\nu$ an unstarred site, e.g., 42 and $44, c_{\mu H_{L}}$ and $c_{\mu L_{L}}$ are of the same sign, while $c_{\nu H_{L}}$ and $c_{\nu L_{L}}$ of opposite signs. Therefore, $c_{\mu H_{L}} c_{\nu H_{L}}$ and $c_{\mu L_{L}} c_{\nu L_{L}}$ are of opposite signs. The subtraction 
in the parentheses in Eq. 16 leads to an accumulation. The authors proposed three ways to tune the interference within the parentheses: using a non-alternant hydrocarbon linker, heterosubstitution on the linker, and using a radical linker.

(a) Head-to-head and head-to-head linked

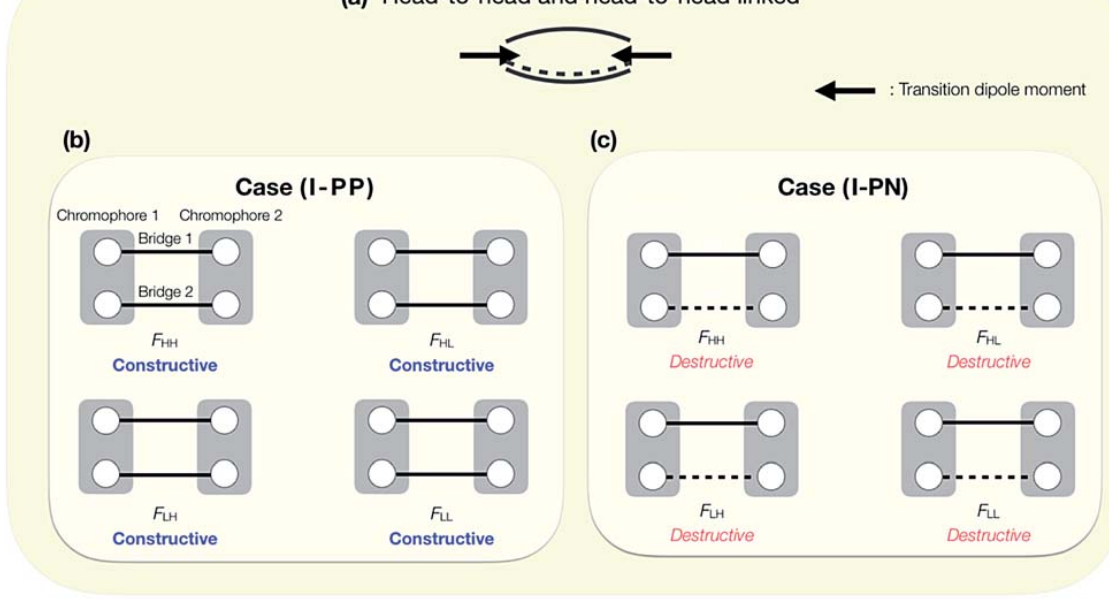

(d) Head-to-head and tail-to-tail linked

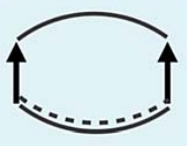

(e)

(f)
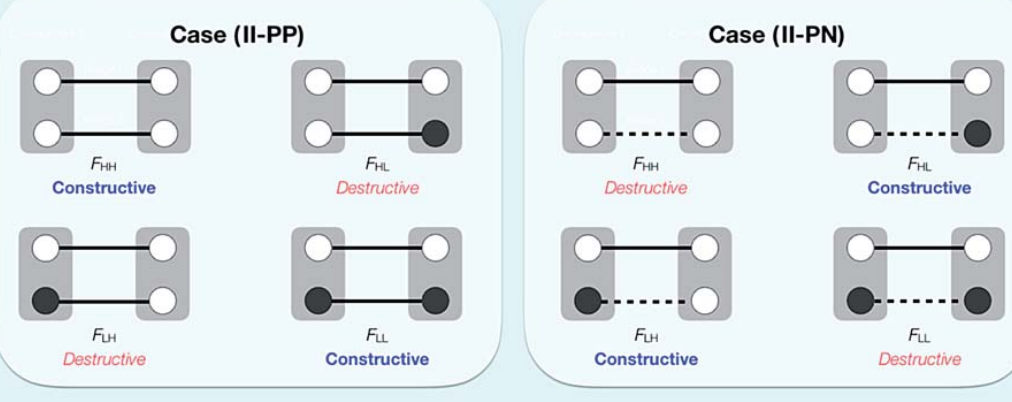

Constructive

Destructive

Figure 11. Schematic representations of the four cases of inter-linker interference discussed in Ref. 165. The phases of the chromophore units' HOMO and LUMO at the connecting sites are indicated by white (positive) and black (negative) circles. All intra-linker interferences are constructive, and their signs are indicated by the solid (positive) and dash (negative) horizontal lines. The phase relations between the HOMO and LUMO can be represented by the transition dipoles (the arrows) in panels (a) and (b). This figure is taken with permission from Ref. 165; Copyright 2017, Royal Society of Chemistry (United Kingdom).

Ito et al. continued applying the same method to investigate quantum interference in 
structures with two chromophore units being connected by two linkers. ${ }^{165}$ The two linkers analogue of Eq. 16 reads

$$
\begin{aligned}
t_{i j}^{\text {mediated }} \approx & c_{\mu^{\prime}(1) i} c_{\nu^{\prime}(1) j} \beta^{2}\left(\frac{c_{\mu(1) H_{L}} c_{\nu(1) H_{L}}}{E-E_{H_{L}(1)}}-\frac{c_{\mu L_{L}(1)} c_{\nu L_{L}(1)}}{E_{L_{L}(1)}-E}\right) \\
& +c_{\mu^{\prime}(2) i} c_{\nu^{\prime}(2) j} \beta^{2}\left(\frac{c_{\mu(2) H_{L}} c_{\nu(2) H_{L}}}{E-E_{H_{L}(2)}}-\frac{c_{\mu L_{L}(2)} c_{\nu L_{L}(2)}}{E_{L_{L}(2)}-E}\right) .
\end{aligned}
$$

Subscripts (1) and (2) denote the linkers. In addition to the intra-linker interference within each pair of the parentheses that is discussed in the previous paragraph, there is also an interlinker interference between the two terms that are summed on the right hand side of Eq. 17. Given constructive intra-linker interferences, the inter-linker interference is determined by the signs of the two terms in Eq. 17, which are determined by the phases of the chromophore units' frontier orbitals at the connecting sites. Ito et al. summarized four cases of the interlinker interference: I-PP, I-PN, II-PP, and II-PN. P and $\mathrm{N}$ denote the positiveness and negativeness of the two parenthesized terms, respectively; I and II denote the two phasecombinations of the chromophore units' HOMO and LUMO at the connecting sites. These four cases are schematically shown in Figure 11. Model systems of tetracene dimer connected by polyynes with various lengths were used to calculate the double-linker-mediated transfer integrals (Fock matrix elements), which were strengthened or weakened by the inter-linker interferences as shown in the figure, in consistence with the analysis based on Eq. 17. How the four patterns affect SF efficiency and yield was discussed.

\subsubsection{Including the charge-transfer states implicitly}

Nagami et al. studied packing effects on SF in oligorylene (5). ${ }^{166}$ They focused on dimer models of terrylene and quaterrylene (5 with $n=2,3)$. A 3 diabats model with $e g$, ge, and $t t$ was employed. The effects of CT states were incorporated by perturbing the three diabats. The authors examined the variations of the transfer integrals, the effective eg(ge)-tt couplings, and the perturbed energies of eg (ge) and $t$, along the change of packing geometry. Two terrylene monomers were placed cofacially as the initial dimer structure, with the inter monomer distance $z=3.41 \AA$, and one monomer on the $x$ - $y$ plane. The in-plane

monomer was then displaced within the plane to give lateral and longitudinal changes of packing geometry. The perturbations of the CT states to the eg $(g e)$ and $t t$ energies are 
dependent on the transfer integrals, and hence dependent on the packing geometry. Balancing the couplings and the energies, the authors proposed several packing geometries that are candidates for efficient SF. The results also shed light on the different SF performances of two real crystal packings of terrylene. A similar study was carried out by Ito et al. for pancake-bonded systems, taking the tetramer of phenalenyl radical (45) as the model. ${ }^{167}$

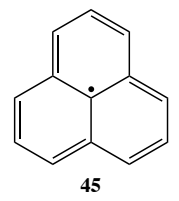

\subsection{Mapping SF Problems to Spin-Interaction Problems}

ME states are of multi-reference nature. It is a formidable task to calculate their energies in a large cluster of chromophores using ab initio methods. The diabatic energies are usually approximated by twice the chromophore $E\left(T_{1}\right) \cdot{ }^{139,168}$ Recently, Mayhall developed a new approach to calculate the ME energies. ${ }^{169}$ The only ab initio calculations needed are for the single reference high spin state with spin multiplicity $2 n+1$ and the 1-electron SpinFlipping Configuration Interaction (1SF-CI) taking the high spin state as the reference. $n$ is the number of chromophores. These calculations are economic enough to be applied to large clusters. The $2 n$ 1SF-CI eigenstates with the lowest energies are projected onto the neutral determinant basis, so that the CT components in those states are treated implicitly. The projected states and the $2 n$ eigenenergies are then used to construct a Bloch effective Hamiltonian, which has the form of a spin-Heisenberg-Dirac-Van Vleck Hamiltonian for $2 n$ spin-1/2 lattice sites. Diagonalizing this Hamiltonian gives the eigenstates dominated by ME character. The central idea of this method is to map each chromophore to two spin1/2 lattice sites, and to select their appropriate couplings that correspond to ME states. The author compared the triplet-pair binding energies, and the energy difference between the triplet-pair states with the total quintet and singlet spins, calculated using the new approach and the ab initio Two-electron Spin-Flipping Complete Active Space (2SF-CAS) method. The comparison was carried out for 11 representative chromophore dimers. The agreement is convincing. When the 1SF-CI step is replaced by a spin-flipping TDDFT calculation, the results were found to be dependent on the percentage of exact exchange in 
the functional. Functionals with more exact exchange or range-separate functionals were shown to be more reliable. The author also pointed out that this approach is inapplicable when there are strong couplings between ME and CT states. This new method is very efficient in describing ME states in large systems, if it is used properly.

In their most recent study, Abraham and Mayhall extended the Ovchinnikov's rule to predict boundedness of the ME state in covalently-linked iSF chromophores. ${ }^{170}$ Their method is illustrated in Figure 12. Like in Ref. 169, the triplet residing on one chromophore (square box in the figure) is represented by two spin- $1 / 2$ lattice sites, which are spin-parallel (of the same color, see the rightmost panel of Figure 12(b)). All the other adjacent sites are of opposite colors to indicate antiferromagnetic interactions of those spins. Whether the sites in the square boxes are of the same (quintet, unbound, ferromagnetic triplet-pair) or opposite (singlet, bound, antiferromagnetic triplet-pair) colors is solely dependent on whether the two connecting sites of the linker are of the same (meta-) or opposite (paraand ortho-phenylenes) colors. The meta- and para-connecting schemes were shown in Figure 12(b). The connection of this simple scheme to Ovchinnikov's rule in judging ferromagnetic/antiferromagnetic coupling between two radical centers is clearly seen by comparing Panels (a) and (b) in the figure. The authors examined this rule by performing ab initio calculations for a series of covalently linked dimers. Good agreements between predictions and calculated results were obtained. The authors pointed out the limitations of this rule: (a) it is only applicable to through-bond coupling between the two chromophores. Through-space coupling in general favors antiferromagnetic triplet-triplet binding; (b) it is only applicable to alternant hydrocarbon linkers. Only those have the appropriate color pattern; (c) the triplet excitons must be localized in the chromophore units.

\subsection{Model Developments for SF in Extended Systems}

Ambrosio and Troisi developed a model that describes SF in linear chains of chromophores. ${ }^{171}$ The electronic Hamiltonian was based on Configuration Interaction with Single and Double excitations (CISD); only HOMO and LUMO of each monomer were considered. This model has encompassed all of the 5 diabats for any adjacent dimers. The authors first employed this model to investigate the dimers and linear trimers of tetracene and petancene. The overall good agreement between their dimer results and those in the other dimer studies 


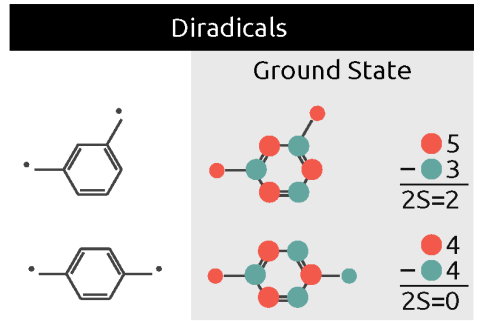

(a)

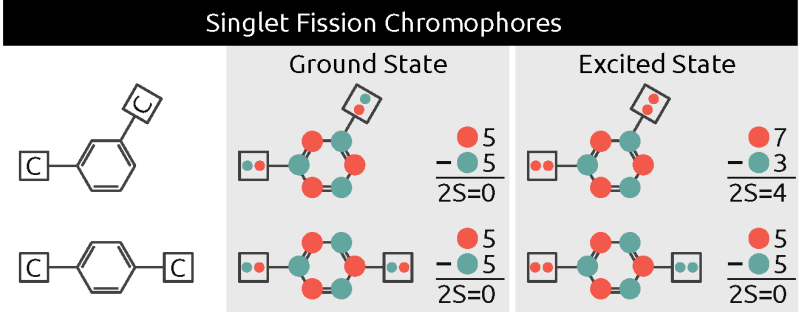

(b)

Figure 12. (a) Ovchinnikov's rule in judging the ground state spin of diradicals; (b) the extension of Ovchinnikov's rule to judge the triplet-pair's overall spin. The square boxes with "C" indicate SF chromophores. This figure is taken with permission from Ref. 170; Copyright 2017, American Chemical Society.

validates the parameters in their model. The trimer calculations resulted in a new ME state with two non-adjacent triplets. Lacking the triplet-triplet binding, this state is higher in energy than adjacent triplets. The former was called MEu (unbound) and the latter MEb (bound). In extending from the trimer to the linear chain model, the authors employed a perturbation theory treatment to incorporate all CT-type states in an effective Hamiltonian. Only SE, MEb, and MEu states were explicitly considered, and their couplings contained CT-mediated contributions. The authors then used the model to study decamer chains of tetracene and pentacene. The large singlet-to-singlet and small triplet-to-triplet transfer integrals resulted in a wide SE and two narrow ME bands, one for MEb and the other for MEu. The narrow ME bandwidths suggest localization of the triplet-pairs by vibronic coupling, while the large SE bandwidth suggests delocalization of the singlet exciton. The latter is consistent with the finding in Ref. 122. The SE states had stronger couplings with MEb states because the couplings were mediated by the lower-lying CT states. As the chain length increased, the couplings decreased (Effect 1), however, each SE state was coupled to more ME states (Effect 2). The authors approximated the vibronic interactions by linearly coupling bath harmonic oscillators to the diabats. They estimated the SF rate based on the Fermi's golden rule. The rate increased with respect to the chain length. Therefore, the aforementioned Effect 2 dominates over Effect 1; exciton delocalization promotes SF. The authors also found that the fission rate to $\mathrm{MEb}$ is faster than to $\mathrm{MEu}$ states, as the former is lower in energy and more strongly coupled to the SE states. This work demonstrates the limitation of small oligomer models in studying SF in solids. 
Shortly after, Teichen and Eaves developed another one-dimensional model to study the collective aspects of SF in molecular crystals. ${ }^{172}$ In this model, the periodic boundary conditions are enforced. The Hamiltonian contains singlet and triplet local excitations. Excitonic couplings between adjacent molecules, $J_{S}$ and $J_{T}$, govern the bandwidths of the excitonic states of the two spin manifolds. The Hamiltonian couples (with the strength $\gamma / 4$ ) each singlet excitation to a pair of adjacent triplet excitations. Adjacent triplet excitations are bound by the strength $\chi$. The authors employed the Jordan-Wigner transformation to convert the Hamiltonian into a fermion representation. The model can then be handled using many-body theory and Green's functions for fermionic systems. The authors then analyzed the coupling between the SE state with momentum $k=0$ (the only bright SE state in this periodic model) to the ME states whose center-of-mass momentum is 0 . These triplet-pair states form a band based on the relative momentum between the triplets. Mapping their model to the Fano-Anderson model, the authors obtained a Fermi's golden rule type formula for the SF rate constant:

$$
\begin{array}{r}
W_{0}=2 \pi|V|^{2}\left(E_{S}\right) \rho\left(E_{S}\right) ; \rho(E)=\frac{1}{8 \pi J_{T}} \frac{1}{\sqrt{1-\delta^{2}}} ; \\
|V|^{2}(E)=\gamma^{2}\left[1-\delta^{2}\right] ; \delta=\left(E-2 \epsilon_{T}\right) / 4 J_{T}
\end{array}
$$

$\epsilon_{T}$ is the triplet energy, and $E_{S}$ the bright SE energy. While the density of states $\rho(E)$ peaks at the triplet-pair band edges with the van Hove singularities $(\delta=1)$, the coupling $V=0$ there. The balance of $\rho$ and $|V|^{2}$ determines that the most significant SF coupling occurs between the bright SE state and the middle of the triplet-pair band. Therefore, a small $J_{T}$, i.e., highly localized triplet excitons, facilitate SF. Comparing the rate $W_{0}$ with that obtained using a SE state delocalized to a less extent, the authors found that the rate decreases "precipitously" along with the decrease of the delocalization length. These findings are consistent with those in Ref. 171. This study analytically clarifies the effects of (de)localizations of triplet and singlet excitons in SF. The authors also quantitatively analyzed the effect of the triplet-pair binding $(\chi)$ on the disentanglement of the triplets. Naturally, with a stronger binding, the triplets are more difficult to dissociate. 


\subsection{An Efficient Scheme to Locate SF-Favored Inter-Chromophore Conformations}

Calculating the SF-related transfer integrals is not very time consuming for one conformation. However, it becomes a formidable task if they need to be calculated millions of times in a numerical search for conformations that maximize the eg-tt effective coupling. Maximizing the coupling is not enough; the energies of the conformations shall not be too

high. Buchanan et al. developed a scheme for a quick conformation search. ${ }^{173}$ Their search function is defined as

$$
F=\alpha E_{r e p}^{2}-\left|H_{e g, t t}\right|^{2}
$$

$E_{\text {rep }}^{2}$ is the repulsion between the two monomers and can be approximated by atomic pairwise van der Waals force fields. $H_{e g, t t}$ is the effective coupling that includes both direct coupling and the CT-mediated coupling. The transfer integrals in the coupling are calculated using the Mulliken approximation and the Wolfsberg-Helmholtz formula. ${ }^{174,175}$ All these approximations significantly raise the efficiency in calculating the search function and its minimization. They enable $F$ evaluation for $10^{8}-10^{9}$ geometries in optimization of SF dimer conformation. This scheme was applied to locate the most SF-favored conformations for the models of ethylene dimer and cibalackrot (46) dimer.

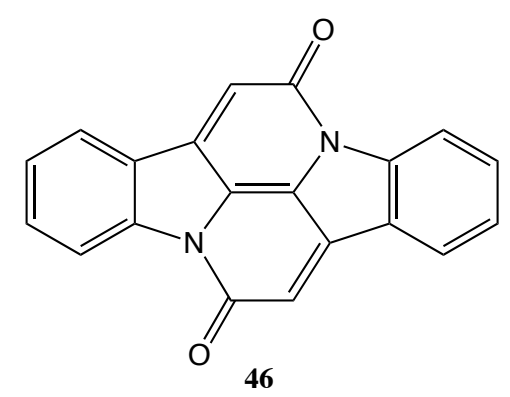

Following this study, Buchanan and Michl proposed packing guidelines for optimizing the effective $e g(g e)-t t$ couplings for chromophore dimers. ${ }^{176}$ The CT-mediated effective $e g$ - $t t$ coupling has the approximate form of

$$
\text { const } \times\left[S_{H H} S_{H L}-S_{L H} S_{L L}\right]=\text { const } \times\left[S_{P P} S_{Q P}-S_{P Q} S_{Q Q}\right] .
$$

$P$ and $Q$ stand for the orbitals of each monomer that are related to HOMO and LUMO as:

$$
P=\frac{1}{\sqrt{2}}(H+L) ; Q=\frac{1}{\sqrt{2}}(H-L) .
$$


The two sets of orbitals, $\mathrm{H}$ and L vs. $\mathrm{P}$ and $\mathrm{Q}$, give the same approximate expression in Eq. 20. Given evenly distributed HOMO and LUMO, P and Q are localized and more indicative for judging the magnitudes of inter-chromophore orbital overlaps and the effective coupling. On the other hand, typical polar chromophores have localized HOMO and LUMO, which are more indicative. The central idea for all packing guidelines is to maximize the

difference between $S_{P P} S_{Q P}$ and $S_{P Q} S_{Q Q}$, or between $S_{H H} S_{H L}$ and $S_{L H} S_{L L}$, so that the destructive interference is minimized. This conclusion is a generalization of what was found in a numerical study of an ethylene dimer model. ${ }^{177}$ Eq. 20 was derived assuming the same energies of the two CT states. Modulating the difference between the CT energies through changing packing geometries, introducing vacancy, or making acceptor/donor substitutions also provides viable ways to mitigate the destructive interference. ${ }^{109,113,157,158}$

\section{EXPLORATION FOR SINGLET FISSION MECHANISMS THROUGH DY- NAMICS SIMULATIONS}

In this section we first go over some studies that develop dynamics models for SF and provide fundamental understanding of the subject, and then some application studies.

\subsection{Simulations with Phenomenological Dissipation and Decoherence}

The first dynamics study for SF was published by Greyson et al. in $2010 .{ }^{178}$ Like in their earlier electronic study, ${ }^{109}$ the authors focused on covalently coupled dimers of SF chromophores. Their electronic Hamiltonian includes 10 Slater determinants, 8 of which give the $e g, g e, c a$, and $a c$ states, while the other two give the $(+1-1)$ and $(-1+1)$ components of $t t . \pm 1$ are the $M_{S}$ spin magnetic quantum numbers of the two triplets. Only the CT-mediated pathway was considered. $E(e g)$ and $E(t t)$ were approximated by vertical $E\left(S_{1}\right)$ and $2 E\left(T_{1}\right)$, and the CT energies were calculated using constrained DFT. The transfer integrals were calculated from orbital energy splittings as in Ref. 109 and following the Longuet-Higgins-Roberts approximation. ${ }^{179}$ Imaginary potentials were added to eg, ge, and $t t$ to simulate dissipation through those channels. With the dissipative electronic Hamiltonian, the coherent dynamics was simulated using the Liouville-von Neumann equation. The authors first investigated the SF dynamics with model Hamiltonians, for which the diabatic 
couplings and energies, and the dissipation potentials were tuned. The effects of these parameters on fission yields were isolated and elucidated. Small energy gaps among the CT states, eg, ge, and $t t$ were crucial for efficient coherent SF dynamics. The authors employed the same coherent model to calculate fission yields for 12 homodimers of pentacenes (3), 1,3-diphenylisobenzofurans (4), and polyenes. The results were in agreement with the understanding from the model simulations. The diabatic couplings were of less importance than the diabatic energies in affecting SF dynamics, and were considered primary factors for chromophore designing by the authors. The authors pointed out the limitation of their model (and thus the limitations of their conclusions), the lack of decoherence, which appears only when vibrational motions are considered (see Figure 3 and the relevant discussion above).

Chan et al. carried out a combined quantum chemistry and quantum dynamics study to explain the concurrent increases of the SE and ME signals in their time-resolved twophoton photoelectron (TR-2PPE) spectroscopic experiments of tetracene and pentacene. ${ }^{168}$ The concurrent increases made them believe that there is a coherent coupling between the two types of excitonic states. They employed the Multi-State Density Functional Theory (MSDFT) method to calculate couplings between SE, ME, and CT states for a cluster of 56 acenes with crystal packing geometry. The central 18 monomers were treated quantum mechanically and immersed in the electrostatic potential of the rest. The calculated couplings between the SE and ME states were as small as $0.5-3 \mathrm{meV}$, while the SE-CT and ME-CT ones ranged in 50-140 meV, indicating the CT-mediated nature of the SF in the acenes. The authors then simulated the SF dynamics in pentacene using the Liouville-von Neumann equation,

$$
i \hbar \frac{\partial \hat{\rho}}{\partial t}=\left[\hat{H}_{e l}, \hat{\rho}\right]-i \hbar \hat{D}
$$

$\hat{\rho}$ is the reduced density matrix of the electronic degree of freedom, $\hat{H}_{e l}$ the electronic Hamiltonian expanded in the SE, ME, and CT states, and $\hat{D}$ the phenomenological operator for decoherence. The MSDFT couplings and experimental diabatic energies were used to construct $\hat{H}_{e l}$. The authors simulated the SF dynamics with an evenly distributed SE state as the initial state for a dimer, a pentamer, and a decamer, which were all embedded in the crystal environment. The pentamer and decamer displayed similar SF dynamics, which was more efficient and featured more complete conversion to the ME states than in the dimer. The model's size is important: a large cluster supports SE delocalization, and a higher den- 
sity of ME states. The SF rate was sensitive to the decoherence rate. A fast decoherence prevents the reverse ME-to-SE conversion, i.e., the Rabi oscillation. The authors also employed the Redfield theory method as in Ref. 20 to simulate the SF dynamics with vibronic interaction. A reduced 3 diabats $(e g, t t$, and $c a$ ) model was used to describe the electronic system and an Ohmic spectral density (Eq. 28) was used to describe the vibronic coupling. The simulated SF dynamics was in good agreement with experiment.

\subsection{Development of System-Bath Models in SF}

In 2012, Teichen and Eaves developed very likely the first microscopic dynamics model of SF that includes both electronic and vibrational degrees of freedom. ${ }^{180}$ The vibronic coupling was discussed in the context of solvent-induced fluctuations. The model Hamiltonian reads

$$
\hat{H}=\hat{H}_{S}+\hat{H}_{B}+\hat{H}_{S B}
$$

It consists of the system part $\left(\hat{H}_{S}\right)$ of the 5 diabats, the bath part $\left(\hat{H}_{B}\right)$ of a bunch of harmonic oscillators (Eq. 3), and the interaction part $\left(\hat{H}_{S B}\right)$ that linearly couples the diabatic energies to the bath (Eq. 4 for diagonal elements with linear terms). This is a Holstein model, and the harmonic oscillators are used to simulate the solvent degrees of freedom, which induce fluctuations of the diabatic energies. The direct eg(ge)-tt couplings were set to be zero for their generally small magnitudes. The authors derived the formula for the conversion rate (a time-dependent non-Markovian rate) based on the quantum Liouville equation in the interaction picture, and further derived the approximate rate formula that has a form of Förster/Dexter exciton transfer rate:

$$
W_{j \rightarrow k}=\frac{J^{2}}{2 \pi} \int_{-\infty}^{\infty} d \omega A_{k}(\omega) I_{j}(\omega),
$$

where $J$ is the coupling between states $j$ and $k, A_{k}(\omega)$ and $I_{j}(\omega)$ the envelope functions related to the solvent-induced fluctuations of the states' energies. Assuming the state-solvent couplings to be described by the Debye spectral density (Eq. 27), the authors further derived the Fermi's golden rule rate formula

$$
W_{j \rightarrow k}=J^{2} \sqrt{\frac{\pi}{2 k_{B} T \gamma}} e^{-\frac{\left(E_{k}-E_{j}+2 \gamma\right)^{2}}{8 k_{B} T \gamma}},
$$

where $\gamma$ specifies the strength of the state-solvent coupling and $2 \gamma$ gives the solvent reorganization energy. The authors compared the non-Markovian and the golden rule rates that 
were calculated using parameters for typical SF problems and found that the former only converge to the latter when the time is longer than $600 \mathrm{fs}$, revealing the limitation of the golden rule formula. Due to the isomorphism between state-solvent and vibronic couplings, this model and the fundamental conclusions drawn from it are applicable to understand the effects of vibronic coupling in SF.

The first theoretical SF study that included both electronic structure calculation and quantum dynamic simulation was carried out by Berkelbach et al. ${ }^{20}$ The authors pointed out the importance of chromophore-localized diabats in understanding SF. Their characters are clear; their couplings to phonons can be calculated more easily than those of delocalized adiabatic states. Diabats are thus building blocks for a SF model. The authors gave a comprehensive introduction for electronic-phonon coupling, and various quantum master equations for the reduced density matrix of the electronic states. They especially focused on the Redfield theory. Using a 2 diabats system model with only eg and $t$ and an Ohmic bath (Eq. 28), the authors simulated the fission dynamics at the levels of time-local Redfield theory, secular and Markovian Redfield theory, and noninteracting blip approximation (NIBA). These results were compared with the numerical exact result obtained by the hierarchical equations of motion method. The electronic coupling and the system-bath coupling were varied to examine the applicabilities of the three levels of approximation. The authors also used the 3 diabats model, with both the sequential and the superexchange setting of energies, to examine the Redfield theory and NIBA. Both methods did very well in simulating the dynamics for the sequential model, while only the Redfield theory gave satisfactory results for the superexchange model. They also discussed how the eg-tt mixing in adiabatic states affects the absorption spectrum.

In the second paper of this series, ${ }^{181}$ Berkelbach et al. employed the Redfield theory with the secular and Markov approximations and the 5 diabats model to simulate SF dynamics for three pentacene dimers. The three dimers mimic the three symmetry-unique nearestneighbour pairs in pentacene crystal. The diabatic energies were taken as adjustable parameters, while the diabatic couplings were approximated by the transfer integrals (Eq 1). $E(e g)$ and $E(g e)$ were assumed to be identical, and so were $E(c a)$ and $E(a c)$. The authors scanned the relative energies $E(e g)-E(t t)$ and $E(c a)-E(t t)$ in the range of $0-1.5 \mathrm{eV}$ and simulated the SF dynamics for each point on the 2-D energy grid. $t t$ population as a function of the two relative energies and evolution time was examined. CT states with their 
energies about $1.5 \mathrm{eV}$ higher than the SE states could still mediate efficient SF. The SF efficiency is more dependent on $E(e g)-E(t t)$ than on $E(c a)-E(t t)$. This was consistent with the Marcus theory's rate constant formula, with the effective eg-tt coupling estimated using the perturbation theory. The difference between the superexchange and the sequential mechanism in SF dynamics was clearly demonstrated in Figure 13: while the low-lying CT states lead to more efficient SF in the sequential model, the eventual ME population is lower due to the more substantial CT population. The authors elucidated the limitations of the Föster theory and NIBA: their master equations are perturbative to second order in the electronic coupling, while the superexchange mechanism involves fourth-order coupling; the two methods therefore can only describe sequential SFs, not the superexchange analogues. The authors also pointed out another key factor to raise SF efficiency: having phonons whose frequencies are in resonance with the SE-ME gap.
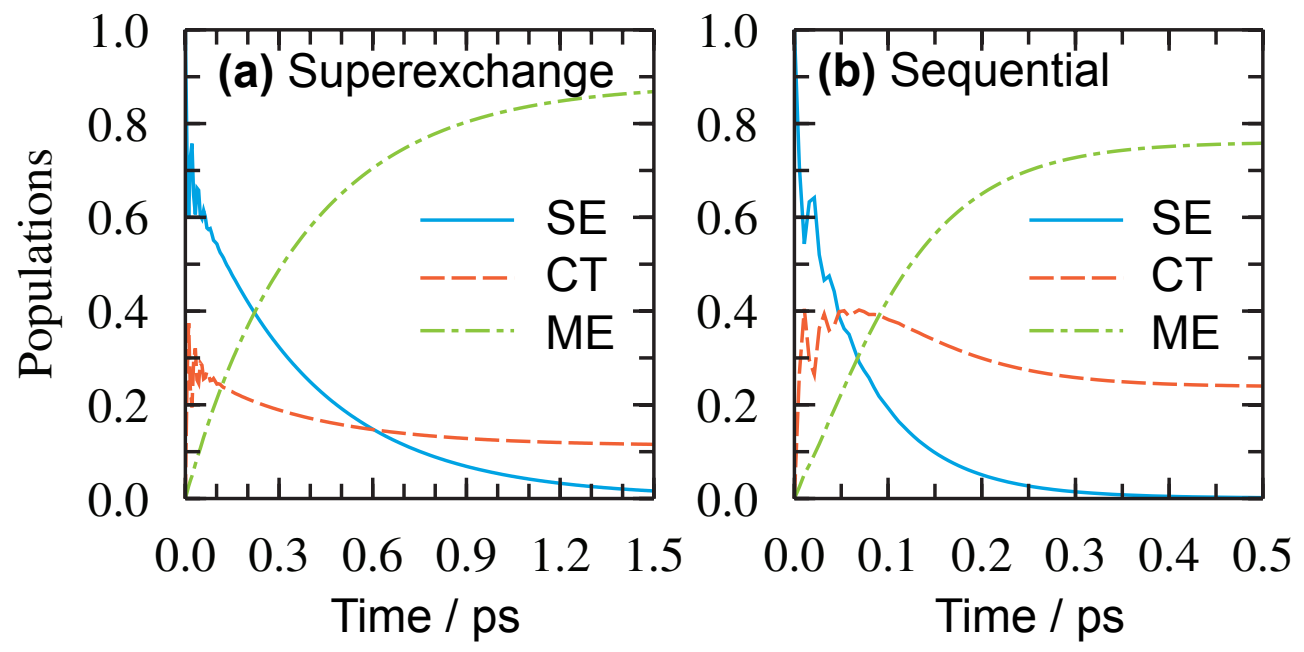

Figure 13. Simulated SF dynamics with typical superexchange (a) and sequential (b) settings of diabatic energies. This figure is adapted with permission from Ref. 181; Copyright 2013, American Institute of Physics.

In the third and last paper of this series, ${ }^{139}$ Berkelbach et al. used the same method to investigate SF in crystalline pentacene. Their crystal model only allows for nearest-neighbour interaction. The HOMO-to-LUMO gap and the transfer integrals were renormalized from gas phase values to incorporate screening effects in solid. Clusters with 10, 27, and 52 molecules on the $a-b$ plane of pentacene crystal were studied to ensure convergence of simulated results towards the crystal limit. The modelled band structure is in good agreement 
with the $a b$ initio result. ${ }^{182}$ The simulated spectra of crystalline pentacene are in satisfactory agreement with experiment. The lowest bright state was found to contain half SE and half CT characters. The Redfield theory simulation gave a $270 \mathrm{fs}$ SF time scale, which is qualitatively similar to the $80-200$ fs experimental value. The substantial CT character in the lowest bright state facilitates the SE-to-ME transition. This series of three papers gives an overall perspective of SF and presents rich fundamental knowledge of this subject.

\subsection{Including Vibrations in the System Hamiltonian}

The importance of vibronic coupling in SF has been further manifested in recent experiments. Vibronic couplings do not just dissipate the excessive energy in SF, modulate the coupling strength between chromophores, and lead to decoherence between SE and ME states. Furthermore, SF may proceed through a conical intersection between SE and ME states, ${ }^{183} \mathrm{ME}$ vibronic state may be in resonance with the initial SE state, ${ }^{184}$ and there may be coherent coupling between SE and vibronically excited ME state. ${ }^{185}$ All these vibronic effects facilitate SF. Motivated by these findings, Tempelaar and Reichman constructed a vibronic model to simulate SF in crystalline pentacene. ${ }^{38}$ It is a solid state model that satisfies periodic boundary conditions. The electronic part of the system Hamiltonian is similar to the one in Ref. 139. One vibrational mode in each molecule with the frequency of the symmetric stretching of pentacene is included in the system. Each diabat is linearly coupled to the vibrations of the molecules that are excited or ionized in the diabat. Pure vibrational excitation in the ground state of a molecule is allowed. The vibrational degrees of freedom are not treated as a continuous phonon bath as in Ref. 139. Their interactions with the electronic states are treated non-perturbatively, with justified state-specific coupling constants. Through comparing the simulated and experimental absorption spectra, the authors determined the diabatic energies, and found that the smallest model unit cell that gives converged results contains $3 \times 3$ pentacene unit cells in the $a b$-plane. Through analyzing the distance correlation between triplet excitons in $t$-dominated states, the authors concluded that the triplet-pair mainly resides at adjacent molecules.

Tempelaar and Reichman further improved their model by including vibrational excitations in the ground electronic state and expanding the ME space to include triplet-pair with one $T_{1}$ and one $T_{n>1}$ excited states. ${ }^{186}$ The authors used this full model to simulate the two- 
dimensional electronic spectroscopy (2DES) of crystalline pentacene, ${ }^{186}$ the first experiment that gave direct detection of the $t t$-type state. ${ }^{184}$ This is the first simulated 2DES in the context of SF, and its agreement with the experimental spectrum is satisfactory. Through analyzing the simulated spectrum, the authors concluded that the probed excitation occurs between $T_{1}$ and $T_{2}$, and estimated its transition dipole moment to be 8 to 15 times of that of the $S_{0}$-to- $S_{1}$ excitation. The vibronic couplings of $T_{1}$ and $T_{2}$ and the $T_{2}$ delocalization were also found to be important in simulating 2DES.

\subsection{Application Studies}

\subsubsection{Surface hopping simulations}

Mou et al. carried out the first theoretical simulations for SF in amorphous systems. ${ }^{187}$ They prepared a simulation box with 128 5,12-diphenyltetracene (25) molecules and their amorphous structures were obtained by the melt-quench procedure in molecular dynamics. Periodic boundary conditions were imposed. The authors simulated the SF process in this large system in a divide-conquer-recombine fashion. The HOMO-to-LUMO singlet excited state on one molecule, described using TDDFT, is allowed to hop to the two nearest neighbours and this nonadiabatic process was simulated at the level of surface hopping. ${ }^{188}$ Most of the time, the singlet exciton was found to be monomer-localized. During the hopping, the SE-to-ME fission was simulated using Fermi's golden rule. These were the divide-conquer steps. With the calculated hopping and fission rates, the authors performed kinetic MonteCarlo simulations in the whole system (recombine). The simulated SF dynamics was in good agreement with experiment and captured the two SF time scales of $\sim 1$ and $\sim 100$ ps. ${ }^{53}$ Consistent with the hypothesis given in an experimental study, ${ }^{53}$ the short time scale corresponds to fast SF at some "hot spots" and the long time scale corresponds to the diffusion of excitons to those spots. The geometrical features of the hot spots dimers were revealed.

Akimov and Prezhdo simulated the SF and charge transfer dynamics at the pentacene $/ \mathrm{C}_{60}$ interface. ${ }^{189}$ They imposed periodic boundary conditions in simulating the interface. Each unit cell contained two pentacene molecules and one $\mathrm{C}_{60}$, which was used as the acceptor

in the first SF-based OPV cell. ${ }^{12}$ Their nonadiabatic dynamics simulation was based on the fewest switches surface hopping (FSSH) method, ${ }^{188}$ and their electronic diabats were 
constructed by distributing four electrons in the frontier orbitals: HOMO and LUMO of the pentacenes, the 3-fold degenerate LUMO and non-degenerate LUMO+1 of $\mathrm{C}_{60}$. The simulation showed the SE state underwent a quick charge transfer to $\mathrm{C}_{60}$, instead of fissioning to a triplet-pair on two pentacenes. We believe that the SF efficiency may have been underestimated: $c a$ and $a c$ in Figure 2 were not included in the model and therefore the CTmediated SE-to-ME transition was absent. Following the conclusion in Ref. 112, the authors included the intra-pentacene multi-excitonic states in their model. However, as discussed above, this state has too high energy to participate in pentacene SF. ${ }^{113}$

Wang et al. applied the self-consistent FSSH method ${ }^{190}$ to investigate the relation between SF efficiency and intermolecular packing for a pentacene dimer model. ${ }^{191}$ The 5 diabats model and the Holstein vibronic Hamiltonian with one effective vibrational mode coupled to each state were used. The transfer integrals that determine the diabatic couplings, CT character of the initial bright state, instantaneous and eventual tt yields, and SF time scale were calculated along the longitudinal and transverse relative motions of the two monomers, which were vertically displaced by $3.4 \AA$ (Figure 14). Through analyzing the correlations among these quantities, the authors found that although the CT-mediated couplings are necessary for SF, large CT character in the initial SE-dominated state may also lead to excimer formation and hinders SF. The instantaneous $t t$ population through its mixing with $e g$ and ge correlated well with the SF rate. The authors also found that the thermal fluctuations of the diabatic energies alleviate the symmetry selection rules on the diabatic couplings. The simulated SF rate is consistent with the prediction of the Yost kinetics model, ${ }^{126}$ that as the eg(ge)-tt couplings are larger than a threshold, the rate reaches a plateau.

\subsubsection{Symmetrical quasi-classical simulations}

Tao applied the newly developed symmetrical quasi-classical (SQC) method to study SF in pentacene dimer models. ${ }^{192}$ In contrast to the Redfield theory, this method treats both the electronic and nuclear degrees of freedom explicitly and at the same dynamical footing. Both the 3 and 5 diabats models were examined. The simulated results were similar to those obtained using the Redfield theory. ${ }^{181}$ Despite the small direct couplings, Tao's simulations showed an interference between the direct and the CT pathways, which noticeably affects the short-time dynamics. The direct couplings may not be ignored. 


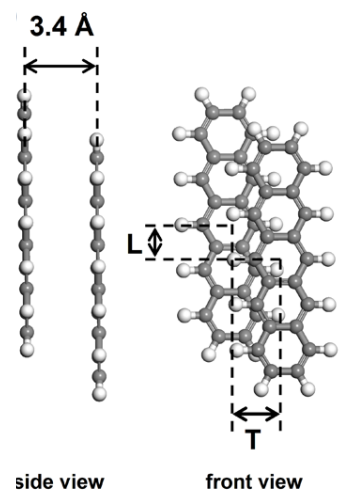

Figure 14. The pentacene model dimer used in Ref. 191. L and T stand for the longitudinal and transverse directions of the relative motion between the monomers. This figure is taken with permission from Ref. 191; Copyright 2014, American Chemical Society.

Using the same method and the 3 diabats superexchange pentacene dimer model, Tao investigated the bath effect in SF dynamics. ${ }^{193}$ Four types of bath were examined: Debye, Ohmic, pseudo local, and a single frequency one. Spectral density is defined as

$$
J(\omega)=\frac{\pi}{2} \sum_{k} \frac{c_{k}^{2}}{\omega_{k}} \delta\left(\omega-\omega_{k}\right),
$$

where $\omega_{k}$ is the angular frequency of the $k$-th bath mode, and $c_{k}$ the coupling strength of the mode to an electronic matrix element. Keeping only one mode in the summation gives the single frequency bath. The other three baths correspond to different broadening approximations: Debye:

$$
J(\omega)=\frac{2 \lambda \omega \omega_{c}}{\omega^{2}+\omega_{c}^{2}}
$$

where $\omega_{c}$ is the cut-off frequency and $\lambda$ the total reorganization energy; Ohmic:

$$
J(\omega)=\eta \omega e^{-\omega / \omega_{c}},
$$

where $\eta$ represents the coupling strength and the total reorganization energy is $\eta \hbar \omega_{c}$; pseudo local:

$$
J(\omega)=\sum_{k}^{N_{k}} \frac{\lambda_{k} \omega \Gamma_{k}}{\left(\omega-\Omega_{k}\right)^{2}+\Gamma_{k}^{2}},
$$


where $\lambda_{k}$ is the reorganization energy of constituent spectral density centered at frequency $\Omega_{k}$ and $\Gamma_{k}$ the Lorentzian broadening parameter.

The Debye, Ohmic, and pseudo local baths were shown to give similar SF dynamics, while the single frequency bath gives much slower SF. Debye and Ohmic baths with low characteristic frequency of $5 \mathrm{meV}$ led to slower yet not too slow SF. The effect of reorganization energy was studied using the Debye bath. An optimal value of $100 \mathrm{meV}$ was found. Baths with high characteristic frequencies gave temperature-independent SF dynamics in the range of $T=100-300 \mathrm{~K}$; the bath modes are not activated at these temperatures. Temperature dependence was observed for simulations using baths with low characteristic frequencies. A bath mixed with components of low and high characteristic frequencies may be needed to describe the temperature dependence of SF. Noticeable interference between the direct and CT-mediated couplings was seen in both baths with high and low characteristic frequencies.

Tao also studied the effects of diabatic energies, diabatic couplings, and reorganization energies on SF using the 3 diabats pentacene dimer model. ${ }^{194}$ The diabatic energies were varied to cover the five situations: normal superexchange, superexchange with high CT energy, sequential, normal superexchange with smaller exoergicity in SF, and normal superexchange with endoergicity in SF. SF rates were estimated at the levels of SQC, the Föster theory, and the Marcus theory. The SQC results were treated as benchmarks. The effectivenesses and limitations of the Föster theory and the Marcus theory were discussed.

\subsubsection{SF dynamics of perylenediimide}

Renaud et al. investigated the influence of stacking geometries on $\mathrm{SF}$ in perylenediimide (47 PDI) dimer. ${ }^{195}$ Their kinetic SF model consists of three states: $e g, c a$, and $t t$. The SF was described as a superposition of the two kinetic channels:

$$
e g \rightarrow t t ; e g \rightleftharpoons c a \rightarrow t t
$$

The rate constants were calculated using the Marcus theory, with the couplings being evaluated using the transfer integrals, and the Gibbs free energies and reorganization energies being calculated at (TD)DFT level. In this model, $c a$ is treated as a real intermediate; only when it is substantially populated the CT-mediation plays a role. Since $c a$ lied more than 2 
$\mathrm{eV}$ above $e g$, the $e g$-to-ca transition was highly unfavorable, and so was the CT-mediation in the SF. The eg-tt coupling and the consequent SF efficiency were sensitive to the stacking geometry of the dimer. The authors identified the favorable geometries that gave optimal SF yields by scanning the stacking geometries of two PDI molecules. Through comparing the theoretically derived favorable geometries and experimental structures of PDI derivatives, the authors identified promising derivatives as SF chromophores. No dynamics simulations were carried out in this study. Due to its close connection with the two following works, it is placed in this section.

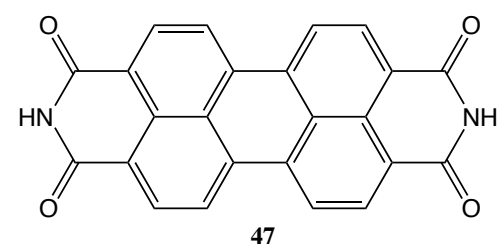

Mirjani et al. employed the Redfield theory to simulate SF dynamics in molecular dimers of pentacene (3), DPB (4), and PDI (47). ${ }^{196}$ They investigated how the stacking geometry affects the SF rate and the competition between the direct and the CT-mediated pathways. The dimers were described using the 5 diabats model. $E(e g)$ and $E(g e)$ were approximated by the monomeric $E\left(S_{1}\right)$, and $E(t t)$ by $2 E\left(T_{1}\right) . E(c a)$ and $E(a c)$ were calculated based on the direct reaction field (DRF) method, with the dimers being embedded in their respective crystalline environments of polarizable molecules. The monomeric excitation energies were calculated at TDDFT level. The Holstein couplings were described using an Ohmic bath. The authors calculated four types of reorganization energies: of HOMO, LUMO, $S_{1}$, and $T_{1}$. The vibrational modes that gave the largest reorganization energies were concentrated around the frequency of $0.15 \mathrm{eV}$, corresponding to $\mathrm{C}-\mathrm{C}$ stretching and being set as $\omega_{c}$. The summed reorganization energies of the four types were averaged for each monomer, and then averaged over the monomers to give the $0.135 \mathrm{eV}$ reorganization energy for the dimer model bath. The simulations again demonstrated the importance of stacking geometry. The four PDI dimers that differ in stacking have their SF rate constants ranging from $0.56 \mathrm{~ns}^{-1}$ to $79.0 \mathrm{ps}^{-1}$. Crystal structure engineering is hence a handle to enhance SF efficiency. Different from the finding in Ref. 195, the CT-mediated pathway dominated the SF processes, and there was an interference between the CT-mediated and the direct pathways.

In crystal, the stacking geometry is modified by low-frequency intermolecular vibrational modes. The importance of stacking geometry naturally indicates the importance of inter- 
molecular vibrational modes on SF. Renaud and Grozema employed a PDI dimer model to investigate the effects of intermolecular vibrations on SF, specifically through the Peierls couplings. ${ }^{197}$ They solved the master equation for the reduced density matrix of the 5 diabats using a non-Markovian quantum jump approach. The CT energy relative to $E$ (eg) was varied so that both superexchange and sequential mediations were examined. The Peierls couplings affected the direct pathway and facilitated efficient SF in the superexchange region. The authors then constructed an infinite one-dimensional chain model with identical molecules for four specific PDI species, and simulated the effects of phonons of intermolecular vibration characters on their SF dynamics. Three species with the two-electron integrals being large were affected by the Peierls couplings. This study indicates that even when CT states are too high in energy to mediate the effective couplings, SF may still occur through the direct pathway under the assistance of intermolecular vibrations.

\subsubsection{Effects of surface polarity on SF dynamics}

In a dye-sensitized solar cell, SF is a competitive process only when a spacer layer slows down electron injection of singlet excitons. ${ }^{198}$ This finding motivated Strong and Eaves to simulate the aggregation of tetracene on a model surface and investigate the dependence of the packing geometry of the aggregate on the polarity of the surface. ${ }^{199}$ The surface was modelled as a slab of point dipoles. When the dipoles are aligned, the surface exhibits macroscopic polarity; when they are randomly oriented, the surface exhibits microscopic polarity; when the dipoles are set to be zero, a nonpolar surface is obtained. Tetracene molecules adsorbed on the surface were simulated using the DERIDING force field and the replica exchange molecular dynamics. Despite the low concentration of tetracene molecules in the simulation, they tended to self-assemble and form herringbone aggregates, which promote SF. A microscopically polar surface provides inhomogeneous potential for the tetracenes and tend to trap them in local minima of the potential. This hinders the tetracene aggregation and the formation of herringbone clusters. The inhomogeneity disappears in a macroscopically polar surface, which facilitates the aggregation formation. Therefore, the surface polarity plays a subtle role in promoting SF. The authors concluded that the detailed surface structure matters, not just the polarity of the surface molecules. 


\subsubsection{Multi-configuration time-dependent Hartree (MCTDH) simulations}

Tamura et al. employed the MCTDH method to study the coherent SF in TIPS-pentacene (the monomer in 31) crystal and the non-coherent, thermally activated SF in rubrene (26) crystal. ${ }^{41}$ Each of the crystals was approximated by a trimer with crystal packing geometry. The 5 diabats model for dimer was extended to the 9 diabats model for trimer: 3 SE states, 2 ME states with the triplet-pair residing on adjacent monomers, and $4 \mathrm{CT}$ states that involve charge transfer between adjacent monomers. The vibronic model included both Holstein and Peierls couplings. Both the CT-mediated and the direct eg(ge)-tt couplings contributed to the $\sim 100 \mathrm{fs}$ SF in TIPS-pentacene, and the SF dynamics was independent of whether the initial SE state is localized or delocalized, bright or dark. The substantial overlap between the vibrational wave packets on the SE and ME states confirmed the experimentally observed vibronic coherence in the efficient SF. ${ }^{183}$ The authors attributed the coherence to the proximity of the SE-ME crossing to the Franck-Condon region, and the large CT-mediated effective couplings. On the contrary, the effective couplings are symmetry-nullified at the undistorted structure of the rubrene trimer. They were turned on by intermolecular symmetry-breaking modes, whose thermal excitations are essential for the SF in rubrene. This study clearly explains the difference between SFs in rubrene and in tetracene. Although both are endoergic, the latter is temperature independent. ${ }^{200}$ Tetracene's herringbone packing allows for effective SE-ME couplings; thermal excitation of symmetry-breaking modes is not needed.

Zheng et al. employed the multi-layer MCTDH method to simulate the SF dynamics of a 3 diabats pentacene dimer model, ${ }^{201}$ with all states being Holstein-coupled to identical, independent Debye baths (Eq. 27). The simulated dynamics was in good agreement with the results obtained using the Redfield theory, HEOM, and SQC methods, demonstrating the robustness of MCTDH in simulating SF dynamics. The authors ordered the vibrational modes into eight groups according to their frequencies, and performed eight simulations. The vibrations that have their frequencies in resonance with the eg-tt gap played the most significant role in driving the eg-to-tt conversion, in consistence with the findings in Refs. 181 and 197. Some of the other modes participate in SF by inducing decoherence between the diabats. The authors clarified the importance of both the resonance and the vibrational overlap of vibronic states of the $e g$ and $t t$ diabats (Figure 15). The resultant degenerate and overlapping vibronic levels of the SE and ME manifolds facilitate efficient conversion from 
the former to the latter. The authors concluded that an ideal spectral density for SF should have substantial amplitude at the frequency that match the eg-tt gap. The large amplitude indicates significant vibronic couplings of the two states (or just one) to the vibrational modes, hence large displacement of the equilibrium positions of the vibrational modes on the two diabats, and hence large vibrational overlaps.

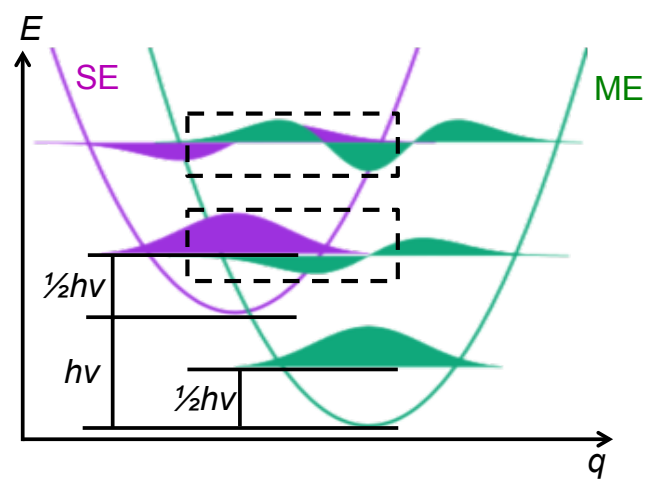

Figure 15. SE and ME potential energy curves along a vibrational coordinate $q$. The vibrational frequency is in resonance with the SE-ME energy gap. The rectangles highlight the degeneracies and good overlaps between vibrational levels of the two diabats.

Inspired by a report of high yield iSF in quinoidal bithiophene (QOT2, 48), ${ }^{202}$ Chien et al. investigated the excited states dynamics of this species. ${ }^{203}$ They calculated the low-lying excited states' potential energy surfaces using the XMS-CASPT2 method and found a conical intersection between the lowest bright ${ }^{1} B_{u}$ and the lowest dark ${ }^{1} A_{g}$ excited state close to the Franck-Condon region. This proximity results in a fast ${ }^{1} B_{u}$-to- ${ }^{1} A_{g}$ transition. The transition was simulated using the MCTDH method. The simulation included 3 tuning modes, 1 coupling mode, and 20 bath modes that mimic an Ohmic bath (Eq. 28) and dissipate the released energy. The $76 \mathrm{fs}$ simulated transition time scale is in qualitative agreement with the experimental finding. ${ }^{204}$ The ${ }^{1} A_{g}$ state contained $75 \%$ triplet-pair character, and its mixing with the other $25 \%$ component resulted in a substantial stabilization. The triplet-pair is hence strongly bound. The authors found a $1.76 \mathrm{eV}$ barrier to reach a twisted structure that gives the uncoupled triplets. It is thus difficult to harvest the triplets in QOT2's iSF.

Quinoidal tetrathiophenes (QOTT, one of this class of structures, QOTT- $(\mathrm{CN})_{2}-\left(\mathrm{CO}_{2} \mathrm{Me}\right)_{2}$, is shown in 49) share similar structures with QOT2. However, the QOTT species were found to be SF inactive. ${ }^{205}$ To understand this difference, Momenti employed the CASPT2 method 

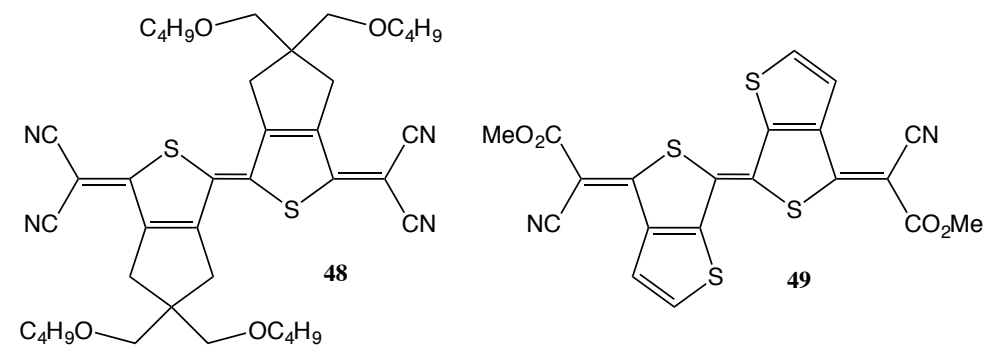

to investigate the low-lying excited states of a series of QOT2 and QOTT structures. ${ }^{206} \mathrm{He}$ found the SE-ME crossings to lie higher in energy and far away from the Franck-Condon region for the QOTT structures. This explains the absence of SF in QOTT. Dynamic correlation was found to substantially modify excitation energies and crossings of potential energy surfaces. Although this is an electronic structure study, it is placed in this section due to its close connection to Ref. 203

Zeng and Goel used MCTDH to simulate iSF dynamics for dimers of small chromophores. ${ }^{207}$ The motivation was to design small iSF chromophores. The authors attempted to design homodimers of the three azaborine chromophores proposed in Ref. 96 (11-13). Since B and N provide the important captodative effect, they were not considered as connecting sites. The authors examined the HOMO and LUMO amplitudes at all $\mathrm{C}$ sites and found the largest difference between them in one $\mathrm{C}$ site in the BN-azulene (13). The large amplitude difference implies a large difference between the parenthesized terms in the CT-mediated eg(ge)-tt couplings,

$$
\frac{t_{H L}\left(t_{L L}-t_{H H}\right)}{\Delta E_{C T}}
$$

and hence a small destructive interference. The authors then constructed the corresponding dimer models, 50-52, and examined their SF dynamics. The simulated iSF in $\mathbf{5 0}$ was completed in 1.5 ps. However, the eventual $t t$ population was only $68 \%$ due to its substantial mixing with CT states. The mixing is a manifestation of the strong binding between the two triplets, ${ }^{35}$ and arises from the large inter-chromophore $\pi-\pi$ overlaps. To reduce the mixing, the authors proposed to methylate on the $\mathrm{N}$ in the 5 -membered ring so that the steric hindrance increased the dihedral angle from $35^{\circ}$ to $64^{\circ}(\mathbf{5 1})$. However, since the monomeric LUMO (HOMO) has (has no) amplitude at the $\mathrm{N}$ site, its energy is (is not) raised by the weak $\pi$ donor methyl. Consequently the monomeric HOMO-LUMO gaps is enlarged, and $E(t t)$ is raised. The resultant smaller energy distances between $t t$ and CT states cancelled the reduction of their coupling matrix elements. The $t t$ population was only increased to 
76\%. The authors then changed to methylate on a $\mathrm{C}$ site as shown in $\mathbf{5 2}$. The dihedral angle was increased to $76^{\circ}$. Both the monomeric HOMO and LUMO have amplitudes at this site, and their energies were both increased and the HOMO-LUMO gap was largely invariant. The energy distances between $t t$ and CT states were similar to those in 50. Therefore, the larger dihedral angle reduced the $t t$-CT couplings as planned. The simulated SF of $\mathbf{5 2}$ was completed in 1 ps and the eventual tt population was increased to $90 \%$. 52 is hence the most promising intramolecular SF chromophore designed in this work. The general small size effects on intramolecular SF were thoroughly discussed in this paper. The CT states are doomed to be low-lying due to the short distance between the separated charges. The frontier orbitals are concentrated due to the small chromophore size and hence their amplitudes at the connecting sites are large, and so are their overlaps. These two factors result in generally strong $t t$-CT mixing. In reducing the mixing through steric hindrance, the substitution site for the steric group needs to be carefully selected as the substituent may affect the electronic structure unexpectedly, like in $\mathbf{5 1}$
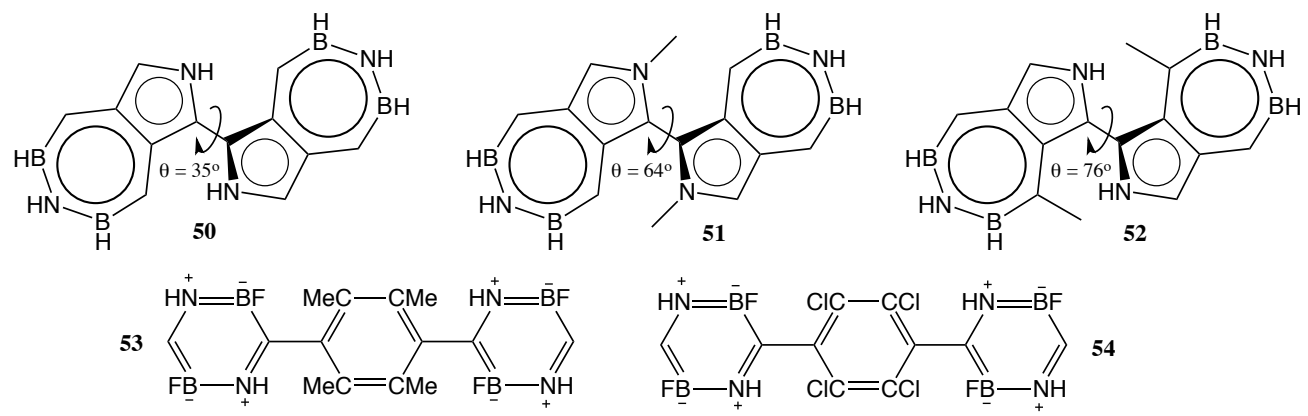

In another paper, Zeng studied the mechanism of through-linker iSF using a model chromophore, ${ }^{208}$ in which two units of $\mathbf{1 1}$ were covalently connected to a tetramethyl-paraphenylene (53). The methyls were introduced to induce large dihedral angles between the chromophore units and the linker and thus a weak through-linker triplet-pair binding. The author elucidated the electronic coupling pathway for the through-linker iSF as shown in Figure 16. The conversion from the $\mathrm{SE}(E G G)$ to $\mathrm{ME}(T G T)$ state is realized by a series of one-electron hoppings, which involve charge-resonance configurations between the chromophore unit and the linker (e.g., the $C R G$ diabat) and configurations that have both triplet-pair and $\mathrm{CT}$ characters (e.g., the $(c a)^{t} t$ configuration in the $C G A$ diabat). Within the coupling pathway there are two branches, passing through the cag and the $a c g$ configurations in the $C R G$ diabat, respectively. The two branches interfere destructively. In 53, 


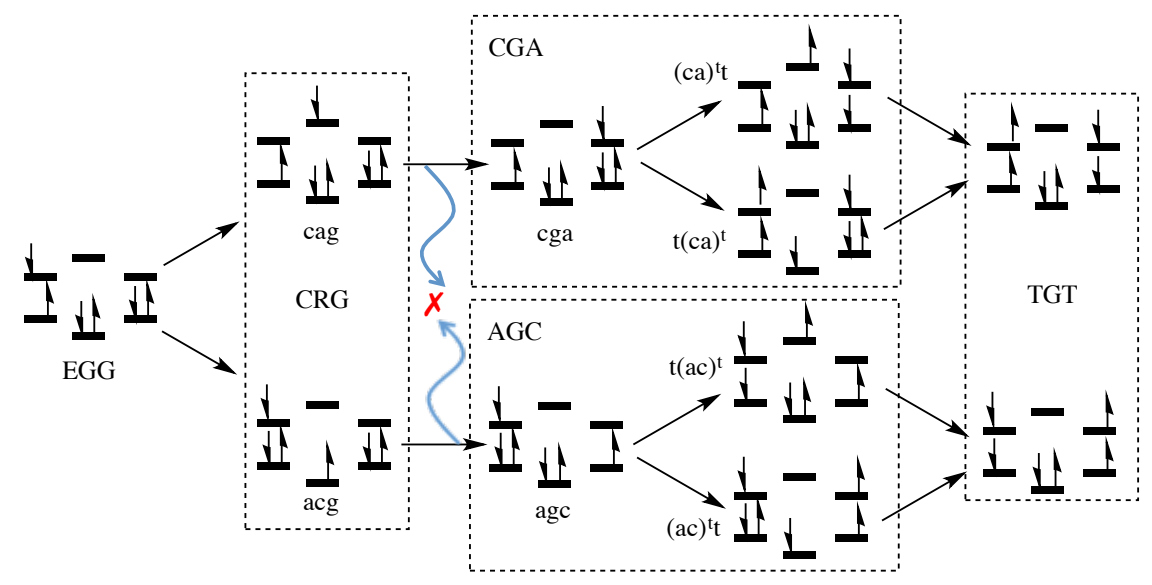

Figure 16. Electronic coupling pathway for the through-linker iSF in 53. The upper case symbols label diabatic states, while the lower case label the electronic configurations contained in the diabats. The blue curved arrows and the red cross illustrate the destructive interference between the two branches in the coupling pathway.

cag and acg contribute almost equivalently to $C R G$, maximizing the interference. MCTDH simulation showed that the iSF was completed in $12 \mathrm{ps}$, with a $70 \%$ eventual ME population. To improve the performance, the author proposed to replace the four methyls by four $\mathrm{Cl}$ atoms (54). The electronegative $\mathrm{Cl}$ atoms pulled down the HOMO and LUMO energies of the linker so that the cag configuration is of lower energy than the acg configuration; only the former contributed to the $C R G$ diabat, and only the upper branch in Figure 16 was effective. With the interference being removed, MCTDH simulation showed a 3 ps iSF and a 90\% eventual ME population. The key contributions of this work are the elucidation of the electronic coupling pathway in this type of through-linker SF and the strategy to enhance iSF efficiency by making/changing substitutions on the linker.

\subsubsection{A SF dynamics study for polyenes using electronic Hamiltonian}

Prodhan and Ramasesha conducted a quantum dynamics study on SF in polyenes. ${ }^{209}$ The polyenes include 1,3-butadiene, 1,3,5-hexatriene, and 1,3,5,7-octatetraene. Each polyene adopts two dimer conformations, the vertical and the horizontal stackings. The authors treated the dimers using the PPP model and the Hubbard model. The initial state was propagated using the electronic Hamiltonian, and the projection of the evolved state onto 
the triplet-pair state was examined. Two monomeric initial states were considered, the bright $1^{1} B$ state and the dark $2^{1} A$ state. The authors found for all the dimers that the $2^{1} A$ state and the triplet-pair state have projections on the same eigenstates of the Hamiltonian, while the $1^{1} B$ state and the triplet-pair state are never mixed. They concluded that SF in polyenes only occurs with the $2^{1} A$ state initial state. The authors also found that when the polyenes are substituted with donor and acceptor groups, the $2^{1} A$ state loses triplet-pair character and the fission yield is significantly reduced. This seems inconsistent with the finding of Ren et al. for PBTDO. ${ }^{153}$ Further studies are needed to reconcile the two works.

\subsubsection{A second-order time-convolutionless quantum master equation study for pentacene}

Nakano et al. studied the SF dynamics of a pentacene dimer model. ${ }^{210}$ The pentacene model assumed symmetries between $e g$ and $g e$, and between $c a$ and $a c$. The relative energies of $t t, e g$, and $c a$ were tunable parameters. The system-bath interaction was approximated by Ohmic spectral densities (Eq. 28). The evolution of the reduced density matrix of the 5 diabats was described using the second-order time-convolutionless quantum master equation. Through analyzing the relative relaxation factors in the equation, the authors qualitatively explained the features of SF dynamics with different parameters. The eg-ge coupling modified the energies of the symmetric and antisymmetric combinations of $e g$ and $g e$ and their mixings with the CT states, and also the SF dynamics. The authors also pointed out that the near degeneracy between the SE- and $t$-dominated adiabats may lead to fast SF but low $t t$ yield, due to the significant eg(ge)-tt mixing. When the more realistic state-dependent baths were used, both fast SF and high $t t$ yield can be obtained. The authors emphasized the importance of the vibronic coupling of the CT states in giving the fast and complete SF.

\subsubsection{Diabats coupled to the same vibrational mode}

In most of the SF vibronic dynamics simulations, diabats were assumed to be coupled to independent phonon baths. Fujihashi and Ishizaki developed a model that contains 3 diabats, $e g, c a$, and $t t$, and they were coupled to one reaction (vibrational) coordinate but with different reorganization energies. ${ }^{211}$ The relaxation function of the vibrational coordinate was approximated by a single exponential function. The $c a-e g$ and $c a-t t$ energy gaps were 
functions of the coordinate and fluctuated in time. The signs of the vibronic coupling constants were also treated as adjustable parameters to investigate the correlations between the diabats in vibronic coupling. The central result is that the fluctuations in the energy gaps do not impede the CT-mediated SF. The reorganization energies and vibronic correlations affect the coherent eg-tt oscillation, but not the overall behaviour of the SF dynamics.

\subsubsection{The importance of a vibronic system Hamiltonian in tetracene SF}

Morrison and Herbert employed their recently developed ab initio Frenkel-Davydov exciton model (AIFDEM) to construct the 5 diabats model for the crystal unit cell tetracene dimer. ${ }^{212}$ The mixing between $e g(g e)$ and $t t$ was not substantial in the adiabats, indicating that the SF in tetracene crystal does not occur following an electronic coherence mechanism. The authors calculated the nonadiabatic matrix elements between the eigenstates and transformed them to the diabatic vibronic coupling parameters. The vibronic couplings of the diabatic energies were substantial along four $\mathrm{C}-\mathrm{C}$ stretching modes with frequencies from 1432 to $1540 \mathrm{~cm}^{-1}$, while all off-diagonal vibronic couplings were small. This justified the use of a Holstein model. Those frequencies are in resonance with the eg(ge)-tt gap; the resultant resonance between $e g(g e)$ and $t t$ vibronic states leads to efficient conversion from the former to the latter (Figure 15). Considering only the mode with the most significant vibronic coupling, the authors diagonalized the vibronic Hamiltonian. The resultant vibronic eigenstates exhibited significant mixing between $e g(g e)$ and $t t$ vibronic states. This suggests a vibronic coherence mechanism for the tetracene SF. The authors also simulated the SF dynamics using the Redfield theory and an Ohmic bath (Eq. 28), with the electronic and vibronic system Hamiltonians, respectively. Only when the vibronic system Hamiltonian was used the simulated dynamics was in consistence with the experimental observation: a simultaneous rise-up of $t t$ population after photo-excitation. ${ }^{168}$

\subsubsection{SF induced by Peierls coupling}

Huang et al. studied the role of Peierls coupling in SF using a model that contains

one SE and one ME state. ${ }^{213}$ Both Holstein and Peierls couplings were described using the underdamped Brownian oscillators spectral densities. The SF dynamics was simulated using 
the multi- $D_{2}$-Ansatz. The Peierls couplings were found to be responsible for SF when the electronic coupling between the SE and ME states is weak. This is a reasonable result; if the electronic coupling at the reference geometry is weak, we may only rely on intermolecular vibrational motion to modulate and enhance the coupling.

Castellanos and Huo investigated the destructive interference between the two branches in the CT-mediated coupling. ${ }^{214}$ They employed the Partial-Linearized Density Matrix PathIntegral method to simulate the SF dynamics of a pentacene dimer with the 5 diabats model. Both Holstein and Peierls couplings were included and described using Debye spectral densities (Eq. 27). The most interesting finding, we think, is that the destructive interference can be alleviated by the fluctuations of the couplings between the CT states and $t t$, which arise from the relevant Peierls couplings to intermolecular vibrations.

\subsubsection{J- or H-type coupling of SE}

Most recently, Zang et al. studied the effects of the $J$ - and $H$-aggregates on quantum interference in SF using a 5 diabats pentacene dimer model. ${ }^{215}$ They focused on the influence of the eg-ge effective coupling, $\tilde{J}$, which determines whether their coupling is of $J$ - or $H$ type. In their model, $\tilde{J}$ has contained the contributions from the higher-lying CT states in mediating the coupling between the two SE states. They used $\tilde{V}_{1}$ and $\tilde{V}_{2}$ to denote the effective eg-tt and ge-tt couplings, also with the CT contributions. The authors used the time-dependent wavepacket diffusion method to simulate the SF dynamics with the signs of

the three couplings $\tilde{V}_{1,2}$ and $\tilde{J}$ being modified. It was found that when the product $\tilde{J}_{1} \tilde{V}_{2}$ has a negative (positive) sign, the SF efficiency is enhanced (suppressed). All three couplings, as well as the two $\mathrm{CT}$ energies, are sensitive to inter-chromophore conformations. It is necessary to consider all five factors in tuning packing morphology to enhance SF efficiency.

\subsection{Summary of Theoretical SF Time Scales}

Theoretical SF time scales $\left(\tau_{S F} \mathrm{~s}\right)$ obtained in the vibronic quantum dynamics simulations introduced above are summarized in Table 2 and compared with available experimental results. Pentacene is certainly the most intensely investigated chromophore. A range of its theoretical $\tau_{S F} \mathrm{~s}$ were obtained using a variety of simulation methods. Most of them are in 
good agreement with experimental values. The agreement demonstrates the robustness of the simulation methods in describing SF dynamics. The theory-experiment agreements of the other chromophores are also promising, except for $\mathbf{4 7}$ and $\mathbf{5 2}$ to $\mathbf{5 4}$. The latter three are theoretical designs, for which experimental $\tau_{S F} \mathrm{~s}$ are unavailable. The four theoretical $\tau_{S F} \mathrm{~s}$ of 47 are for different PDI derivatives. They differ significantly in packing geometry and hence their theoretical $\tau_{S F}$ s range from $0.01 \mathrm{ps}$ to $1.8 \mathrm{~ns}$. They shall not be directly compared with the listed experimental $\tau_{S F}$, which is for another PDI derivative. The $\tau_{S F}$ s of the PDI derivatives demonstrate the significant impact of packing geometry on SF dynamics.

Table 2. Simulated SF time scales $\left(\tau_{S F}\right)$ in comparison with experimental values.

\begin{tabular}{|c|c|c|c|c|c|}
\hline Chromophore & Theor. $\tau_{S F}$ & Exp. $\tau_{S F}$ & Chromophore & Theor. $\tau_{S F}$ & Exp. $\tau_{S F}$ \\
\hline \multirow[t]{10}{*}{ Pentacene $\mathbf{3}$} & $0.27 \mathrm{ps}^{139}$ & $0.08 \sim 0.2 \mathrm{ps}^{60,168,216,217}$ & DPB 4 & $3 \mathrm{ps}^{196}$ & $2 \mathrm{ps}^{80}$ \\
\hline & $0.1 \mathrm{ps}^{168}$ & & DPT $\mathbf{2 5}^{a}$ & $1 \mathrm{ps}, 100 \mathrm{ps}^{187}$ & $1 \mathrm{ps}, 100 \mathrm{ps}^{53}$ \\
\hline & $\sim 0.7 \mathrm{ps}^{191}$ & & TIPS-pentacene $e^{b}$ & $0.83 \mathrm{ps}^{191}$ & $\sim 0.1 \mathrm{ps}^{183}$ \\
\hline & $0.06 \sim 0.24 \mathrm{ps}^{192}$ & & & $0.1 \mathrm{ps}^{41}$ & \multirow{3}{*}{$180 \mathrm{ps}^{63}$} \\
\hline & $0.3 \mathrm{ps}^{196}$ & & \multirow{2}{*}{$\mathrm{PDI} \mathbf{4} \mathbf{7}^{c}$} & $0.01 \mathrm{ps}, 1.81 \mathrm{ps}$ & \\
\hline & $0.3 \mathrm{ps}^{201}$ & & & $18, \mathrm{ps}, 1.8 \mathrm{~ns}^{196}$ & \\
\hline & $0.19 \mathrm{ps}^{210}$ & & QOT2 $\mathbf{4 8}^{d}$ & $0.076 \mathrm{ps}^{203}$ & \multirow[t]{3}{*}{$0.064 \mathrm{ps}^{204}$} \\
\hline & $0.1 \sim 0.2 \mathrm{ps}^{211}$ & & 52 & $1 \mathrm{ps}^{207}$ & \\
\hline & $0.2 \mathrm{ps}^{214}$ & & 53 & $12 \mathrm{ps}^{208}$ & \\
\hline & $0.06 \sim 0.09 \mathrm{ps}^{215}$ & & 54 & $3 \mathrm{ps}^{208}$ & \\
\hline
\end{tabular}

${ }^{a}$ The 1 ps corresponds to fast SF at hot spots and the $100 \mathrm{ps}$ is the time scale of exciton diffusion to the hot spots. ${ }^{b}$ The monomer in 31. ${ }^{c}$ The four theoretical $\tau_{S F}$ s are for four PDI derivatives with different packing geometries. The experimental $\tau_{S F}$ is for another derivative. ${ }^{d}$ The $\tau_{S F}$ is for the conversion to the lowest ${ }^{1} A_{g}$ excited state that contains $75 \%$ tt character.

\section{SEPARATION AND SPIN-DISENTANGLEMENT OF THE TRIPLET-PAIR}

Most of the studies introduced above took the singlet-coupled triplet-pair state residing on adjacent chromophores as the SF product state. Relatively fewer works have been dedicated 
to study the evolution of the triplet-pair. The Bardeen group endeavours to investigate the triplet-pair dynamics by applying a magnetic field onto SF systems and examining the delayed fluorescence, which arises from the triplet-triplet fusion. Their studies were summarized in a recent perspective article. ${ }^{34}$ They developed a kinetic model to describe the triplet-pair's evolution, which is demonstrated in Figure $17 . k_{\text {rad }}$ is the radiative decay rate of SE, $k_{\mp 2}\left|C_{s}^{l}\right|^{2}$ the forward and backward rates for SE-to-ME conversion, $k_{\mp 1}$ rates for the triplet to dissociate away and recombine to be adjacent, $k_{\text {relax }}$ the spin-lattice relaxation rate between the nine triplet-pair states, and $k_{\text {trip }}$ the $T_{1}$-to- $S_{0}$ conversion rate. The simulated SE population dynamics is in good agreement with the measured delayed fluorescence dynamics. The relevance of the processes in the model is evident.

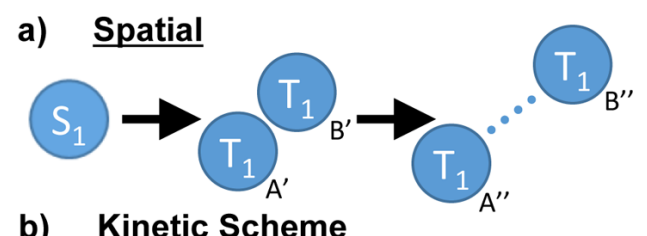

b) Kinetic Scheme

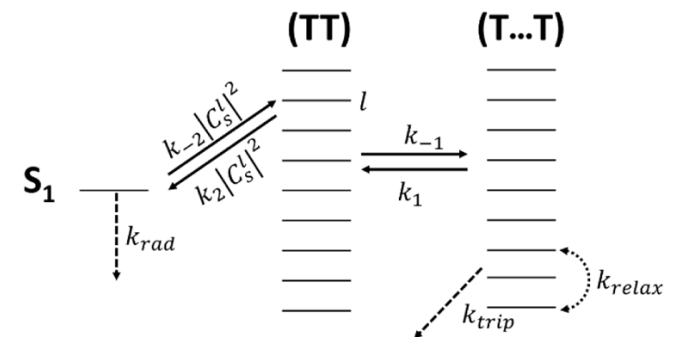

Figure 17. The SF kinetic model developed by Bardeen and coworkers: (a) Illustration of the excitonic states in SF; (b) the processes included in the model. This figure is taken with permission from Ref. 34; Copyright 2014, American Chemical Society.

Of particular importance are the spatial dissociation and the spin relaxation steps in the model. They convert the singlet-coupled triplet-pair to independent triplets. These steps were elucidated by Scholes in $2015 .{ }^{35}$ Scholes pointed out that the two adjacent triplets with an overall singlet spin are bound by mixing with the CT states as shown in Figure 18. The couplings are driven by the transfer integrals shown in the figure. The spatial separation of the triplet-pair is driven by the triplet migration to the next nearest chromophore. Low-lying $\mathrm{CT}$ states are only available for adjacent chromophores; non-adjacent triplets are not bound. Other than the need to overcome the triplet-triplet binding, the spatial dissociation is not much different from triplet hopping. This hopping is a spin-conserved process. Therefore, 
the resultant nonadjacent triplet-pair is still an overall singlet state. Spatial separation does not automatically lead to spin-disentanglement.

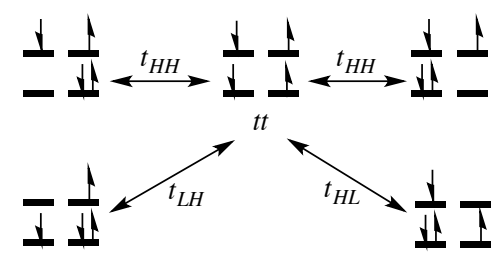

Figure 18. Couplings between $t t$ and CT configurations. The transfer integrals that lead to the couplings are given beside the arrows.

Scholes also elaborated on the difference between triplet-pair separation and triplet-triplet recombination. The spin function of the singlet-coupled triplet-pair reads

$$
{ }^{1}(T T)=\frac{1}{\sqrt{3}}\left(T_{1} T_{-1}-T_{0} T_{0}+T_{-1} T_{1}\right),
$$

where the subscript denotes the $M_{S}$ magnetic quantum number. We label the spinindependent Hamiltonian matrix element for the spatial dissociation $V_{\text {diss }}$. Given two independent triplets, both with $M_{S}=0$, and having migrated to adjacent chromophores, the overlap of their spin function $T_{0} T_{0}$ and ${ }^{1}(T T)$ is $-\frac{1}{\sqrt{3}}$. Consequently, the Hamiltonian matrix element for the triplets to recombine and form the singlet-coupled triplet-pair $V_{r c}=-\frac{1}{\sqrt{3}} V_{d i s s}$. Since a rate constant is proportional to the square of the Hamiltonian matrix element, the recombination rate $k_{r c}\left(k_{1}\right.$ in Figure 17$)$ is $1 / 3$ of that of the dissociation $k_{\text {diss }}\left(k_{-1}\right)$. This relation also applies to two independent triplets with $M_{S}= \pm 1$ and $\mp 1$. The $k_{\text {diss }}>k_{r c}$ relation makes the triplet-triplet separation a favorable process, which facilitates the triplet diffusion to the interface with acceptor and undergo charge separation. In the course of its further spatial separation, the singlet-coupled triplet-pair becomes degenerate in energy with the triplet- and quintet-coupled pairs. These states are then coupled by nuclear-electron and electron-electron spin interactions. These couplings eventually disentangle the two triplets to be independent. This decoherence process is entropy-favored, as it converts 1 singlet-coupled triplet-pair state to 9 independent triplet-pair states.

\section{CONCLUSIONS AND OUTLOOK}

Singlet fission provides an avenue to enhance photoelectric conversion efficiency in organic photovoltaic devices and has attracted plenty of research interest in the past decade. A 
plethora of theoretical studies have been dedicated to this complicated subject. To have thermodynamically favorable singlet fission, a chromophore shall satisfy $E\left(T_{2}\right), E\left(S_{1}\right) \geq$ $2 E\left(T_{1}\right)$. Fundamental electronic structure studies clearly concluded that these requirements are met by molecules with non-negligible yet not too substantial diradical character and negligible tetraradical character. This is the beacon that guides the searches for singlet fission chromophores. New chromophores have been designed or identified based on these guidelines, for instance 1,3-diphenylisobenzofuran and non-polycyclic thienoquinoid compounds. More designs have been proposed and await synthesis. The introduction of donors and acceptors into an aromatic pristine structure to increase its diradical character appeared to be a promising way to design chromophores, especially those of small size. Such captodative effects can be realized by azaborine substitution. More crosslinks between the two vibrant fields of singlet fission and azaborine chemistry can be foreseen in the near future.

Most of the electronic structure studies indicate the dominance a charge-transfermediated mechanism for singlet fission. The large one-electron transfer integrals enter the mediated couplings between single- (SE) and multi-excitonic (ME) states through charge transfer $(\mathrm{CT})$ states, while the small two-electron integrals enter the direct SE-ME couplings. Although the mediated couplings are divided by a CT-SE (or -ME) energy gap, they are still more sizeable than the direct couplings. The two low-lying CT states between adjacent chromophores contribute to the mediated couplings in a destructively interfering way. Alleviating the interference is essential to raise the fission efficiency. The transfer integrals, the CT-SE(ME) gaps, and hence the interference are highly sensitive to inter-chromophore conformation. Engineering the conformation thus plays a central role to improve singlet fission performance. Some recent studies showed that the direct couplings may interfere with the CT-mediated couplings.

Singlet fission is intrinsically a dynamical process with SE-to-ME conversion. Early dynamics simulations were based on electronic Hamiltonians, with imaginary terms that account for dissipation and decoherence phenomenologically. The dissipation and decoherence were more realistically treated by including vibronic couplings in later developed dynamics models. These models include system-bath interaction, with the system being the electronic diabatic states that participate in singlet fission and the bath being a bunch or a continuum of harmonic oscillators. The vibronic couplings are necessary to describe the fission. The Holstein couplings dissipate the energy released in the fission and lead to the SE-ME deco- 
herence, eliminating the Rabi oscillation. Only when the Holstein couplings are included we can have a unidirectional SE-to-ME conversion and an estimate of the fission time scale. The Peierls couplings induce fluctuations of the SE-ME couplings. When the SE-ME couplings are zero due to symmetry, the fluctuations reenable the fission. In more recent dynamics models, vibrational motions were not just treated as a bath. Important vibrational motions that can induce coherent singlet fission were incorporated into the system, giving a more accurate description of the process.

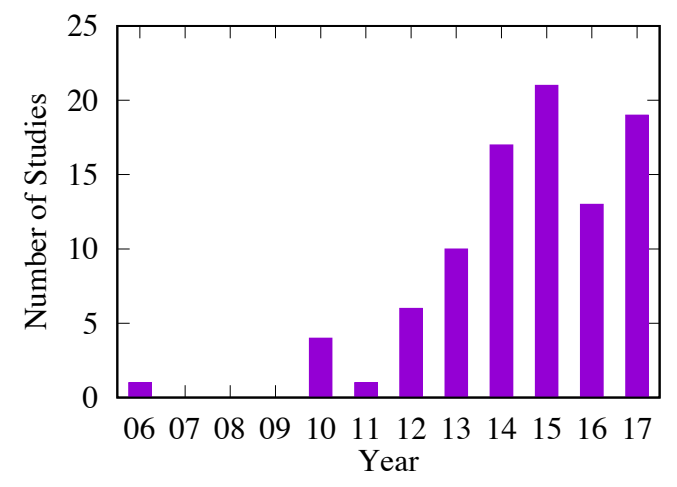

Figure 19. Statistics of publication years for the theoretical studies on singlet fission introduced in this paper.

Figure 19 shows the growth of the theoretical studies on singlet fission that are covered in this review. Theoretical research in this field has been thriving since 2012. It is foreseeable that more relevant studies will be published in the near future, especially in the following directions. (1) Singlet fission models for extended systems that satisfy periodic boundary conditions have been developed. At this moment, the extended models mostly employ parameters obtained from calculations for dimers and small oligomers, which are renormalized to include the effects of solid environment. It is desirable to carry out ab initio calculations and diabatizations for solids. Feasibility of this research direction is determined by the development of excited state calculations for solids. (2) More studies are expected to be dedicated to intramolecular singlet fission (iSF) due to its recent rapid advance. Solvent effects are important for iSF as they can substantially modify the CT energies; they have been considered in some iSF studies at the level of polarizable continuum model. It is desirable to include explicit solvent molecules, especially those with permanent dipole moments, and investigate their impacts on iSF. (3) More theoretical studies are needed to unravel the spatial separation and spin disentanglement of the triplet-pair. Ab initio calculations considering 
electron-electron and electron-nuclei spin interactions are needed to provide parameters for the relevant spin-dependent Hamiltonian. It is also desirable to investigate the couplings of these spin interactions to nuclear vibrations. The spin-vibration couplings eventually disentangle the triplet-pair. (4) The iSF-generated triplet-pair may not be able to dissociate. The pair is likely to reside on the same molecule until it is in contact with an acceptor molecule and undergo charge separation. The difference between harvesting a single triplet and a pair of bound triplets is largely unknown. Studies in this direction are desirable. Given the rapid advances in electronic structure and quantum dynamics methodologies, we are optimistic about the developments in these research directions.

\section{ACKNOWLEDGEMENTS}

We thank Carleton University for the start-up grant (186853) and the Natural Sciences and Engineering Research Council (NSERC) of Canada for funding (RGPIN-2016-06276). 
1 M. B. Smith, J. Michl, Chem. Rev. 2010, 110, 6891-6936.

2 M. B. Smith, J. Michl, Annu. Rev. Phys. Chem. 2013, 64, 361-386.

3 V. K. Thorsmølle, R. D. Averitt, J. Demsar, D. L. Smith, S. Tretiak, R. L. Martin, X. Chi, B. K. Crone, A. P. Ramirez, A. J. Taylor, Phys. Rev. Lett. 2009, 102, 017401.

4 M. W. B. Wilson, A. Rao, J. Clark, R. S. S. Kumar, D. Brida, G. Cerullo, R. H. Friend, J. Am. Chem. Soc. 2011, 133, 11830-11833.

5 H. Marciniak, M. Fiebig, M. Huth, S. Schiefer, B. Nickel, F. Selmaier, S. Lochbrunner, Phys. Rev. Lett. 2007, 99, 176402.

6 W.-L. Chan, M. Ligges, A. Jailaubekov, L. Kaake, L. Miaja-Avila, X.-Y. Zhu, Science 2011, 334, 1541-1545.

7 W.-L. Chan, M. Ligges, X.-Y. Zhu, Nature Chem. 2012, 4, 840-845.

8 M. J. Bearpark, F. Bernardi, S. Clifford, M. Olivucci, M. A. Robb, B. R. Smith, T. Vreven, J. Am. Chem. Soc. 1996, 118, 169-175.

9 W. Shockley, H. J. Queisser, J. Appl. Phys. 1961, 32, 510-519.

10 M. C. Hanna, A. J. Nozik, J. Appl. Phys. 2006, 100, 074510.

11 J. Lee, P. Jadhav, M. A. Baldo, Appl. Phys. Lett. 2009, 95, 033301.

12 D. N. Congreve, J. Lee, N. J. Thompson, E. Hontz, S. R. Yost, P. D. Reusswig, M. E. Bahlke, S. Reineke, T. Van Voorhis, M. A. Baldo, Science 2013, 340, 334-337.

13 N. J. Thompson, D. N. Congreve, D. Goldberg, V. M. Menon, M. A. Baldo, Appl. Phys. Lett. 2013, 103, 263302.

14 L. M. Pazos-Outón, J. M. Lee, M. H. Futscher, A. Kirch, M. Tabachnyk, R. H. Friend, B. Ehrler, ACS Energy Lett. 2017, 2, 476-480.

15 S. N. Sanders, E. Kumarasamy, A. B. Pun, K. L. Steigerwald, M. Y. Sfeir, L. M. Campos, Chem 2016, 1, 505-511.

16 J. Xia, S. N. Sanders, W. Cheng, J. Z. Low, J. Liu, L. M. Campos, T. Sun, Adv. Mater. 2017, 29, 1601652 .

17 J. Lee, P. Jadhav, P. D. Reusswig, S. R. Yost, N. J. Thompson, D. N. Congreve, E. Hontz, T. Van Voorhis, M. A. Baldo, Acc. Chem. Res. 2013, 46, 1300-1311.

18 H. Köppel, W. Domcke, L. S. Cederbaum, Adv. Chem. Phys. 1984, 59, 57-246. 
19 K. Bhattacharyya, A. Datta, J. Phys. Chem. C 2017, 121, 1412-1420.

20 T. C. Berkelbach, M. S. Hybertsen, D. R. Reichman, J. Chem. Phys. 2013, 138, 114102.

21 P. J. Vallett, J. L. Snyder, N. H. Damrauer, J. Phys. Chem. A 2013, 117, 10824-10838.

22 R. A. Marcus, Annu. Rev. Phys. Chem. 1964, 15, 155-196.

23 J. C. Johnson, A. J. Nozik, J. Michl, Acc. Chem. Res. 2013, 46, 1290-1299.

24 N. Monahan, X.-Y. Zhu, Annu. Rev. Phys. Chem. 2015, 66, 601-618.

25 J. Z. Low, S. N. Sanders, L. M. Campos, Chem. Mater. 2015, 27, 5453-5463.

26 E. Busby, J. Xia, Q. Wu, J. Z. Low, R. Song, J. R. Miller, X.-Y. Zhu, L. M. Campos, M. Y. Sfeir, Nature Mater. 2015, 14, 426-433.

27 M. T. Trinh, Y. Zhong, Q. Shen, T. Schiros, S. Jockusch, M. Y. Sfeir, M. Steigerwald, C. Nuckolls, X.-Y. Zhu, J. Phys. Chem. C 2015, 119, 1312-1319.

28 J. Zirzlmeier, D. Lehnherr, P. B. Coto, E. T. Chernick, R. Casillas, B. S. Basel, M. Thoss, R. R. Tykwinski, D. M. Guldi, Proc. Natl. Acad. Sci. USA 2015, 112, 5325-5330.

29 S. N. Sanders, E. Kumarasamy, A. B. Pun, K. Appavoo, M. L. Steigerwald, L. M. Campos, M. Y. Sfeir, J. Am. Chem. Soc. 2016, 138, 7289-7297.

30 S. N. Sanders, E. Kumarasamy, A. B. Pun, M. T. Trinh, B. Choi, J. Xia, E. J. Taffet, J. Z. Low, J. R. Miller, X. Roy, X.-Y. Zhu, M. L. Steigerwald, M. Y. Sfeir, L. M. Campos, J. Am. Chem. Soc. 2015, 137, 8965-8972.

31 S. Lukman, A. J. Musser, K. Chen, S. Athanasopoulos, C. K. Yong, Z. Zeng, Q. Ye, C. Chi, J. M. Hodgkiss, J. Wu, R. H. Friend, N. C. Greenham, Adv. Funct. Mater. 2015, 25, 5452-5461. 32 N. V. Korovina, S. Das, Z. Nett, X. Feng, J. Joy, R. Haiges, A. I. Krylov, S. E. Bradforth, M. E. Thompson, J. Am. Chem. Soc. 2016, 138, 617-627.

33 J. Zirzlmeier, R. Casillas, S. R. Reddy, P. B. Coto, D. Lehnherr, E. T. Chernick, I. Papadopoulos, M. Thoss, R. R. Tykwinski, D. M. Guldi, Nanoscale 2016, 8, 10113-10123.

34 G. B. Piland, J. J. Burdett, R. J. Dillon, C. J. Bardeen, J. Phys. Chem. Lett. 2014, 5, 23122319 .

35 G. D. Scholes, J. Phys. Chem. A 2015, 119, 12699-12705.

36 M. J. Y. Yayebjee, S. N. Sanders, E. Kumarasamy, L. M. Campos, M. Y. Sfeir, D. R. McCamey, Nature Phys. 2017, 13, 182-188.

37 B. S. Basel, J. Zirzlmeier, C. Hetzer, B. T. Phelan, M. D. Krzyaniak, S. R. Reddy, P. B. Coto, N. E. Horwitz, R. M. Young, F. J. White, F. Hampel, T. Clark, M. Thoss, R. R. Tykwinski, 
M. R. Wasielewski, D. M. Guldi, Nature Commun. 2017, 8, 15171.

38 R. Tempelaar, D. R. Reichman, J. Chem. Phys. 2017, 146, 174703.

39 A. B. Kolomeisky, X. Feng, A. I. Krylov, J. Phys. Chem. C 2014, 118, 5188-5195.

40 R. E. Peierls, Quantum Theory of Solids., Clarendon Press, Oxford, UK, 1996.

41 H. Tamura, M. Huix-Rotllant, I. Burghardt, Y. Olivier, D. Beljonne, Phys. Rev. Lett. 2015, 115, 107401.

42 E. C. Alguire, J. E. Subotnik, N. H. Damrauer, J. Phys. Chem. A 2015, 119, 299-311.

43 S. Singh, W. J. Jones, W. Siebrand, B. P. Stoicheff, W. G. Schneider, J. Chem. Phys. 1965, 42, 330-342.

44 C. E. Swenberg, W. T. Stacy, Chem. Phys. Lett. 1968, 2, 327-328.

45 N. E. Geacintov, M. Pope, F. Vogel, Phys. Rev. Lett. 1969, 22, 593-596.

46 R. P. Groff, P. Avakian, R. E. Merrifield, Phys. Rev. B 1970, 1, 815-817.

47 C. E. Swenberg, R. van Metter, M. Ratner, Chem. Phys. Lett. 1972, 16, 482-485.

48 J. Burgos, M. Pope, C. E. Swenberg, R. R. Alfano, Phys. Status Solid. B 1977, 83, 249-256.

49 Y. Tomkiewicz, R. P. Groff, P. Avakian, J. Chem. Phys. 1971, 54, 4504-4507.

50 J. J. Burdett, A. M. Müller, D. Gosztola, C. J. Bardeen, J. Chem. Phys. 2010, 133, 144506.

51 J. J. Burdett, D. Gosztola, C. J. Bardeen, J. Chem. Phys. 2011, 135, 214508.

52 J. J. Burdett, C. J. Bardeen, J. Am. Chem. Soc. 2012, 134, 8597-8607.

53 S. T. Robert, R. E. McAnally, J. N. Mastron, D. H. Webber, M. T. Whited, R. L. Brutchey, M. E. Thompson, S. E. Bradforth, J. Am. Chem. Soc. 2012, 134, 6388-6400.

54 J. N. Mastron, S. T. Robert, R. E. McAnally, M. E. Thompson, S. E. Bradforth, J. Phys. Chem. B 2013, 117, 15519-15526.

55 H. Najafov, B. Lee, Q. Zhou, L. C. Feldman, V. Podzorov, Nature Mater. 2010, 9, 938-943.

56 A. Ryasnyanskiy, I. Biaggio, Phys. Rev. B 2011, 84, 193203.

57 L. Ma, K. Zhang, C. Kloc, H. Sun, M. E. Michel-Beyerle, G. G. Gurzadyan, Phys. Chem. Chem. Phys. 2012, 14, 8307-8312.

58 V. Jankus, E. W. Snedden, D. W. Bright, E. Arac, D. Dai, A. P. Monkman, Phys. Rev. B 2013, 87, 224202.

59 A. Rao, M. W. B. Wilson, J. M. Hodgkiss, S. Albert-Seifried, H. Bässler, R. H. Friend, J. Am. Chem. Soc. 2010, 132, 12698-12703. 
60 A. Rao, M. W. B. Wilson, S. Albert-Seifried, R. Di Pietro, R. H. Friend, Phys. Rev. B 2011, 84, 195411.

61 C. Ramanan, A. L. Smeigh, J. E. Anthony, T. J. Marks, M. R. Wasielewski, J. Am. Chem. Soc. 2012, 134, 386-397.

62 B. J. Walker, A. J. Musser, D. Beljonne, R. H. Friend, Nature Chem. 2013, 5, 1019-1024.

63 S. W. Eaton, L. E. Shoer, S. D. Karlen, S. M. Dyar, E. A. Margulies, B. S. Veldkamp, C. Ramanan, D. A. Hartzler, S. Savikhin, T. J. Marks, M. R. Wasielewski, J. Am. Chem. Soc. 2013, $135,14701-14712$.

64 B. Kraabel, D. Hulin, C. Aslangul, C. Lapersonne-Meyer, M. Schott, Chem. Phys. 1998, 22\%, $83-98$.

65 C. C. Gradinaru, J. T. M. Kennis, E. Papagiannakis, I. H. M. van Stokkum, R. J. Cogdell, G. R. Fleming, R. A. Niederman, R. van Grondelle, Proc. Natl. Acad. Sci. USA 2001, 98, $2364-2369$.

66 C. Wang, D. E. Schlamadinger, V. Desai, M. J. Tauber, ChemPhysChem 2011, 12, 2891-2894.

67 C. Wang, M. J. Tauber, J. Am. Chem. Soc. 2010, 132, 13988-13991.

68 A. J. Musser, M. Al-Hashimi, M. Maiuri, D. Brida, M. Heeney, G. Cerullo, R. H. Friend, J. Clark, J. Am. Chem. Soc. 2013, 135, 12747-12754.

69 S. Kawata, Y.-J. Pu, A. Saito, Y. Kurashige, T. Beppu, H. Katagiri, M. Hada, J. Kido, Adv. Mater. 2016, 28, 1585-1590.

70 M. Kasha, Discuss Faraday Soc. 1950, 9, 14-19.

71 S. K. Lower, M. A. El-Sayed, Chem. Rev. 1966, 66, 199-241.

72 M. A. El-Sayed, Acc. Chem. Res. 1968, 1, 8-16.

73 I. Paci, J. C. Johnson, X. D. Chen, G. Rana, D. Popović, D. E. David, A. J. Nozik, M. A. Ratner, J. Michl, J. Am. Chem. Soc. 2006, 128, 16546-16553.

74 M. Bendikov, H. M. Duong, K. Starkey, K. N. Houk, E. A. Carter, F. Wudl, J. Am. Chem. Soc. 2004, 126, 7416-7417.

75 J. Hachmann, J. J. Dorando, M. Avilés, G. K.-L. Chan, J. Chem. Phys. 2007, 127, 134309.

76 Y. Yang, E. R. Davidson, W. Yang, Proc. Natl. Acad. Sci. USA 2016, 113, E5098-E5107.

77 R. Pariser, R. G. Parr, J. Chem. Phys. 1953, 21, 466-471.

78 R. Pariser, R. G. Parr, J. Chem. Phys. 1953, 21, 767-776.

79 J. A. Pople, Trans. Faraday Soc. 1953, 49, 1375-1385. 
80 J. C. Johnson, A. Nozik, J. Michl, J. Am. Chem. Soc. 2010, 132, 16302-16303.

81 J. L. Ryerson, J. N. Schrauben, A. J. Ferguson, S. C. Sahoo, P. Naumov, Z. Havlas, J. Michl, A. J. Nozik, J. C. Johnson, J. Phys. Chem. C 2014, 118, 12121-12132.

82 J. N. Schrauben, J. L. Ryerson, J. Michl, J. C. Johnson, J. Am. Chem. Soc. 2014, 136, 7363-7373.

83 A. F. Schwerin, J. C. Johnson, M. B. Smith, P. Sreearunothai, D. Popović, J. Černý, Z. Havlas, I. Paci, A. Akdag, M. K. MacLeod, X. Chen, D. E. David, M. A. Ratner, J. R. Miller, A. J. Nozik, J. Michl, J. Phys. Chem. A 2010, 114, 1457-1473.

84 J. C. Johnson, J. Michl, Top. Curr. Chem. 2017, 375, 80.

85 T. Minami, M. Nakano, J. Phys. Chem. Lett. 2012, 3, 145-150.

86 T. Minami, S. Ito, M. Nakano, J. Phys. Chem. Lett. 2012, 3, 2719-2723.

87 M. Kertesz, C. H. Choi, S. Yang, Chem. Rev. 2005, 105, 3448-3481.

88 S. Motomura, M. Nakano, H. Fukui, K. Yoneda, T. Kubo, R. Carion, B. Champagne, Phys. Chem. Chem. Phys. 2011, 13, 20575-20583.

89 S. Ito, T. Minami, M. Nakano, J. Phys. Chem. C 2012, 116, 19729-19736.

90 T. Minami, S. Ito, M. Nakano, J. Phys. Chem. Lett. 2013, 4, 2133-2137.

91 K. Kamada, K. Ohta, A. Shimizu, T. Kubo, R. Kishi, H. Takahashi, E. Botek, B. Champagne, M. Nakano, J. Phys. Chem. Lett. 2010, 1, 973-940.

92 S. Ito, M. Nakano, J. Phys. Chem. C 2015, 119, 148-157.

93 S. Ito, T. Nagami, M. Nakano, J. Phys. Chem. Lett. 2016, 7, 3925-3930.

94 A. Akdag, Z. Havlas, J. Michl, J. Am. Chem. Soc. 2012, 134, 14624-14631.

95 H. G. Viehe, Z. Janousek, R. Merenyi, L. Stella, Acc. Chem. Res. 1985, 18, 148-154.

96 T. Zeng, N. Ananth, R. Hoffmann, J. Am. Chem. Soc. 2014, 136, 12638-12647.

97 P. G. Campbell, A. J. V. Marwitz, S.-Y. Liu, Angew. Chem. Int. Ed. 2012, 51, 6074-6092.

98 J. Wen, Z. Havlas, J. Michl, J. Am. Chem. Soc. 2015, 137, 165-172.

99 K. Bhattacharyya, S. M. Pratik, A. Datta, J. Phys. Chem. C 2015, 119, 25696-25702.

100 Z. Liang, W. Zhao, S. Wang, Q. Tang, S. C. Lam, Q. Miao, Org. Lett. 2008, 10, 2007-2010.

101 M. Yamada, I. Ikemoto, H. Kuroda, Bull. Chem. Soc. Jpn. 1988, 61, 1057-1062.

102 Y. Chen, L. Shen, X. Li, J. Phys. Chem. A 2014, 118, 5700-5708.

103 A. Maliakal, K. Raghavachari, H. Katz, E. Chandross, T. Siegrist, Chem. Mater. 2004, 16, 4980-4986. 
104 I. Kaur, W. Jia, R. P. Kopreski, S. Selvarasah, M. R. Dokmeci, C. Pramanik, N. E. McGruer, G. P. Miller, J. Am. Chem. Soc. 2008, 130, 16274-16286.

105 S. S. Palayangoda, R. Mondal, B. K. Shah, D. C. Neckers, J. Org. Chem. 2007, 72, 6584-6587.

106 S. Li, Y. Chen, X. Li, C. Li, J. Mol. Graphics Mod. 2016, 66, 187-195.

107 P. Würfel, Physics of Solar Cells: From Basic Principles to Advanced Concepts, WILEY-VCH Verlag, Weinheim, 2009.

108 R. Grotjahn, T. M. Maier, J. Michl, M. Kaupp, J. Chem. Theory Comput. 2017, 13, 4984-4996.

109 E. C. Greyson, B. R. Stepp, X. Chen, A. F. Schwerin, I. Paci, M. B. Smith, A. Akdag, J. C. Johnson, A. J. Nozik, J. Michl, M. A. Ratner, J. Phys. Chem. B 2010, 114, 14223-14232.

110 T. S. Kuhlman, J. Kongsted, K. V. Mikkelsen, K. B. Møller, T. I. Sølling, J. Am. Chem. Soc. 2010, 132, 3431-3439.

111 P. M. Zimmerman, F. Bell, D. Casanova, M. Head-Gordon, J. Am. Chem. Soc. 2011, 133, 19944-19952.

112 P. M. Zimmerman, Z. Zhang, C. B. Musgrave, Nature Chem. 2010, 2, 648-652.

113 T. Zeng, R. Hoffmann, N. Ananth, J. Am. Chem. Soc. 2014, 136, 5755-5764.

114 Y.-K. Choe, H. A. Witek, J. P. Finley, K. Hirao, J. Chem. Phys. 2001, 114, 3913-3918.

115 H. A. Witek, Y.-K. Choe, J. P. Finley, K. Hirao, J. Comput. Chem. 2002, 23, 957-965.

116 P. B. Coto, S. Sharifzadeh, J. B. Neaton, M. Thoss, J. Chem. Theory Comput. 2015, 11, $147-156$.

117 C. Zener, Proc. R. Soc. Lond. A 1932, 137, 696-702.

118 C. Zener, Proc. R. Soc. Lond. A 1933, 140, 660-668.

119 P. M. Zimmerman, C. B. Musgrave, M. Head-Gordon, Acc. Chem. Res. 2013, 46, 1339-1347.

120 D. Beljonne, H. Yamagata, J. L. Brédas, F. C. Spano, Y. Olivier, Phys. Rev. Lett. 2013, 110, 226402.

121 H. Yamagata, J. Norton, E. Hontz, Y. Olivier, D. Beljonne, J. L. Brédas, R. J. Silbey, F. C. Spano, J. Chem. Phys. 2011, 134, 204703.

122 D. Casanova, J. Chem. Theory Comput. 2014, 10, 324-334.

123 D. Casanova, J. Chem. Theory Comput. 2015, 11, 2642-2650.

124 S. Sharifzadeh, P. Darancet, L. Kronik, J. B. Neaton, J. Phys. Chem. Lett. 2013, 4, 2197-2201.

125 Y. Wu, K. Liu, H. Liu, Y. Zhang, H. Zhang, J. Yao, H. Fu, J. Phys. Chem. Lett. 2014, 5, 3451-3455. 
126 S. R. Yost, J. Lee, M. W. B. Wilson, T. Wu, D. P. McMahon, R. R. Parkhurst, N. J. Thompson, D. N. Congreve, A. Rao, K. Johnson, M. Y. Sfeir, M. Bawendi, T. M. Swager, R. H. Friend, M. A. Baldo, T. Van Voorhis, Nature Chem. 2014, 6, 492-497.

127 J. Jortner, M. Bixon, J. Chem. Phys. 1988, 88, 167-170.

128 E. Busby, T. C. Berkelbach, B. Kumar, A. Chernikov, Y. Zhong, H. Hlaing, X.-Y. Zhu, T. F. Heinz, M. S. Hybertsen, M. Y. Sfeir, D. R. Reichman, C. Nuckolls, O. Yaffe, J. Am. Chem. Soc. 2014, 136, 10654-10660.

129 C.-H. Yang, C.-P. Hsu, J. Phys. Chem. Lett. 2015, 6, 1925-1929.

130 S. F. Boys, Rev. Mod. Phys. 1960, 32, 296-299.

131 J. M. Foster, S. F. Boys, Rev. Mod. Phys. 1960, 32, 300-302.

132 E. S. Medvedev, A. A. Stuchebrukhov, J. Chem. Phys. 1997, 10\%, 3821-3831.

133 K. Miyata, Y. Kurashige, K. Watanabe, T. Sugimoto, S. Takahashi, S. Tanaka, J. Takeya, T. Yanai, Y. Matsumoto, Nature Chem. 2017, 9, 983-989.

134 N. H. Damrauer, J. L. Snyder, J. Phys. Chem. Lett. 2015, 6, 4456-4462.

135 E. G. Fuemmeler, S. N. Sanders, A. B. Pun, E. Kumarasamy, T. Zeng, K. Miyata, M. L. Steigerwald, X.-Y. Zhu, M. Y. Sfeir, L. M. Campos, N. Ananth, ACS Cent. Sci. 2016, 2, $316-324$.

136 R. W. A. Havenith, H. D. de Gier, R. Broer, Mol. Phys. 2012, 110, 2445-2454.

137 M. Wibowo, R. Broer, R. W. A. Havenith, Comput. Theor. Chem. 2017, 1116, 190-194.

138 X. Feng, A. V. Luzanov, A. I. Krylov, J. Phys. Chem. Lett. 2013, 4, 3845-3852.

139 T. C. Berkelbach, M. S. Hybertsen, D. R. Reichman, J. Chem. Phys. 2014, 141, 074705.

140 L. S. Cederbaum in Conical intersections: electronic structure, dynamics and spectroscopy, W. Domcke, D. R. Yarkony, H. Köppel (Eds.), World Scientific, 2004, chapter 1, pp. 3-40.

141 B. K. Kendrick, C. A. Mead, D. G. Truhlar, Chem. Phys. 2002, 277, 31-41.

142 S. Matsika, X. Feng, A. V. Luzanov, A. I. Krylov, J. Phys. Chem. A 2014, 118, 11943-11955.

143 X. Feng, A. B. Kolomeisky, A. I. Krylov, J. Phys. Chem. C 2014, 118, 19608-19617.

144 X. Feng, A. I. Krylov, Phys. Chem. Chem. Phys. 2016, 18, 7751-7761.

145 J. J. Burdett, C. J. Bardeen, Acc. Chem. Res. 2013, 46, 1312-1320.

146 X. Feng, D. Casanova, A. I. Krylov, J. Phys. Chem. C 2016, 120, 19070-19077.

147 K. Aryanpour, A. Shukla, S. Mazumdar, J. Phys. Chem. C 2015, 119, 6966-6979. 
148 A. M. Müller, Y. S. Avlasevich, W. W. Schoeller, K. Müllen, C. J. Bardeen, J. Am. Chem. Soc. 2007, 129, 14240-14250.

149 A. M. Müller, Y. S. Avlasevich, K. Müllen, C. J. Bardeen, Chem. Phys. Lett. 2006, 421, $518-522$.

150 K. Aryanpour, T. Dutta, U. N. V. Huynh, Z. V. Vardeny, S. Mazumdar, Phys. Rev. Lett. 2015, 115, 267401.

151 D. Fazzi, G. Grancini, M. Maiuri, D. Brida, G. Cerullo, G. Lanzani, Phys. Chem. Chem. Phys. 2012, 14, 6367-6374.

152 G. Grancini, M. Maiuri, D. Fazzi, A. Petrozza, H.-J. Egelhaaf, D. Brida, G. Cerullo, G. Lanzani, Nature Mater. 2013, 12, 29-33.

153 J. Ren, Q. Peng, X. Zhang, Y. Yi, Z. Shuai, J. Phys. Chem. Lett. 2017, 8, 2175-2181.

154 P. Petelenz, M. Snamina, G. Mazur, J. Phys. Chem. C 2015, 119, 14338-14342.

155 E. V. Tsiper, Z. G. Soos, Phys. Rev. B 2003, 68, 085301.

156 P. Petelenz, M. Snamina, J. Phys. Chem. C 2015, 119, 28570-28576.

157 P. Petelenz, M. Snamina, J. Phys. Chem. Lett. 2016, 7, 1913-1916.

158 M. Snamina, P. Petelenz, ChemPhysChem 2017, 18, 149-155.

159 S. M. Parker, T. Seideman, M. A. Ratner, T. Shiozaki, J. Phys. Chem. C 2014, 118, 1270012705.

160 S. Ito, T. Nagami, M. Nakano, J. Phys. Chem. Lett. 2015, 6, 4972-4977.

161 T. Sato, K. Tokunaga, K. Tanaka, J. Chem. Phys. 2006, 124, 024314.

162 P. C. P. de Andrade, J. A. Freire, J. Chem. Phys. 2004, 120, 7811-7819.

163 S. Ito, T. Nagami, M. Nakano, J. Phys. Chem. A 2016, 120, 6236-6241.

164 C. A. Coulson, B. O’Leary, R. B. Mallion, Hückel Theory for Organic Chemists, Academic Press, Inc., London, 1978.

165 S. Ito, T. Nagami, M. Nakano, RSC Adv. 2017, 7, 34830-34845.

166 T. Nagami, S. Ito, T. Kubo, M. Nakano, ACS Omega 2017, 2, 5095-5103.

167 S. Ito, T. Nagami, M. Nakano, Phys. Chem. Chem. Phys. 2017, 19, 5737-5745.

168 W.-L. Chan, T. C. Berkelbach, M. R. Provorse, N. R. Monahan, J. Tritsch, M. S. Hybertsen, D. R. Reichman, J. Gao, X.-Y. Zhu, Acc. Chem. Res. 2013, 46, 1321-1329.

169 N. J. Mayhall, J. Chem. Theory Comput. 2016, 12, 4263-4273.

170 V. Abraham, N. J. Mayhall, J. Phys. Chem. Lett. 2017, 8, 5472-5478. 
171 F. Ambrosio, A. Troisi, J. Chem. Phys. 2014, 141, 204703.

172 P. E. Teichen, J. D. Eaves, J. Chem. Phys. 2015, $143,044118$.

173 E. A. Buchanan, Z. Havlas, J. Michl in Adv. Quantum Chem., Vol. 75, J. R. Sabin, E. J. Br andas (Eds.), Elsevier, 2017, chapter 7, pp. 175-227.

174 M. Wolfsberg, L. Helmholz, J. Chem. Phys. 1952, 20, 837-843.

175 R. Hoffmann, J. Chem. Phys. 1963, 39, 1397-1412.

176 E. A. Buchanan, J. Michl, J. Am. Chem. Soc. 2017, 139, 15572-15575.

177 Z. Havlas, J. Michl, Isr. J. Chem. 2015, 56, 96-106.

178 E. C. Greyson, J. Vura-Weis, J. Michl, M. A. Ratner, J. Phys. Chem. B 2010, 114, 1416814177.

179 H. C. Longuet-Higgins, M. de. V. Roberts, Proc. R. Soc. Lond. A 1954, 224, 336-347.

180 P. E. Teichen, J. D. Eaves, J. Phys. Chem. B 2012, 116, 11473-11481.

181 T. C. Berkelbach, M. S. Hybertsen, D. R. Reichman, J. Chem. Phys. 2013, 138, 114103.

182 M. L. Tiago, J. E. Northrup, S. G. Louie, Phys. Rev. B 2003, 67, 115212.

183 A. J. Musser, M. Liebel, C. Schnedermann, T. Wende, T. B. Kehoe, A. Rao, P. Kukura, Nature Phys. 2015, 11, 352-357.

184 A. A. Bakulin, S. E. Morgan, T. B. Kehoe, M. W. B. Wilson, A. W. Chin, D. Zigmantas, D. Egorova, A. Rao, Nature Chem. 2016, 8, 16-23.

185 N. R. Monahan, D. Sun, H. Tamura, K. W. Williams, B. Xu, Y. Zhong, B. Kumar, C. Nuckolls, A. R. Harutyunyan, G. Chen, H.-L. Dai, D. Beljonne, X.-Y. Zhu, Nature Chem. 2017, 9, 341346.

186 R. Tempelaar, D. R. Reichman, J. Chem. Phys. 2017, 146, 174704.

187 W. Mou, S. Hattori, P. Rajak, F. Shimojo, A. Nakano, Appl. Phys. Lett. 2013, 102, 173301.

188 J. C. Tully, J. Chem. Phys. 1990, 93, 1061-1071.

189 A. V. Akimov, O. V. Prezhdo, J. Am. Chem. Soc. 2014, 136, 1599-1608.

190 L. J. Wang, O. V. Prezhdo, J. Phys. Chem. Lett. 2014, 5, 713-719.

191 L. Wang, Y. Olivier, O. V. Prezhdo, D. Beljonne, J. Phys. Chem. Lett. 2014, 5, 3345-3353.

192 G. Tao, J. Phys. Chem. C 2014, 118, 17299-17305.

193 G. Tao, J. Phys. Chem. C 2014, 118, 27258-27264.

194 G. Tao, J. Chem. Theory Comput. 2015, 11, 28-36.

195 N. Renaud, P. A. Sherratt, M. A. Ratner, J. Phys. Chem. Lett. 2013, 4, 1065-1069. 
196 F. Mirjani, N. Renaud, N. Gorczak, F. C. Grozema, J. Phys. Chem. C 2014, 118, 14192-14199.

197 N. Renaud, F. C. Grozema, J. Phys. Chem. Lett. 2015, 6, 360-365.

198 J. N. Schrauben, Y. Zhao, C. Mercado, P. I. Dron, J. L. Ryerson, J. Michl, K. Zhu, J. C. Johnson, ACS Appl. Mater. Interfaces 2015, 7, 2286-2293.

199 S. E. Strong, J. D. Eaves, J. Phys. Chem. Lett. 2015, 6, 1209-1215.

200 M. W. B. Wilson, A. Rao, K. Johnson, S. Gélinas, R. Di Pietro, J. Clark, R. H. Friend, J. Am. Chem. Soc. 2013, 135, 16680-16688.

201 J. Zheng, Y. Xie, S. Jiang, Z. Lan, J. Phys. Chem. C 2016, 120, 1375-1389.

202 O. Varnavski, N. Abeyasinghe, J. Aragó, J. J. Serrano-Pérez, E. Ortí, J. T. López Navarrete, K. Takimiya, D. Casanova, J. Casado, T. Goodson, J. Phys. Chem. Lett. 2015, 6, 1375-1384. 203 A. D. Chien, A. R. Molina, N. Abeyasinghe, O. P. Varnavski, T. Goodson, P. M. Zimmerman, J. Phys. Chem. C 2015, 119, 28258-28268.

204 Z. Wang, T. Kobayashi, New. J. Phys. 2008, 10, 123021-123034.

205 L. Ren, F. Liu, X. Shen, C. Zhang, Y. Yi, X. Zhu, J. Am. Chem. Soc. 2015, $13 \%$.

206 M. R. Momenti, J. Chem. Theory Comput. 2016, 12, 5067-5075.

207 T. Zeng, P. Goel, J. Phys. Chem. Lett. 2016, 7, 1351-1358.

208 T. Zeng, J. Phys. Chem. Lett. 2016, 7, 4405-4412.

209 S. Prodhan, S. Ramasesha, Phys. Rev. B 2017, 96, 075142.

210 M. Nakano, S. Ito, T. Nagami, Y. Kitagawa, T. Kubo, J. Phys. Chem. C 2016, 120, 2280322815.

211 Y. Fujihashi, A. Ishizaki, J. Phys. Chem. Lett. 2016, 7, 363-369.

212 A. F. Morrison, J. M. Herbert, J. Phys. Chem. Lett. 2017, 8, 1442-1448.

213 Z. Huang, Y. Fujihashi, Y. Zhao, J. Phys. Chem. Lett. 2017, 8, 3306-3312.

214 M. A. Castellanos, P. Huo, J. Phys. Chem. Lett. 2017, 8, 2480-2488.

215 H. Zang, Y. Zhao, W. Liang, J. Phys. Chem. Lett. 2017, 8, 5105-5112.

216 M. W. B. Wilson, A. Rao, B. Ehrler, R. H. Friend, Acc. Chem. Res. 2013, 46, 1330-1338.

217 C. Jundt, G. Klein, B. Sipp, J. Le Moigne, M. Joucla, A. A. Villaeys, Chem. Phys. Lett. 1995, 241, 84-88. 